Florida International University FIU Digital Commons

$11-14-2014$

\title{
Development of a Surface-Enhanced Raman Spectroscopy Method for the Detection of Benzodiazepines in Urine
}

Erika L. Doctor

Florida International University, erika.doctor@outlook.com

DOI: $10.25148 /$ etd.FI14110743

Follow this and additional works at: https:// digitalcommons.fiu.edu/etd

Part of the Analytical Chemistry Commons, and the Other Chemistry Commons

\section{Recommended Citation}

Doctor, Erika L., "Development of a Surface-Enhanced Raman Spectroscopy Method for the Detection of Benzodiazepines in Urine" (2014). FIU Electronic Theses and Dissertations. 1642.

https://digitalcommons.fiu.edu/etd/1642 


\title{
FLORIDA INTERNATIONAL UNIVERSITY
}

Miami, Florida

\section{DEVELOPMENT OF A SURFACE-ENHANCED RAMAN SPECTROSCOPY METHOD FOR THE DETECTION OF BENZODIAZEPINES IN URINE}

A dissertation submitted in partial fulfillment of

\author{
the requirements for the degree of \\ DOCTOR OF PHILOSOPHY \\ in \\ CHEMISTRY \\ by
}

Erika L. Doctor

2014 
To: Interim Dean Michael R. Heithaus

College of Arts and Sciences

This dissertation, written by Erika L. Doctor, and entitled Development of a SurfaceEnhanced Raman Spectroscopy Method for the Detection of Benzodiazepines in Urine, having been approved in respect to style and intellectual content, is referred to you for judgment.

We have read this dissertation and recommend that it be approved.

$\begin{array}{r}\hline \text { Jose Almirall } \\ \hline \text { Jaroslava Miksovska } \\ \hline \text { Fenfei Leng } \\ \hline \text { Chenzhong Li } \\ \hline \text { Bruce McCord, Major Professor }\end{array}$

Date of Defense: November 14, 2014

The dissertation of Erika L. Doctor is approved.

Interim Dean Michael R. Heithaus College of Arts and Sciences

Dean Lakshmi N. Reddi University Graduate School

Florida International University, 2014 
(C) Copyright 2014 by Erika L. Doctor All rights reserved. 


\section{DEDICATION}

This thesis is dedicated to all of my grandparents. I know you all would have been so proud of me, Pépère, you always joked about me becoming a doctor, well now I'll be Doctor $^{2}$. Granny, I'm such a strong woman because of you and I could not have gotten through all this without that. Mémère, while I was one of the last, you were still there for me until you could not remember. 


\section{ACKNOWLEDGMENTS}

I would like to acknowledge Florida International University for their support for the six years I have been in the program. I would also like to thank my committee, Jose Almirall, Jaroslava Miksovska, Fenfei Leng, and Chenzhong Li, for their input and knowledge over the course of this project and making the time for all the meetings.

Bruce McCord, my major professor, thank you for all the insight and always having faith in this work even when my faith waivered. I will never forget when you told me to skip class and go on a honeymoon, you always provided me with the right balance of work and adventure which helped get me through this entire process. Anthony McGoron at FIU made this work possible through the use of his instrumentation.

To my fellow doctors and doctors in training, a huge thank you for pretty much everything. Many of us went through, or are going through a lot of the same trials and tribulations and we have always been there to support and push each other to make it through. All the hugs, you can do its, answers on where and when paperwork is due, spontaneous trips to Orlando, baseball games, and tutu tutorials made this journey worth it. Your friendships mean more to me than I can express in words and I can't wait to see what the future holds.

To all my other friends, those close and far away, thank you for understanding the struggle (even if you had no idea what I was talking about) and being there for me. My life would not be the same without all of you. Megan, even though you are so far away, you took every phone call and text message whether I was distraught or elated. I knew we would be partners in crime since the day you asked me to be your roommate. Without 
your support and commiserating through all of this I would have given up on myself years ago.

Finally I want to thank my family, Mom \& Dad, you have seen my highs and lows throughout my life and have always supported every decision I've ever made, including this one. Mom, you are my best friend and I thank you for always being there to talk me down for the ledge. Dad, don't ask any questions. To The Doctor (the original), thank you for dealing with my crazy, all my yelling and crying would have scared anyone else away but not you. Maybe it's because you have two hearts that you have been able to deal with me over the past eight years. I love you and I cannot imagine my life without you. 


\title{
ABSTRACT OF THE DISSERTATION \\ DEVELOPMENT OF A SURFACE-ENHANCED RAMAN SPECTROSCOPY METHOD FOR THE DETECTION OF BENZODIAZEPINES IN URINE
}

\author{
by \\ Erika L. Doctor \\ Florida International University, 2014 \\ Miami, Florida \\ Professor Bruce McCord, Major Professor
}

Benzodiazepines are among the most prescribed compounds for anti-anxiety and are present in many toxicological screens. These drugs are also prominent in the commission of drug facilitated sexual assaults due their effects on the central nervous system. Due to their potency, a low dose of these compounds is often administered to victims; therefore, the target detection limit for these compounds in biological samples is $10 \mathrm{ng} / \mathrm{mL}$. Currently these compounds are predominantly analyzed using immunoassay techniques; however more specific screening methods are needed.

The goal of this dissertation was to develop a rapid, specific screening technique for benzodiazepines in urine samples utilizing surface-enhanced Raman spectroscopy (SERS), which has previously been shown be capable of to detect trace quantities of pharmaceutical compounds in aqueous solutions. Surface enhanced Raman spectroscopy has the advantage of overcoming the low sensitivity and fluorescence effects seen with conventional Raman spectroscopy. The spectra are obtained by applying an analyte onto a SERS-active metal substrate such as colloidal metal particles. SERS signals can be 
further increased with the addition of aggregate solutions. These agents cause the nanoparticles to amass and form hot-spots which increase the signal intensity.

In this work, the colloidal particles are spherical gold nanoparticles in aqueous solution with an average size of approximately $30 \mathrm{~nm}$. The optimum aggregating agent for the detection of benzodiazepines was determined to be $16.7 \mathrm{mM} \mathrm{MgCl}_{2}$, providing the highest signal intensities at the lowest drug concentrations with limits of detection between 0.5 and $127 \mathrm{ng} / \mathrm{mL}$. A supported liquid extraction technique was utilized as a rapid clean extraction for benzodiazepines from urine at a $\mathrm{pH}$ of 5.0, allowing for clean extraction with limits of detection between 6 and $640 \mathrm{ng} / \mathrm{mL}$. It was shown that at this $\mathrm{pH}$ other drugs that are prevalent in urine samples can be removed providing the selective detection of the benzodiazepine of interest.

This technique has been shown to provide rapid (less than twenty minutes), sensitive, and specific detection of benzodiazepines at low concentrations in urine. It provides the forensic community with a sensitive and specific screening technique for the detection of benzodiazepines in drug facilitated assault cases. 


\section{TABLE OF CONTENTS}

CHAPTER

PAGE

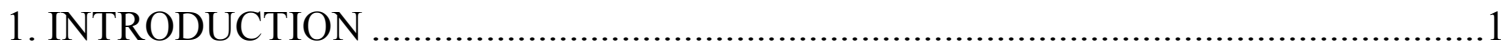

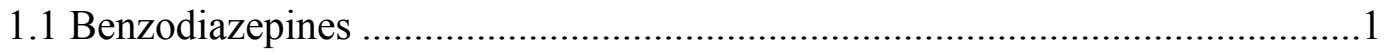

1.1.1 Development and structure ………….............................................

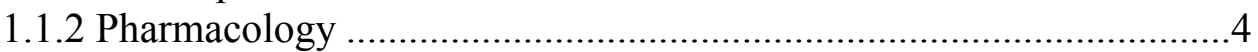

1.1.3 Prevalence in Drug Facilitated Sexual Assaults ..................................

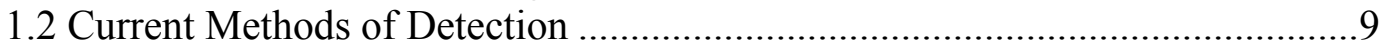

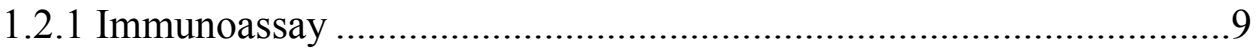

1.2.2 Gas chromatography mass spectrometry ........................................13

1.3 Surface Enhanced Raman Spectroscopy ..........................................................15

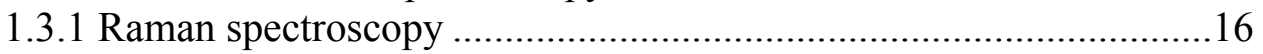

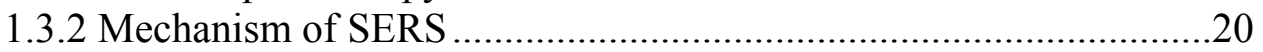

1.3.3 Improvement of enhancement through aggregation ...........................34

1.3.4 Nanoparticle formation and properties ...............................................36

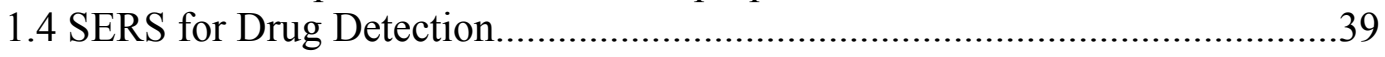

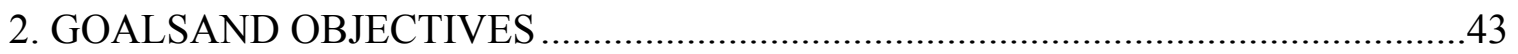

3. DETERMINATIONOF SURFACE ENHANCED SUBSTRATE ……………...........44

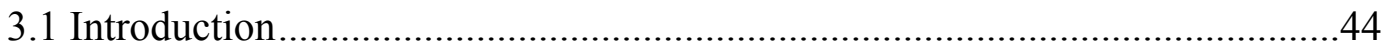

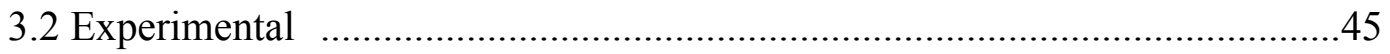

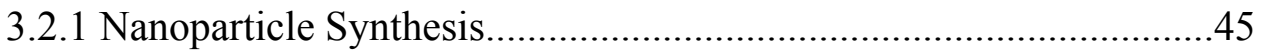

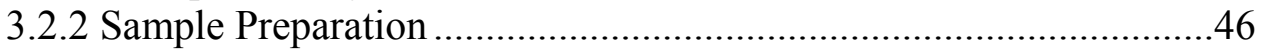

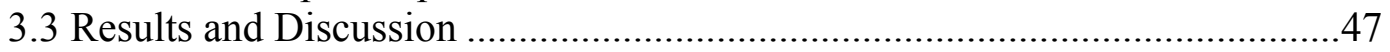

3.3.1 Size characterization of particles ....................................................4

3.3.2 Surface enhanced Raman validation...............................................50

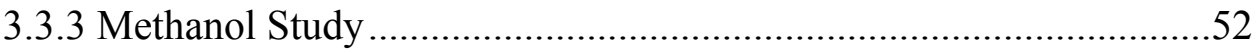

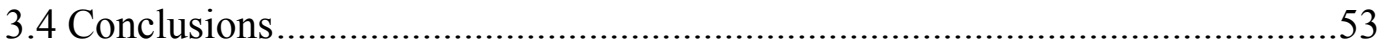

4. COMPARISON OF AGGREGATION AGENTS FOR THE SURFACEENHANCED RAMAN ANALYSIS BENZODIAZPINES ..........................................56

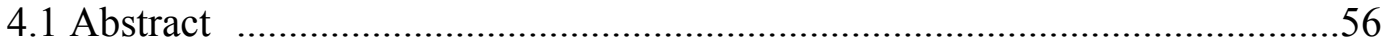

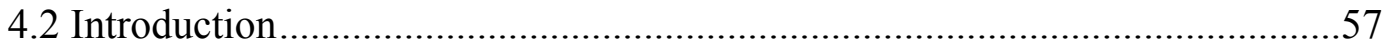

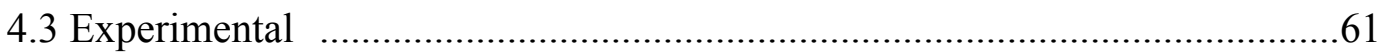

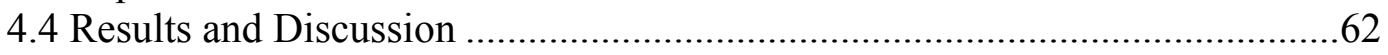

4.4.1 Nanoparticle Characterization ........................................................62

4.4.2 Comparison of Different Aggregating Agents...................................63

4.4.3 $\mathrm{MgCl}_{2}$ Concentration Optimization ..................................................69

4.4.4 Proof of Concept - Spiked Urine Sample...........................................71

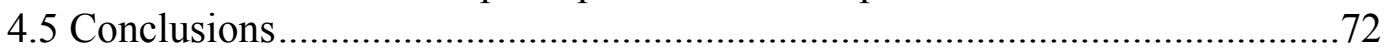

5. SUPPORTEDLIQUID EXCTRATION OF BENZODIAZPINES FROM URINE......74

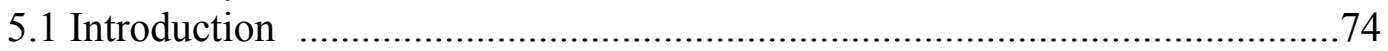




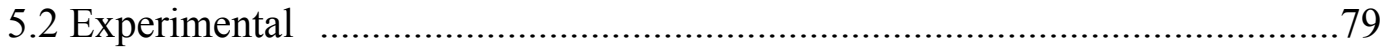

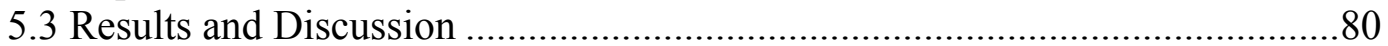

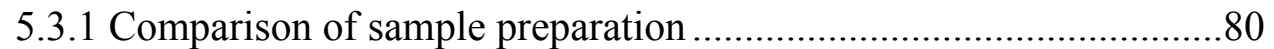

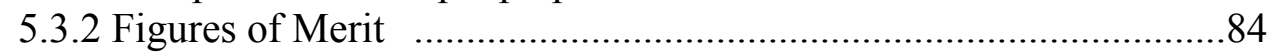

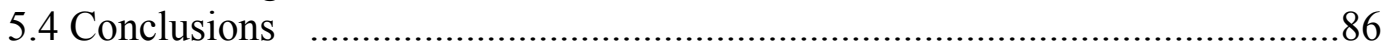

6. INTERFERENCE STUDY OF COMMOMLY ENCOUNTERED DRUGS FOUND IN DRUG FACILITATED SEXUAL ASSAULT SAMPLES .........................................8

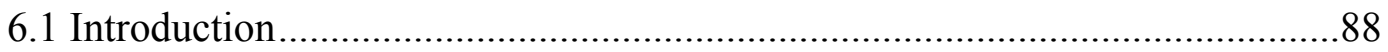

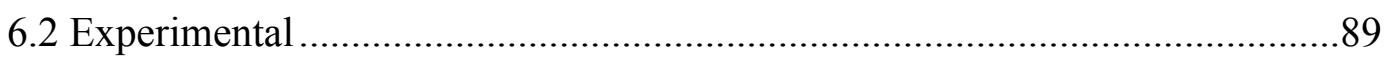

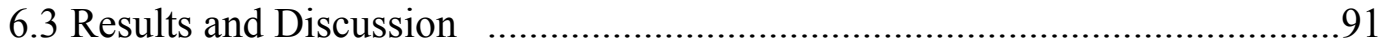

6.3.1 Pure interference compounds..........................................................91

6.3.2 Extraction of 7-amino-flunitrazepam and interference mixtures......94

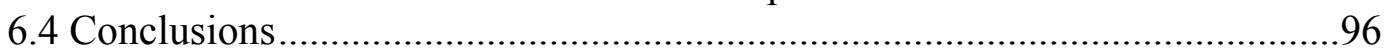

7.VIBRATIONAL SPECTRAL ANALYSIS OF ELEVEN BENZODIAZEPINES .......97

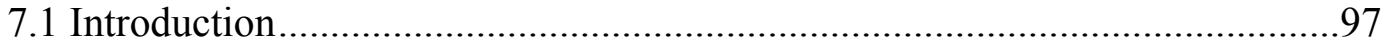

7.2 Analysisof Peaks for All Benzodiazepines and Major Subgroups .................100

7.3 Additional Spectral Analysis of Individual Benzodiazepines .......................105

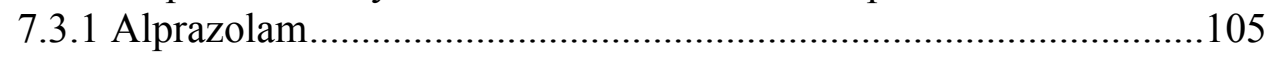

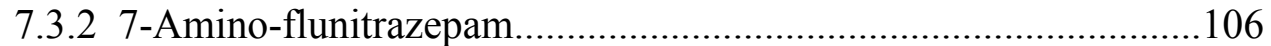

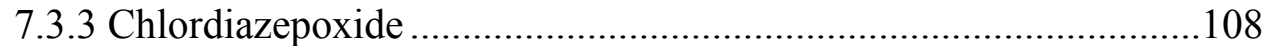

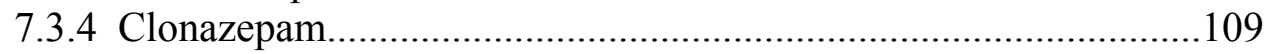

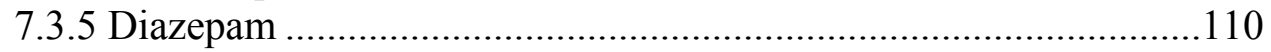

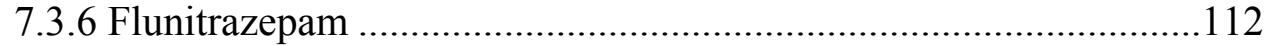

7.3.7 Lorazepam............................................................................113

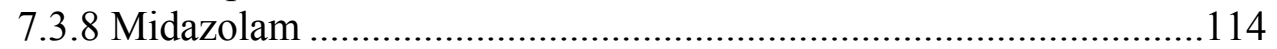

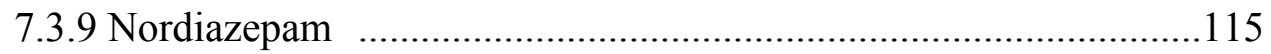

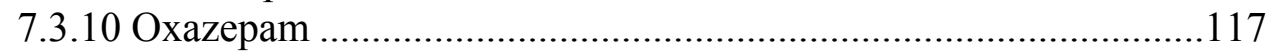

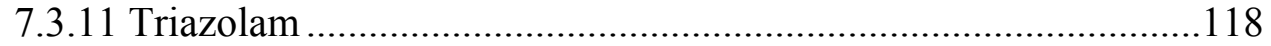

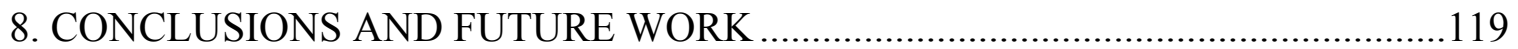

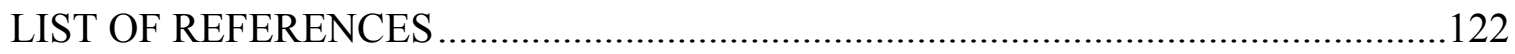

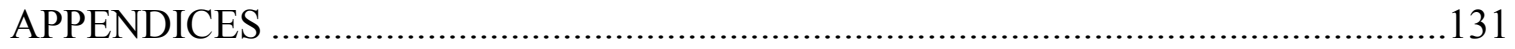

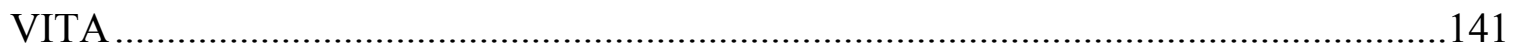




\section{LIST OF TABLES}

TABLE

PAGE

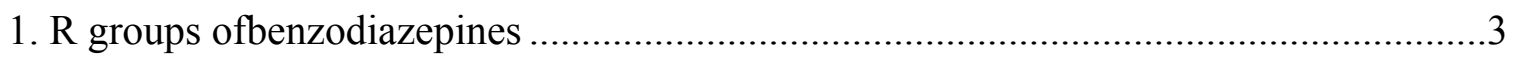

2. R groups for eleven benzodiazepines on basestructures ........................................60

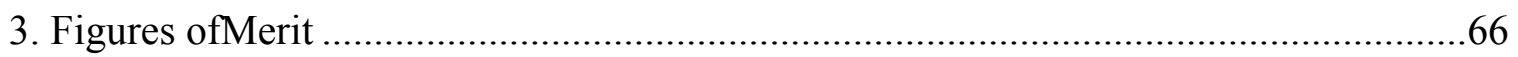

4. $\mathrm{pKa}$ and ionization data on elevenbenzodiazepines............................................... 78

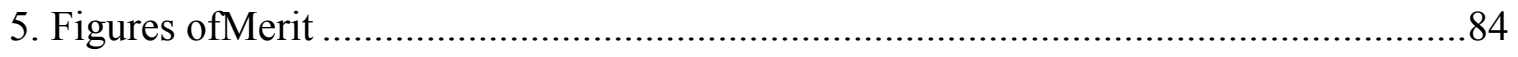

6. Interference compounds studied with common urine concentrations andpKas............89

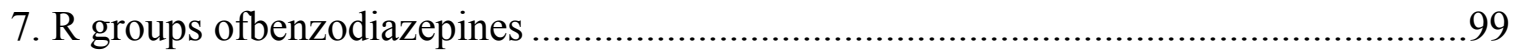

8. Observed wavenumbers $\left(\mathrm{cm}^{-1}\right)$ in the surface enhanced Raman spectra of all benzodiazepines studied.

9. Observed wavenumbers $\left(\mathrm{cm}^{-1}\right)$ in the surface enhanced Raman spectra of 1,2triazolo and imidazo benzodiazepinesstudied.......................................................... 102

10. Observed wavenumbers $\left(\mathrm{cm}^{-1}\right)$ in the surface enhanced Raman spectra of monosubstituted benzene b-ring

11. Observed wavenumbers $\left(\mathrm{cm}^{-1}\right)$ in the surface enhanced Raman spectra of $\mathrm{N}-\mathrm{CH}_{3}$ stretch.

12. Observed wavenumbers $\left(\mathrm{cm}^{-1}\right)$ in the surface enhanced Raman spectra of $C=O$ deformation on diazepinering

13. Observed wavenumbers $\left(\mathrm{cm}^{-1}\right)$ in the surface enhanced Raman spectra of C-Cl stretching.....

14. Observed wavenumbers $\left(\mathrm{cm}^{-1}\right)$ in the surface enhanced Raman Spectra of C-F stretching.....

15. Observed wavenumbers $\left(\mathrm{cm}^{-1}\right)$ in the surface enhanced Raman spectra of alprazolam.

16. Observed wavenumbers $\left(\mathrm{cm}^{-1}\right)$ in the surface enhanced Raman spectra of 7-amino-flunitrazepam. 
17. Observed wavenumbers $\left(\mathrm{cm}^{-1}\right)$ in the surface enhanced Raman spectra of chlordiazepoxide

18. Observed wavenumbers $\left(\mathrm{cm}^{-1}\right)$ in the surface enhanced Raman spectra of clonazepam

19. Observed wavenumbers $\left(\mathrm{cm}^{-1}\right)$ in the surface enhanced Raman spectra of diazepam

20. Observed wavenumbers $\left(\mathrm{cm}^{-1}\right)$ in the surface enhanced Raman spectra of flunitrazepam

21. Observed wavenumbers $\left(\mathrm{cm}^{-1}\right)$ in the surface enhanced Raman spectra of lorazepam

22. Observed wavenumbers $\left(\mathrm{cm}^{-1}\right)$ in the surface enhanced Raman spectra of midazolam

23. Observed wavenumbers $\left(\mathrm{cm}^{-1}\right)$ in the surface enhanced Raman spectra of nordiazepam 116

24. Observed wavenumbers $\left(\mathrm{cm}^{-1}\right)$ in the surface enhanced Raman spectra of oxazepam

25. Observed wavenumbers $\left(\mathrm{cm}^{-1}\right)$ in the surface enhanced Raman spectra of triazolam 


\section{LIST OF FIGURES}

FIGURE

PAGE

1. Distribution of benzodiazepine manufacture in 2011 bytype.......................2

2. Base structures of (A) 1,4-benzodiazepines and (B) 1,2-triazolo- and 1,2-imidazo

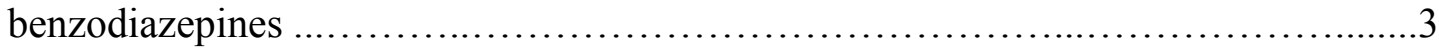

3. $\mathrm{GABA}_{\mathrm{A}}$ receptor without benzodiazepine and withbenzodiazepine................4

4. Routes of metabolism for some 1 , 4 benzodiazepines............................6

5. Routes of metabolism for 1,2-triazolo and 1,2-imidazobenzodiazepines...............7

6. Theory of enzyme multiplied immunoassay technique assay...................... 11

7. Simplified Jablonski diagrams for Rayleigh, Stokes, and anti-Stokesprocesses........17

8. Example Raman spectra................................................. 18

9. The real (a) and imaginary (b) parts of the dielectric function for Ag andAu........22

10. Reflectance at normal incidence for Ag and Au using the dielectric function shown in Figure 8

11. Local field intensity enhancement factor (LFIEF) at the surface of the metal for

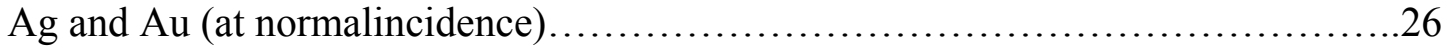

12. Representation of localized surface plasmon resonances of a cylinder ............ 27

13. Local field intensity enhancement factor at point $\mathrm{A}$ on a sphere of $\mathrm{Au}$ or $\mathrm{Ag}$ in the electrostatic approximation......................................... 30

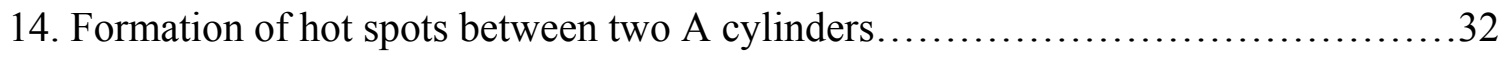

15. UV-visible absorbance and SEM micrograph of AgI particles ..............................47

16. UV-visible absorbance and SEM micrograph of AgIIparticles.....................48

17. UV-visible absorbance and SEM micrograph of AgIII particles.....................49

18. UV-visible absorbance and SEM micrograph of gold particles .............................49 


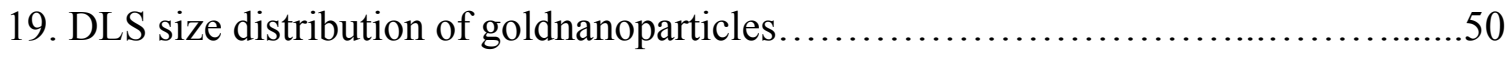

20. SERS comparison of chlordiazepoxide between four synthesizedparticles .................51

21. Comparison of methanol spectra with chlordiazepoxide $\mathrm{HCl}$ in $10 \%$ methanol..........53

22. Base structures of 1,4-benzodiazepines (A) and 1,2-triazolo-benzodiazepines (B).... 60

23. UV-Visible spectra of gold nanoparticles, 30 nmparticles .........................................63

24. SERS spectra at $250 \mathrm{ng} / \mathrm{mL}$ with $\mathrm{MgCl}_{2}$ (1.67M) aggregation; indicated peaks were used for calibrationcurves ..............................................................................65

25. Comparison of $\mathrm{MgCl}_{2}$ and $\mathrm{NaCl}$ aggregation for alprazolam detection......................68

26. Comparison of $\mathrm{MgCl}_{2}$ and $\mathrm{CaCl}_{2}$ aggregation for alprazolam detection.....................69

27. Comparison of 3 concentrations of $\mathrm{MgCl}_{2}$ aggregating reagent for all eleven benzodiazepines at their lowest observableconcentration............................70

28. SERS spectra of 7-amino-flunitrazepam standard compared to SERS spectra of

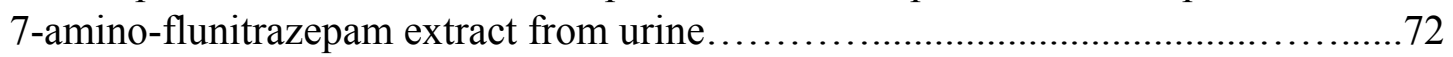

29. Work flow of supported liquidextraction.......................................... 77

30. SERS signals for $250 \mathrm{ng} / \mathrm{mL} 7$-amino-flunitrazepam extracted with three SLE preparation techniques and liquid-liquid extraction

31. SERS spectra of (A) flunitrazepam and (B) 7-amino-flunitrazepam using hydrolysis sample preparation; compared to SERS spectra of pure (C) flunitrazepam

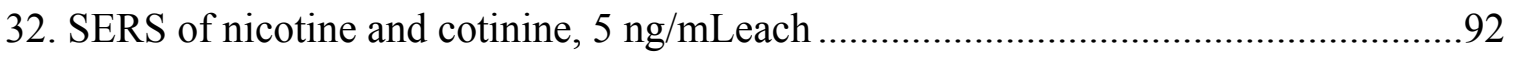

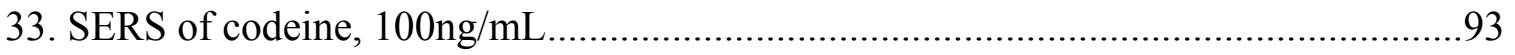

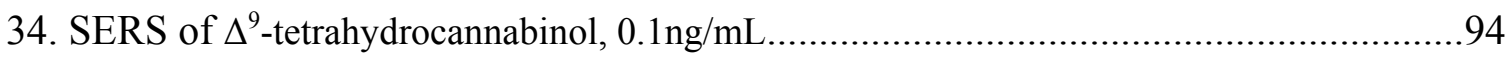

35. SERS spectra of extraction of interference mixtures as compared to the SERS

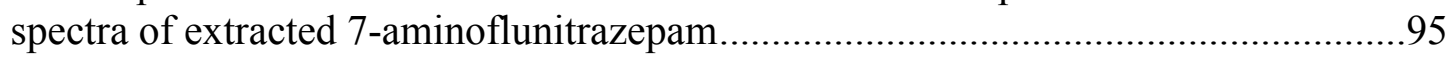

36. Base structures of (A) 1,4-benzodiazepines and (B) 1,2-triazolo- and 1,2-imidazo

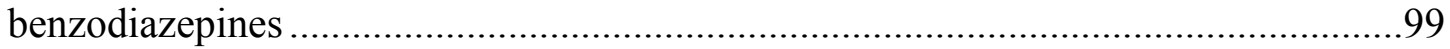

37. SERS spectra alprazolam ..................................................................................106 


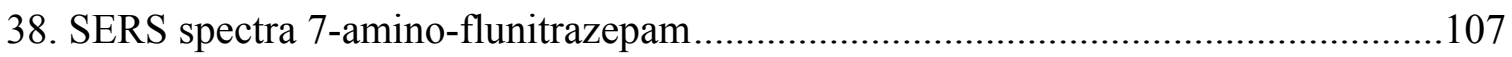

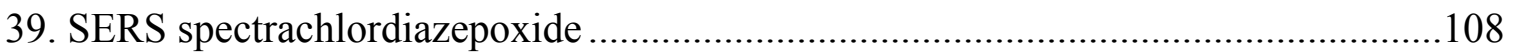

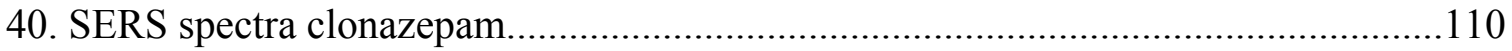

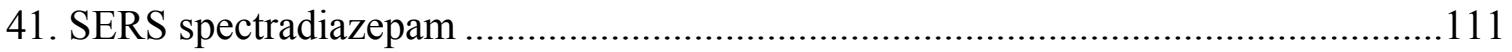

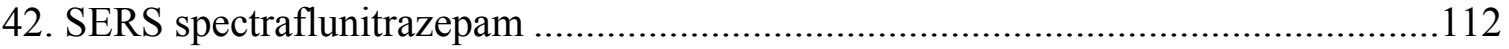

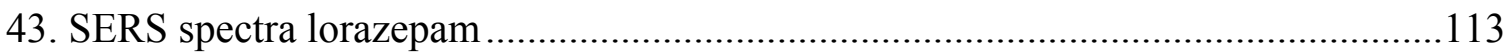

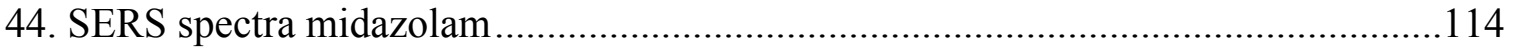

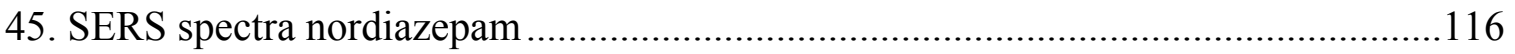

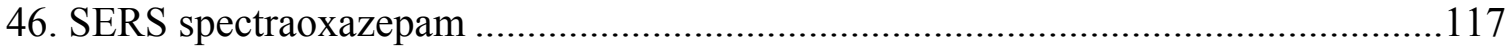

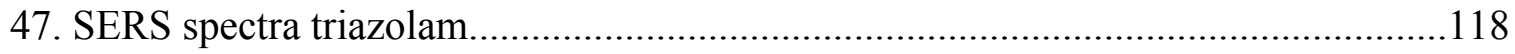

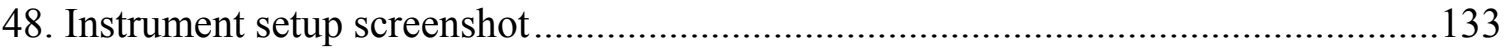

49. Picture of proper configuration with sample holder for cuvette analysis .................134

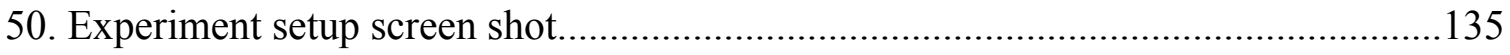

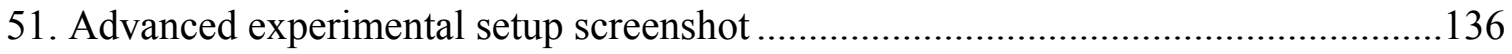

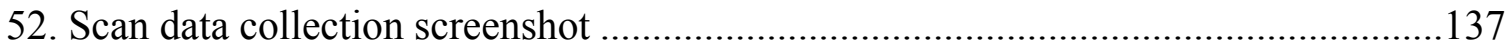


$\Delta \mathrm{A} \quad$ Change in absorbance

Ag Silver

$\mathrm{AgNO}_{3} \quad$ Silver nitrate

$\mathrm{Au} \quad$ Gold

$\mathrm{CaCl}_{2} \quad$ Calcium chloride

CCD Charge coupled device

CE Capillary electrophoresis

$\mathrm{Cl} \quad$ Chloride

CNS Central nervous system

DCM Dichloromethane

DFSA Drug facilitated sexual assault

DLS Dynamic light scattering

DOB 2,5,-dimethoxy-4-bromoamphetamine

E Energy

EF Enhancement factor

GABA Gama-amino butyric acid

GC Gas chromatography

GHB Gamma-hydroxybutyrate

$\mathrm{HAuCl}_{4} \quad$ Chloroauric acid

$\mathrm{HCl} \quad$ Hydrochloric acid

HPLC High performance liquid chromatography

IR Infrared 


\begin{tabular}{|c|c|}
\hline $\mathrm{KCl}$ & Potassium chloride \\
\hline $\mathrm{KNO}_{3}$ & Potassium nitrate \\
\hline LFIEF & local field intensity enhancement factor \\
\hline LLE & Liquid-liquid extraction \\
\hline $\mathrm{m}$ & moderate \\
\hline M & Molarity \\
\hline mg & Milligram \\
\hline $\mathrm{MgCl}_{2}$ & Magnesium chloride \\
\hline $\mathrm{mL}$ & Milliliter \\
\hline MS & Mass spectrometry \\
\hline $\mathrm{NaCl}$ & Sodium chloride \\
\hline NAD & Nicotinamide adeten dinucleotide \\
\hline ng & Nanogram \\
\hline $\mathrm{NH}_{4} \mathrm{OAc}$ & Ammonium acetate \\
\hline $\mathrm{s}$ & strong \\
\hline SEM & Scanning electron microscopy \\
\hline SERS & Surface enhanced Raman spectroscopy \\
\hline SLE & Supported liquid extraction \\
\hline SPE & Solid phase extraction \\
\hline str & stretch \\
\hline THC & $\Delta^{9}$-tetrahydrocannabinol \\
\hline$\mu \mathrm{g}$ & Microgram \\
\hline UV/Vis & Ultra-violet visible spectroscopy \\
\hline
\end{tabular}


Voltage

vibr

vibrations

VS

Very strong

W

weak

xviii 


\section{INTRODUCTION}

Benzodiazepines are commonly prescribed and easily accessible drugs in the United States which are often found during toxicological testing in cases of drug facilitated sexual assault (DFSA). Many of the screening methods that are currently used are lengthy to perform, complicated, or do not successfully detect low concentrations of these types of drugs. The goal of this dissertation was to develop a rapid screening technique for the detection of low concentration benzodiazepines in DFSA cases using surface enhanced Raman spectroscopy (SERS). The use of Raman spectroscopy not only detects the drug, it also permits the determination of spectral information, highly useful in the presumptive identification of unknown compounds.

\subsection{Benzodiazepines}

\subsubsection{Development and structure}

Benzodiazepines were first developed in the 1950s. The discovery of these compounds caused a surge in the development of new compounds that would have superior pharmacological effects over existing tranquilizers, such as barbiturates. The first benzodiazepine, chlordiazepoxide $\left(\right.$ Librium $\left.^{\circledR}\right)$, was unexpectedly synthesized by Sternbach in 1955 as a result of a ring extension of a quinazoline-3- $N$-oxide derivative. ${ }^{1}$ The synthesis of homologues and analogs of this first compound was patented in $1959^{2}$, and since then many structurally similar compounds have been created and marketed by drug companies for therapeutic uses as tranquilizers, hypnotics, and anxiolytics. ${ }^{3}$ As of the 2012 International Narcotic Control Board statistics, there were 35 benzodiazepines under international control, with alprazolam and diazepam being the most manufactured in the world as shown in Figure $1 .{ }^{4}$ 


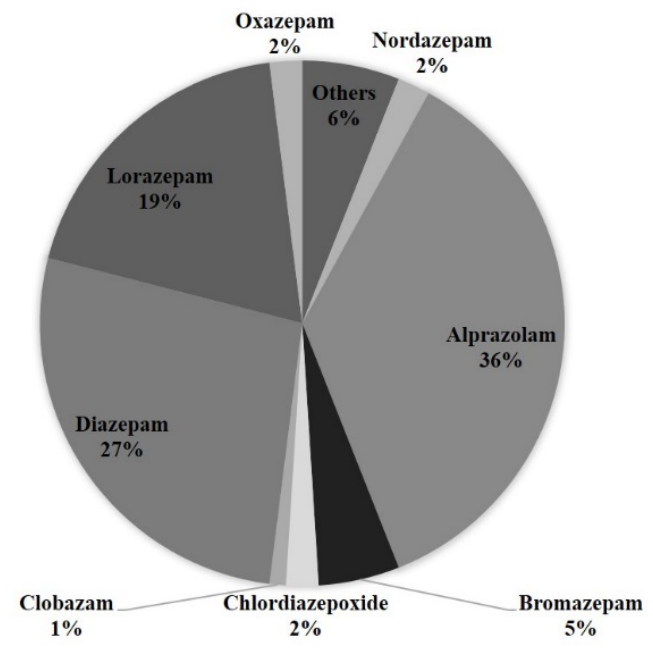

Figure 1: Distribution of benzodiazepine manufacture in 2011 by type ${ }^{4}$

The structure of benzodiazepines are based on a 5-aryl-1,4-benzodiazpine backbone where the 6-7 bond of the 1,4-diazepine ring is fused to a benzene ring. The aryl substituent at the 5 position is usually either a phenyl (diazepam) or 2'-halophenyl (flunitrazepam). The main base structure constitutes the class of 1,4-benzodiazepines. ${ }^{3}$ More recent benzodiazepines, developed in the 1980s, have an additional 1,3-diazole ring or 1,2,4-triazole linked to the 1-2 bond of the 1,4-diazepine ring, forming the class of 1,2imidazo and 1,2-triazolo-benzodiazepines, respectively (Figure 2, Table 1). ${ }^{3}$ The different substituents on the general structures change the duration of action and potency of the drug. For example, the addition of an electron withdrawing group, such as a nitro group or a chlorine, on the benzene ring generally enhances activity. ${ }^{5}$ 

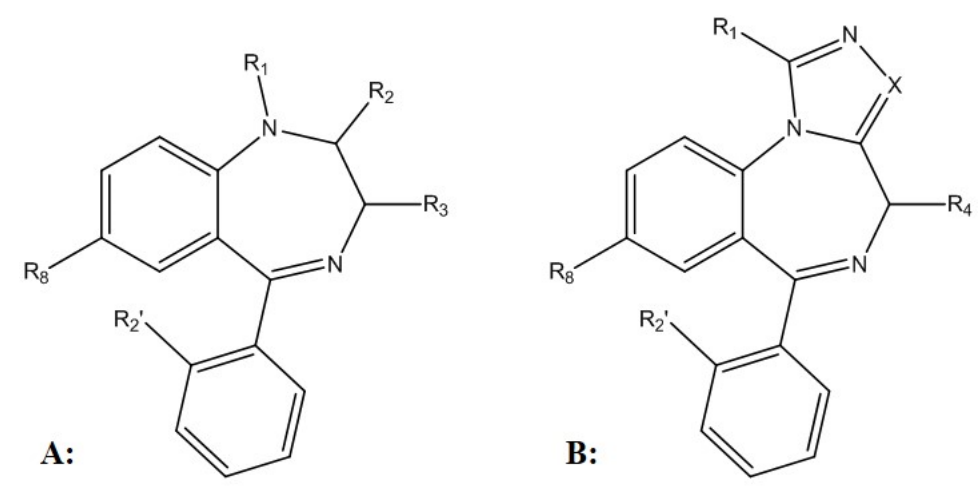

Figure 2: Base structures of (A) 1,4-benzodiazepines and (B) 1,2-triazolo- and 1,2imidazo benzodiazepines ${ }^{6}$

\begin{tabular}{|l|c|c|c|c|c|}
\hline 1,2- Triazolo- and 1,2-imidazo-benzodiazepines & $\mathbf{R}_{\mathbf{1}}$ & $\mathbf{X}$ & $\mathbf{R}_{\mathbf{4}}$ & $\mathbf{R}_{\mathbf{2}}$, & $\mathbf{R}_{\mathbf{8}}$ \\
\hline Alprazolam & $-\mathrm{CH}_{3}$ & $=\mathrm{N}-$ & $-\mathrm{H}$ & $-\mathrm{H}$ & $-\mathrm{Cl}$ \\
\hline Midazolam & $-\mathrm{CH}_{3}$ & $=\mathrm{C}-$ & $-\mathrm{H}$ & $-\mathrm{F}$ & $-\mathrm{Cl}$ \\
\hline Triazolam & $-\mathrm{CH}_{3}$ & $=\mathrm{N}-$ & $-\mathrm{H}$ & $-\mathrm{Cl}$ & $-\mathrm{Cl}$ \\
\hline \multicolumn{1}{|c|}{ 1,4-Benzodiazepines } & $\mathbf{R}_{\mathbf{1}}$ & $\mathbf{R}_{2}$ & $\mathbf{R}_{\mathbf{3}}$ & $\mathbf{R}_{2}$, & $\mathbf{R}_{\mathbf{8}}$ \\
\hline 7-Amino-flunitrazepam (metabolite) & $-\mathrm{CH}_{3}$ & $=\mathrm{O}$ & $-\mathrm{H}$ & $-\mathrm{F}$ & $-\mathrm{NH}_{2}$ \\
\hline Chlordiazepoxide & ---- & $-\mathrm{NHCH}$ & $-\mathrm{H}$ & $-\mathrm{H}$ & $-\mathrm{Cl}$ \\
\hline Clonazepam & $-\mathrm{H}$ & $=\mathrm{O}$ & $-\mathrm{H}$ & $-\mathrm{Cl}$ & $-\mathrm{NO}_{2}$ \\
\hline Diazepam & $-\mathrm{CH}_{3}$ & $=\mathrm{O}$ & $-\mathrm{H}$ & $-\mathrm{H}$ & $-\mathrm{Cl}$ \\
\hline Flunitrazepam & $-\mathrm{CH}_{3}$ & $=\mathrm{O}$ & $-\mathrm{H}$ & $-\mathrm{F}$ & $-\mathrm{NO}_{2}$ \\
\hline Lorazepam & $-\mathrm{H}$ & $=\mathrm{O}$ & $-\mathrm{OH}$ & $-\mathrm{Cl}$ & $-\mathrm{Cl}$ \\
\hline Nordiazepam (metabolite) & $-\mathrm{H}$ & $=\mathrm{O}$ & $-\mathrm{H}$ & $-\mathrm{H}$ & $-\mathrm{Cl}$ \\
\hline Oxazepam (metabolite) & $-\mathrm{H}$ & $=\mathrm{O}$ & $-\mathrm{OH}$ & $-\mathrm{H}$ & $-\mathrm{Cl}$ \\
\hline
\end{tabular}

Table 1: $\mathrm{R}$ groups of benzodiazepines ${ }^{6}$ 


\subsubsection{Pharmacology}

Benzodiazepines have a well-recognized mechanism of action. These compounds act as central nervous system (CNS) depressants by binding to the receptors of one subtype of the neurotransmitter gama-amino butyric acid $(\mathrm{GABA})_{\mathrm{A}}$ and inhibiting the release of GABA. The $\mathrm{GABA}_{\mathrm{A}}$ receptor is a ligand-gated ion channel that is activated by GABA. The binding of benzodiazepines causes the inhibition of the release of GABA from the $\mathrm{GABA}_{\mathrm{A}}$ receptor resulting in the CNS depressant behavior of the drug by increasing the amount of ions transmitted through the channel (figure 3). ${ }^{87}$

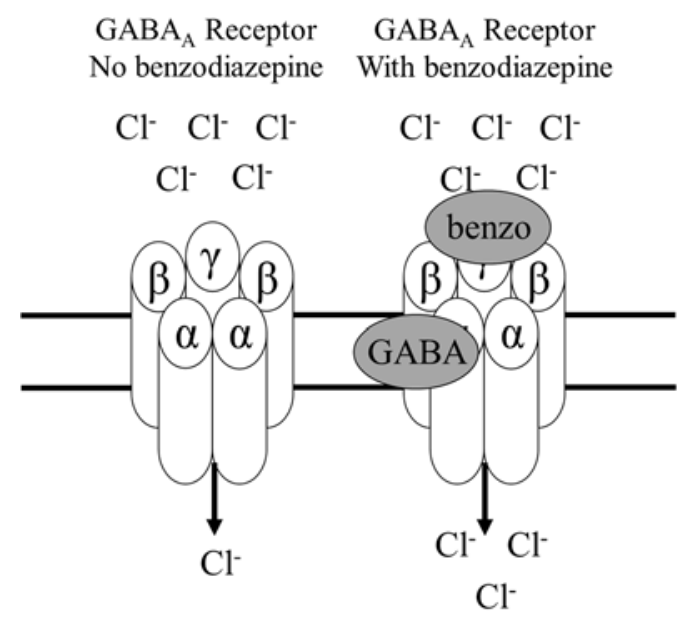

Figure 3: $\mathrm{GABA}_{\mathrm{A}}$ receptor without benzodiazepine and with benzodiazepine, note that GABA and the benzodiazepines bind at a different site causing GABA to continually bind and increasing the flow of ions through the channel ${ }^{5,87}$

When the benzodiazepine binds to the receptor it induces a conformational change in the receptor. This binding is separate to the binding pocket of the GABA agonist site. Benzodiazepines promote the binding of the inhibitory neurotransmitter GABA to the $\mathrm{GABA}_{\mathrm{A}}$ receptors. They do not directly activate the $\mathrm{GABA}_{\mathrm{A}}$ receptors but instead are positive allosteric modulators of the effects of GABA. ${ }^{87}$ The varied 
pharmacological uses of the drugs are due to the multiple subtypes of GABAA receptors that involve benzodiazepines. ${ }^{5}$ The structure of the benzodiazepine has an effect on the onset of action and duration of action. For the treatment of insomnia, hypnotic benzodiazepines are selected with a rapid onset but a longer duration of action than a benzodiazepine chosen to assist in anesthesia. ${ }^{5}$

There are three main methods for the administration of benzodiazepines, intravenously, intramuscularly, and the most common, orally. When taken orally, the drugs are completely absorbed by the body due to their high lipid solubility, but the rate of adsorption is dependent on the benzodiazepine. The first-pass effect, which occurs when the drug is metabolized in the gastrointestinal tract before entering the bloodstream, is generally significant with benzodiazepines prior to distribution. ${ }^{5}$ Benzodiazepines have a range of elimination half-lives, and the administered compound can be classified based on this property. Short-acting benzodiazepines, such as midazolam, have an elimination half-life less than 6 hours. Benzodiazepines with an elimination half-life between 6-24 hours are intermediate-acting. The group contains the majority of benzodiazepines including the most common, alprazolam. Finally, long-acting benzodiazepines have an elimination half-life exceeding 24 hours. This classification scheme does have complications due to the fact that many benzodiazepines produce active metabolites that may have a different elimination half-life than the parent drug. 5,7

The metabolism of benzodiazepines occurs mainly in the liver. Phase I metabolism of benzodiazepines follow four main routes: hydroxylation, deamination, reduction, and dealkylation, caused by the transformation of functional groups by the microsomal enzymes in the liver. ${ }^{5}$ Many of the major metabolites, such as nordiazepam 
and oxazepam are pharmacologically active and as previously mentioned, have an effect on the activity of the benzodiazepine administered. Metabolism pathways of a number of benzodiazepines are shown in Figures 4 and 5. Phase II metabolism of benzodiazepines mainly involves the conjugation with glucuronic acid, producing the major urinary products. $^{5}$

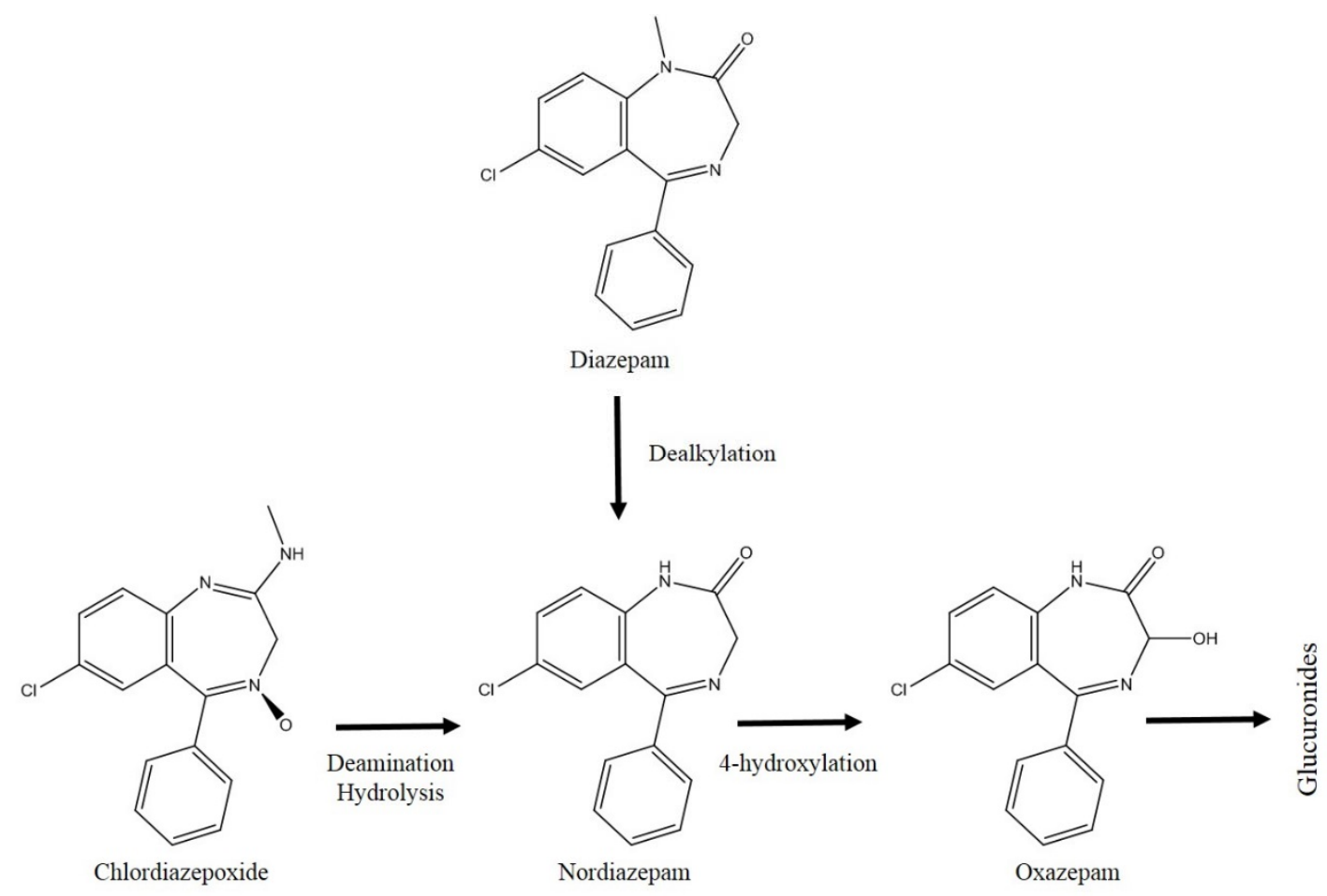

Figure 4: Routes of metabolism for some 1,4-benzodiazepines ${ }^{3} ; 1-4$ benzodiazepines can undergo dealkylation or deamination followed by hydroxylation and glucuronidation. 


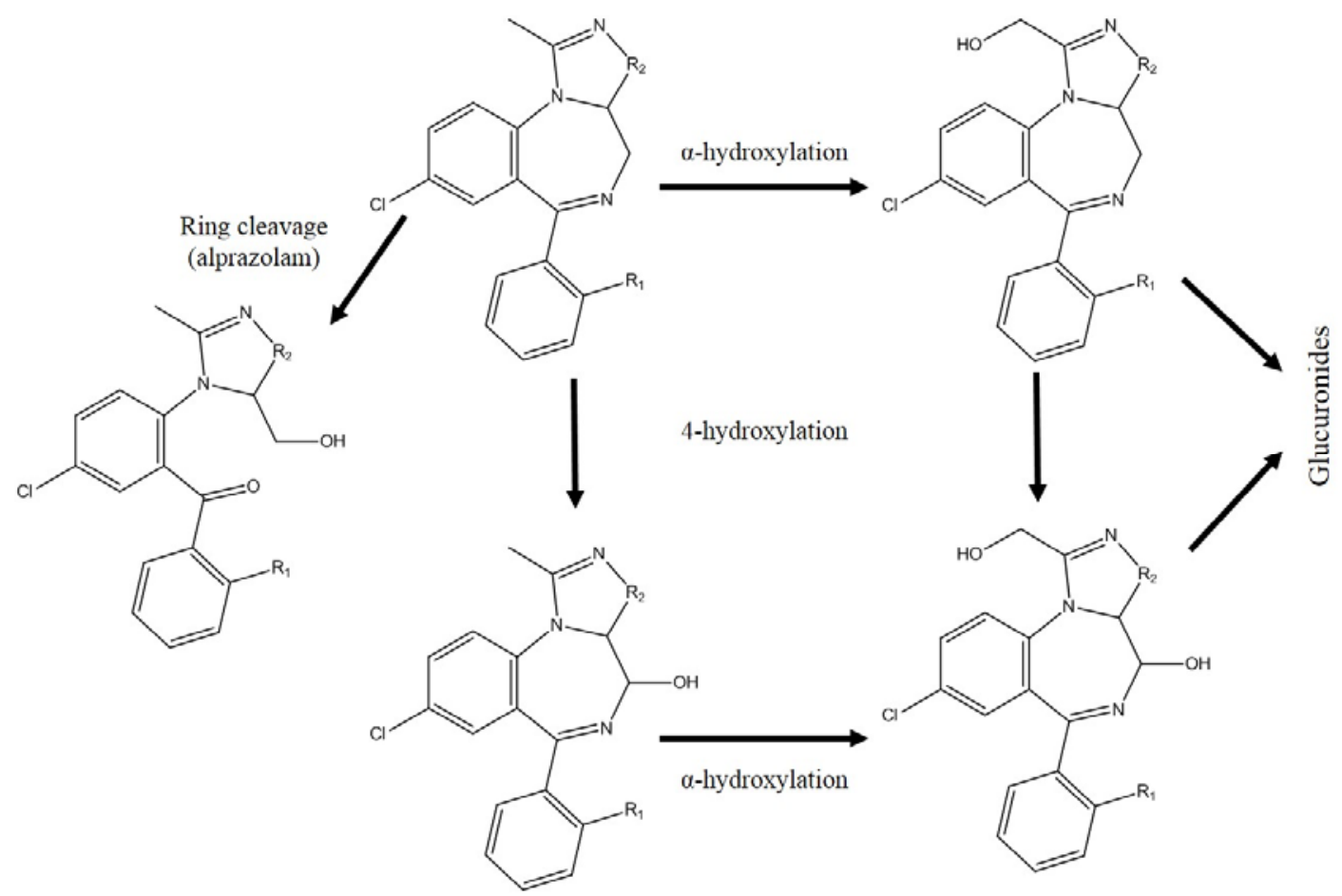

Figure 5: Routes of metabolism for 1,2-triazolo and 1,2-imidazo-benzodiazpines ${ }^{3}$; These groups of benzodiazepines can undergo ring cleavage or multiple hydroxylations followed by glucuronidation.

\subsubsection{Prevalence in Drug Facilitated Sexual Assaults}

Drug facilitated sexual assaults (DFSA) occur every day in the United States, with increasing frequency. A DFSA is defined as "the voluntary or involuntary ingestion of a drug by a victim that results in an act of sexual activity without consent." 8 The impairment effects differ from one victim to another, however, there is incapacitation as a result of consuming a substance common to these types of cases. Victims are often subjected not only to physical harm but also physiological harm, which is one of the main reasons these crimes are not reported immediately. ${ }^{8}$ The victim may not have any memory of the event, the person who committed the crime, or the circumstances surrounding the act. They also may have feelings of shame, guilt, and embarrassment 
and may not feel ready to report the crime to the authorities. ${ }^{8}$ The delay in reporting leads to reduced concentrations of drugs in body, thus, lowering the amount of drug present for an analyst to detect. This adds to the challenge of detecting drugs in DFSA, which are already administered at low concentrations and have fast clearance rates. ${ }^{8}$

Often victims are administered a drug unknowingly into food or beverages, in order for a criminal to incapacitate the victim. While Rohypnol ${ }^{\circledR}$ (flunitrazepam) and gamma-hydroxybutyrate (GHB) are frequently mentioned in the media, a wide variety of compounds are used in the commission of such crimes. ${ }^{9,10,11,12}$ Criminals in these cases chose a drug that creates the desired physiological effects on a victim such as confusion, drowsiness, impaired judgment and memory, and loss of consciousness, among others. ${ }^{10}$ Often the drugs chosen are central nervous system depressants, such as benzodiazepines, whose sedative effects can be enhanced in combination with ethanol. As reported by the International Narcotic Control Board, benzodiazepines rank amongst the most widely and frequently prescribed medications in the world ${ }^{4}$, making them readily available for use in DFSA. ${ }^{9-12}$ Therefore, the ability to detect these compounds is critical to toxicologists today in order to determine the type of drug used in the cases of DFSA. Recommendations have been made for the detection limits required for analyzing urine specimens using standard analytical methods such as gas chromatography-mass spectrometry or liquid chromatography-mass chromatography. The recommended maximum detection limit for one of the most common benzodiazepines, alprazolam is 10 $\mathrm{ng} / \mathrm{mL}$ and it was recommended that new techniques used for the determination of DFSA should meet this detection limit. ${ }^{11}$ 


\subsection{Current Methods of Detection}

Screening techniques are used by toxicologist in order to determine the type of drugs present in a biological sample prior to confirmation of the quantity and identity of the drugs. In terms of DFSA, the biological samples used are often urine, and many contain a vast variety of drugs, such as cocaine, nicotine, and other narcotics. ${ }^{8}$ This dissertation will discuss the screening techniques in terms of the detection of benzodiazepines and the complications that arise from their detection. Benzodiazepines when used in DFSA cause a number of analytical problems for the toxicologist. Depending on the circumstances, the concentration of these drugs that are present in urine may very low. This concentration is often less than $50 \mathrm{ng} / \mathrm{mL}$ due to a number of key factors. These compounds are prescribed in low dosages, therefore the amount administered will be dictated by the dosage of the source pill. Also many victims of these crimes do not report them immediately, allowing the drug to continue to be metabolized, reducing the amount present in urine. The screening process is further complicated due to the metabolism process as described previously, which gives rise to multiple metabolites. ${ }^{13,14,15,9,12,16,17,18,19}$

\subsubsection{Immunoassay}

Immunoassays are the screening tests most commonly utilized in toxicology laboratories. They provide rapid screening at a low cost and are relatively accurate. ${ }^{5}$ There are a number of types of tests which can be utilized such as enzymatic immunoassay $^{15}$, enzyme multiplied immunoassay technique ${ }^{15,9,18,20}$, kinetic interaction of microparticle in solution ${ }^{20,19}$, and radioimmunoassay. ${ }^{19}$ Each of these tests involve 
different enzymes and chemical reactions but all follow the same basic process. All tests employ specific antibodies produced in the presence of the target drug or drug class, diazepam for most benzodiazepine assays, which will bind to the drug when added to a biological sample. This binding will produce a measurable signal such as a change in absorbance (enzyme multiplied immunoassay technique, kinetic interaction of microparticle in solution) or change in radioactivity, radioimmunoassay. ${ }^{15,20,19}$

The enzyme multiplied immunoassay technique assay uses a drug-analog labeled enzyme in addition to the drug specific antibody which are mixed and added to the sample on a substrate of nicotinamide adeten dinucleotide (NAD). When drug is present, it binds to the antibody preventing the drug-analog labeled enzyme from binding, making an active enzyme which converts NAD to its product increasing absorbance. If no drug is present the drug-analog labeled enzyme binds to the antibody, effectively preventing enzyme activity and producing no change in absorbance. The change in absorbance shows a positive detection of the drug of interest. Figure 6 shows the reaction of the assay with and without the presence of the target analyte. ${ }^{20}$ 


\section{No Drug:}

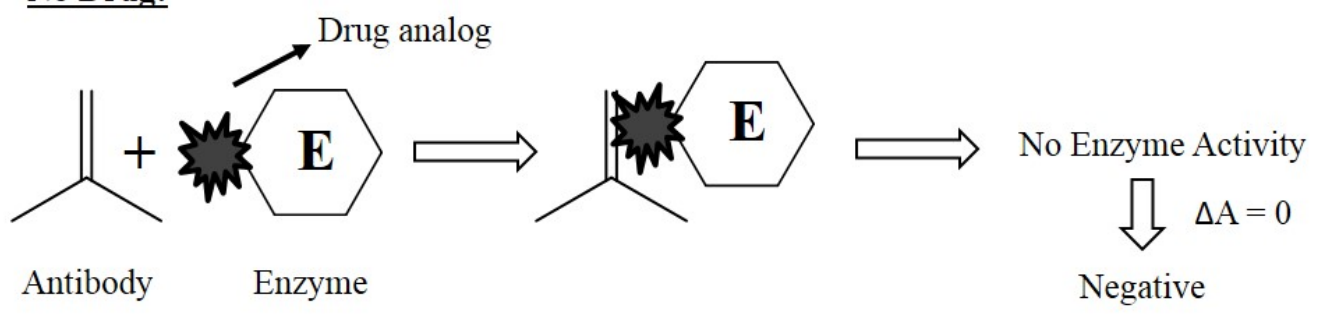

\section{Addition of Drug:}

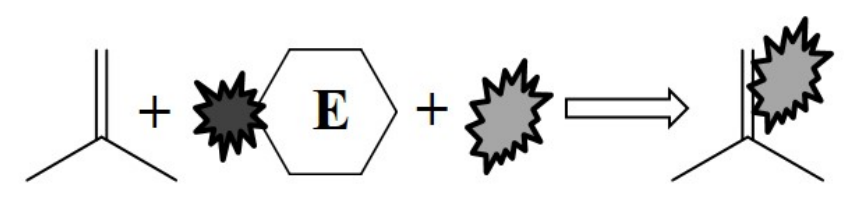

Antibody

Enzyme

Drug

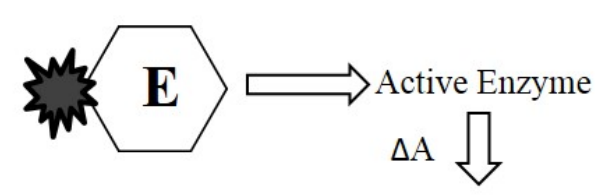

Positive

Figure 6: Theory of enzyme multiplied immunoassay technique assay; ${ }^{2}$ When there is no drug present the antibody will bind with the drug analog which is attached to an enzyme. This process blocks the ability of the enzyme to catalyze a subsequent colorimetric reaction. When drug is present, the antibody binds the target drug, freeing the enzyme conjugate, allowing enzyme activity to occur. ${ }^{20}$

When screening for benzodiazepines in urine, additional sample preparation is often required due to the high presence of glucuronidated conjugates over free drug in urine. $^{5,3,10}$ These conjugates reduce sensitive in immunoassays as a result of the presence of both the undetectable conjugate and the free drug. In order to remove the drug from the conjugate, enzyme hydrolysis with glucuronidase, often a one to two hour process, is recommended..$^{10,9,12,17}$ Hydrolysis steps are often integrated into the kits that are sold for toxicological screening. ${ }^{18}$ In general, enzymatic hydrolysis is also recommended due to problems with an alternative procedure, acid hydrolysis, which can promote the conversion of certain benzodiazepines and metabolites into their 
corresponding benzophenone. These benzophenones are formed due to the cleavage of the diazepine ring at the $\mathrm{N}-4$ position, causing an open ring structure which can reduce the specificity of the immunoassay. ${ }^{10,21}$

Once the sample is prepared, the immunoassay process is performed using an automated plate reader. Careful attention to the quality and quantity of the sample is important as false negatives or false positives can occur, which result from matrix interferences and the presence of other drugs in the patients' system. Cross-reactivity is a problem that results from the tendency of an assay to respond to compounds other than the one the assay is designed to detect. An ideal immunoassay should have high crossreactivity with structurally similar compounds when designed to detect a class of drugs to prevent false-negatives and a low cross reactivity with other structurally similar compounds not part of the class of drugs to prevent false-positives. ${ }^{5}$ As previously mentioned, benzodiazepine assays commonly use antibodies that are specific for diazepam. Because of structural differences with diazepam, certain benzodiazepines are poorly detected by these antibodies, resulting in false negative assays. This was demonstrated by Augsburger et al. who found that urine samples which tested positive for lorazepam using gas chromatography/ mass spectrometry (GC/MS) could not be detected using standard immunoassay methods, developed with diazepam haptens. ${ }^{19}$ A later comparison of four different immunoassays for the detection of lorazepam found that none gave a positive result prior to hydrolysis, and only three gave some positive results after hydrolysis. ${ }^{22}$

Low cross reactivity is not the only limitation of immunoassay screening. Due to the variations in cross reactivity, many samples can also produce false negatives during 
screening. This is particularly true for drugs that are administered in the commission of DFSA as the victim may not report the crime for several days following the attack, and low doses of more potent benzodiazepines may have been used, further increasing the difficulty of detection. Many immunoassays have cut-off levels between 50-300 ng/mL. ${ }^{5,10,12,19-20}$ These cut offs are utilized to detect benzodiazepines at therapeutic concentrations $(0.05-2 \mathrm{mg} / \mathrm{mL})^{5}$ and minimize false positives. Unfortunately the concentrations that may be observed in cases of DFSA can be lower. ${ }^{8}$ As mentioned above it has been suggested that diazepam detection should have a maximum detection limit of $10 \mathrm{ng} / \mathrm{mL}^{11}$ To overcome these limitations of immunoassays, many toxicology laboratories have turned to GC/MS for screening and confirmation. However, many hospitals and smaller clinical laboratories may not have access or training in this technology for sample screening.

\subsubsection{Gas chromatography mass spectrometry}

Gas chromatography interfaced with mass spectrometry (GC/MS) is utilized by toxicology laboratories to allow for screening of a variety of drugs all at the same time. It is often referred to as the "gold standard" for urine toxicology testing due to its specificity and selectivity. ${ }^{15}$ There are still some limitations to GC-MS as a screening method for benzodiazepines including the problems of added sample processing through derivatization and longer analysis times when compared to immunoassay.

Gas chromatography uses a carrier gas mobile phase to move volatile analytes through a chemically treated thermostated column (stationary phase). Separation of the analytes occurs through partitioning between the mobile and stationary phases and are detected, in this case, by the mass spectrometer. The drugs of interest must be volatile in 
order for separation to occur. In the case of benzodiazepines, they are not volatile and must be derivatized after extraction in order to be analyzed by GC/MS. There are a number of different methods for derivatization. Propylation followed by propinylation ${ }^{23}$, acetylation ${ }^{19}$, and silyation ${ }^{9}$ are some common derivatization methods to produce volatile products. The products of these reactions must be well known in order to observe them through mass spectrometry.

Mass spectrometry uses an ionization source to apply a charge to the volatile analytes as they move into the mass spectrometer. Dependent on the ionization source, the ion will break apart into fragment ions which are separated in the mass analyzer by their mass to charge ratio and detected. ${ }^{26}$ The fragmentation patterns observed are consistent with a particular analyte and the drugs of interest can be identified. The combination of GC/MS allows not only for the screening and identification of unknown drugs in a toxicology sample but is also sensitive enough to quantify. ${ }^{5}$

A number of studies have used GC/MS to confirm the presence of benzodiazepines in urine samples which have been screened by immunoassays. ${ }^{9}, 15,19,23$ Following acid hydrolysis and acetylation, GC/MS was performed to detect the various benzophenone and acetylated benzophenone products of a variety of consumed benzodiazepines. Detection limits observed ranged from $2.5-250 \mathrm{ng} / \mathrm{mL}$ depending on the derivative detected and compounds not detected using immunoassays (lorazepam) were detected using GC/MS. ${ }^{19}$ After extraction from blood samples and a two-step derivatization of propylation followed by propionylation, GC/MS analysis was found to have limits of detection between $0.5-60 \mathrm{ng} / \mathrm{mL}$ and separation of 23 benzodiazepines and derivatives, however total time of analysis including sample preparation was two hours. ${ }^{23}$ 
While GC/MS is considered the "gold standard" it does have limitations including the time of analysis and the detection of derivatives rather than the parent benzodiazepine.

\subsection{Surface Enhanced Raman Spectroscopy}

The present dissertation proposes a rapid screening method for the screening of benzodiazepines in urine using surface enhanced Raman spectroscopy (SERS). As previously discussed, current methods of screening have a number of issues. Immunoassays have high cut-off levels and poor cross-reactivity with certain benzodiazepines. They only provide information on whether or not a benzodiazepine is present, but cannot determine which particular benzodiazepine has been detected. Sample preparation can also be time consuming due to the need for hydrolysis steps. Immunoassays do have the benefit of automatic readers and they are not very expensive. GC/MS on the other hand is an expensive procedure, both in terms of equipment and us of the personal, especially when used in a hospital setting, where access to advanced analytical techniques and trained staff is limited. Gas chromatography mass spectrometry also requires an additional sample preparation step involving derivatization of the benzodiazepine in order to improve stability and volatility. Many of these disadvantages can be overcome with SERS. This method provides limits of detection below the 10 $\mathrm{ng} / \mathrm{mL}$ upper limit in DFSA samples and a rapid analysis time from sample preparation to detection of less than 30 minutes. In addition, SERS also provides information about the identity of an individual benzodiazepine instead of the generalized detection of a class of compounds through immunoassays. Also, this technique is relatively inexpensive when compared to GC/MS. Before discussing the development of this technique, the basic principles of Raman spectroscopy as well as SERS must be understood. 


\subsubsection{Raman spectroscopy}

Raman scattering or effect was first described by C.V. Raman in 1928 as a "new type of secondary radiation". ${ }^{24}$ He was able to observe what are now known as the antiStokes Raman scattering by eye in a number of organic liquids, noting that the increase in energy emitted was different than other optical processes, such as fluorescence, which were already well known. ${ }^{24}$ Raman spectroscopy measures the scattering of photons that arise from the Raman effect.

Raman spectroscopy is a type of optical spectroscopy that involves the absorption of one photon (incident) and emission of a second photon (scattered) simultaneously, this process is known as scattering. It is achieved by irradiating a sample molecule with a powerful laser source of visible or near-infrared (IR) monochromatic radiation. There are two main groups of scattering processes, elastic and inelastic. Elastic scattering is where the incident and scattered photons have the same energy (E), leaving the molecule at the same energy level after the scattering has occurred (Rayleigh scattering), and does not reveal any structural information about a molecule. Inelastic scattering is where the incident and scattered photons are at different energy levels. ${ }^{25}$ The transition between the two states in the molecule relates to the energy difference. Raman scattering is inelastic scattering which involves transitions between the vibrational/rotational energy levels.

When no electronic transitions exist in a molecule, Raman scattering can still occur because it does not require direct absorption of a photon to a real higher energy state. In these cases, the molecule is excited to a virtual state. Compared to other forms of optical spectroscopy, such as absorption and fluorescence, scattering is a weak phenomenon. ${ }^{25}$ Raman scattering can be subdivided by the energy of the incident photon in relation to 
the scattered photon. In the Stokes process, the scattered photon has less energy than the incident photon, while in the anti-Stokes process, the scattered photon has more energy than the incident photon. ${ }^{25}$ These processes can be described in a simplified Jablonski diagram in Figure 7.

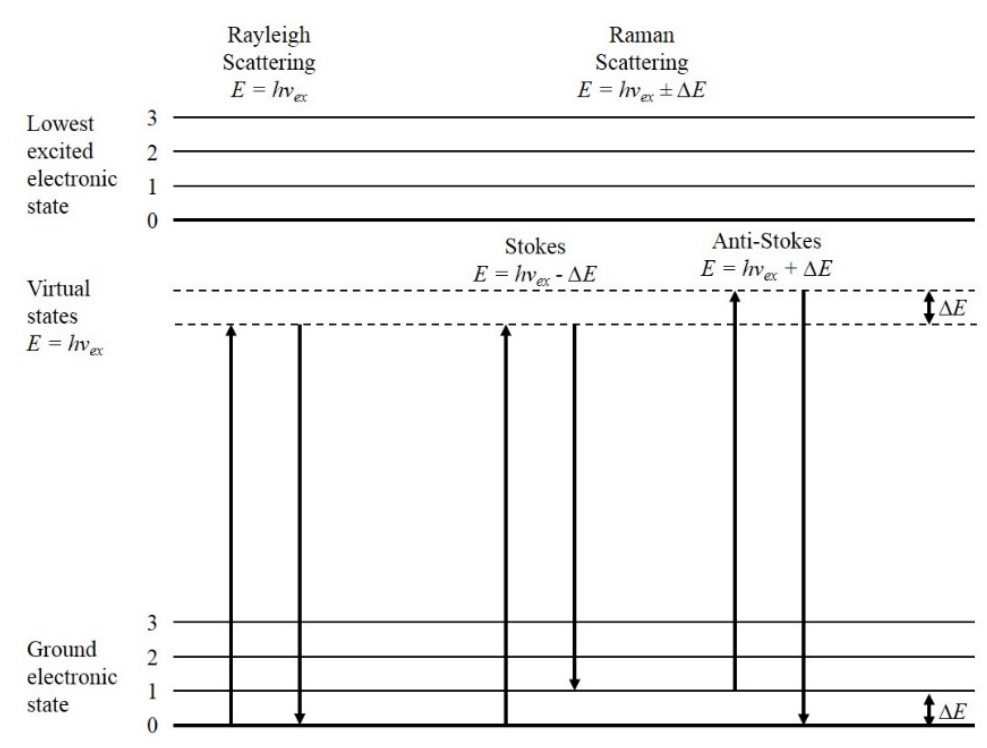

Figure 7: Simplified Jablonski diagrams for Rayleigh, Stokes, and anti-Stokes processes $^{25-26}$. In Rayleigh scattering the energy difference is zero, while for the Stokes process the emitted energy is less than the incident and for the anti-Stokes process the emitted energy is greater than the incident.

Raman scattering does not just rely on the energy difference between the incident photon and the emitted photon, but also on the polarizability or the ability to induce a dipole in a molecule. ${ }^{25-26}$ To understand the polarizability, imagine a molecule in a static electric field. This molecule is structurally characterized by the positions of the atoms and electrons present and their interactions with one another are in equilibrium. When an external electric field is applied, like a laser, the constituents positions and properties can be modified resulting in a change of the dipole moment of the molecule, causing an 
induced dipole moment. These changes depend on the direction and magnitude of the electric field applied. Polarizabilty is a measure of the responsiveness of the electrons in a molecule in the presence of an external electric field. Generally organic molecules, such as drugs, are highly polarizable due to the large number of free electrons. Taking into account polarizability, the Raman scattering can be described as the production of Raman scattered light by an induced Raman dipole. ${ }^{25}$

Raman instrumentation measures the energies of the scattered photons in relation to the energy of the incident photons from a monochromatic laser source in the visible or near-IR range. The Raman spectra produced consists of all three types of scattering previously described. Of these, the strongest type of scattering is Rayleigh scattering. The Stokes and anti-Stokes scattering are much weaker are with the intensity of the Stokes scattering being greater than that of the anti-Stokes scattering. These two forms of scattering are opposite but identical. However, since the Stokes scattering is more intense, it is generally is the only part of the spectrum used..$^{25-26}$

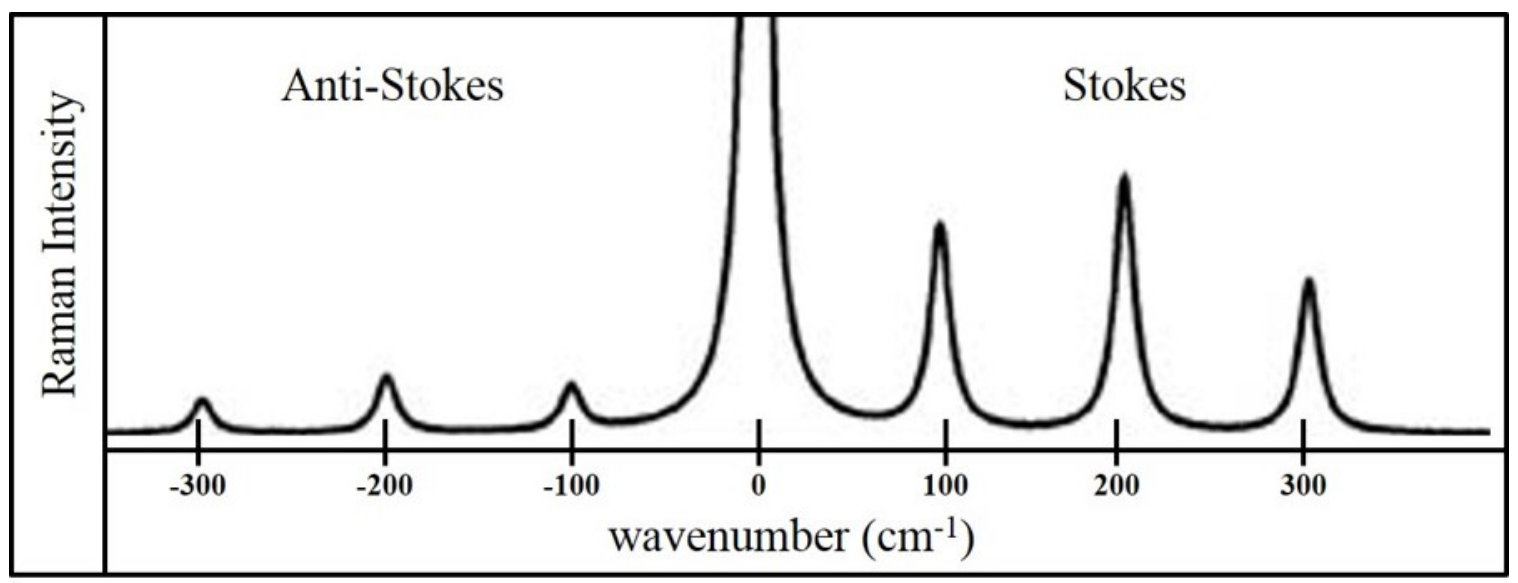

Figure 8: Example Raman spectra ${ }^{25-26}$. The Stokes and anti-stokes scattering occur at the same wavenumber but the Stokes shift is of higher intensity 
The Raman spectrum corresponds to the relative Raman intensity as a function of the Raman shift measured in wavelength or energy (generally wavenumber $\mathrm{cm}^{-1}$ ). The source irradiates the sample and scattering is measured by a charge coupled device (CCD) detector at $90^{\circ}$ to the incident radiation to prevent viewing of the source radiation. The laser wavelength selected, in this study a $785 \mathrm{~nm}$ diode laser, is important to prevent fluorescence problems in the sample as well as absorption that may occur in colored samples. ${ }^{26}$ However, it is important to note that the wavelength of excitation does not affect the magnitude of Raman shifts. The same compound will display identical Raman shifts regardless of the laser wavelength. ${ }^{25-26}$

The Raman spectrum of a molecule contains peaks that correspond to the vibrational modes of a molecule, similar to infrared spectroscopy. In fact, the infrared vibrational spectrum and Raman spectrum of a molecule should have a number of identical spectral peaks that correspond to vibrational modes in the molecule that are both IR and Raman active. Differences do occur however when a particular mode in the molecule is only Raman active. The Raman shift of a peak is equal to vibrational energy of the mode and the total intensity scattered by a given vibrational mode. The frequency of vibrations can be determined through a quantum treatment, assuming that the transitions in vibrational energy levels are brought about by the absorption of radiation, and that the provided energy exactly matches the difference in the energy levels $(\Delta \mathrm{E})$ between the vibrational quantum states. This occurs when the vibration causes a change in the dipole moment. ${ }^{26}$ Using the quantum treatment, the wavenumber of a given bond in a molecule can be calculated based on the expected vibrational frequency for a particular bond in a molecule, see Equation 1. 


$$
v=\frac{1}{2 \pi c} \sqrt{\frac{k}{\mu}}
$$

Equation 1: where $v$ is the wavenumber of an absorption maximum $\left(\mathrm{cm}^{-1}\right), k$ is the force constant for the bond $(\mathrm{N} / \mathrm{m}), c$ is the velocity of light $\left(\mathrm{cm} \mathrm{s}^{-1}\right)$, and $\mu$ is the reduced mass $(\mathrm{kg})^{26}$

As a whole the Raman spectrum of a molecule will produce a unique molecular fingerprint related to the vibrational modes of the molecule. ${ }^{25-26}$ How these vibrational modes are determined and spectral peaks are identified will be further discussed in Chapter 7.

One of the benefits of using Raman over IR spectroscopy is that water does not interfere with the Raman spectra of compounds, allowing Raman analysis to be performed in aqueous samples, such as urine. The Raman spectra of water is weak and featureless, resulting from short-lived, picosecond, collision-induced spontaneous Raman scattering processes. ${ }^{27}$ Because vibrational scattering produced by other molecules lies well above this baseline, minimal interference occurs. ${ }^{27}$

As previously stated, Raman spectroscopy is not a sensitive technique, suffering from poor signal to noise ratios and low concentration sensitivity. To overcome this low sensitivity problem and allow for analysis of low concentration samples, especially those in solution, surface enhanced Raman spectroscopy, which can increase the intensity of a spectra several orders of magnitude, has been employed.

\subsubsection{Mechanism of SERS}

Surface enhanced Raman spectroscopy (SERS) was discovered by Fleischmann et al. (1974), who observed an intense Raman scattering from pyridine absorbed onto a 
roughened silver electrode. It was first thought that this was due to a large number of pyridine molecules being absorbed on the electrode surface. ${ }^{28}$ Two independent research groups demonstrated that the increased intensity Fleischman observed was the result of an enhancement of the scattered intensity while the molecules were absorbed onto the silver surface and not the effect of local increases in concentration. ${ }^{29,30}$ However, the two groups could not agree upon what caused the SERS mechanism and two main theories were developed and eventually combined. In the simplest terms, SERS is the amplification of the Raman signals of molecules by several orders of magnitude through the interaction of the electromagnetic radiation with a metal surface that is in close proximity or adsorbed to the molecules. ${ }^{25}$ Physically, the main difference between SERS and traditional Raman spectroscopy is the addition of a metal surface or substrate. There are two components that contribute to the activity of SERS, electromagnetic and chemical enhancement. However, the main contributor to the SERS mechanism is due to the electromagnetic enhancement.

In order to discuss the SERS process in more detail, first, the optical properties of coinage metals, specifically gold $(\mathrm{Au})$ and silver $(\mathrm{Ag})$, which make up the SERS substrate must be discussed. Optical properties of bulk metals are characterized by their dielectric function $\epsilon(\omega)$. The dielectric function is related to the index of refraction, $n(\omega)$, by the equation $n(\omega)=\sqrt{\epsilon(\omega)} \cdot{ }^{31}$ Each function depends on the frequency $(\omega)$ of the light because most materials respond differently when different frequencies (wavelengths, $\lambda$ ) of electromagnetic radiation are applied to the material. Therefore, the dielectric function can either be represented as a function of $\omega$ or $\lambda .^{31,25}$ When examining the optics of material objects such as prisms and lenses, both the dielectric 
function and the index of refraction are positive real numbers, however, in reality, the dielectric function of materials which are not transparent at a given wavelength will be a complex number. The metals used in SERS have a dielectric function that is complex. ${ }^{32}$

The dielectric functions of $\mathrm{Au}$ and $\mathrm{Ag}$ are shown in Figure 9. Both the real (a) and imaginary (b) parts of the dielectric function, $\epsilon(\lambda)$, for each metal are represented from $300-900 \mathrm{~nm}$ to show the characteristics between the near-UV and near-IR wavelengths. ${ }^{31}$ There are a number of generalized characteristics of the real and imaginary parts of the bulk dielectric function for both metals.

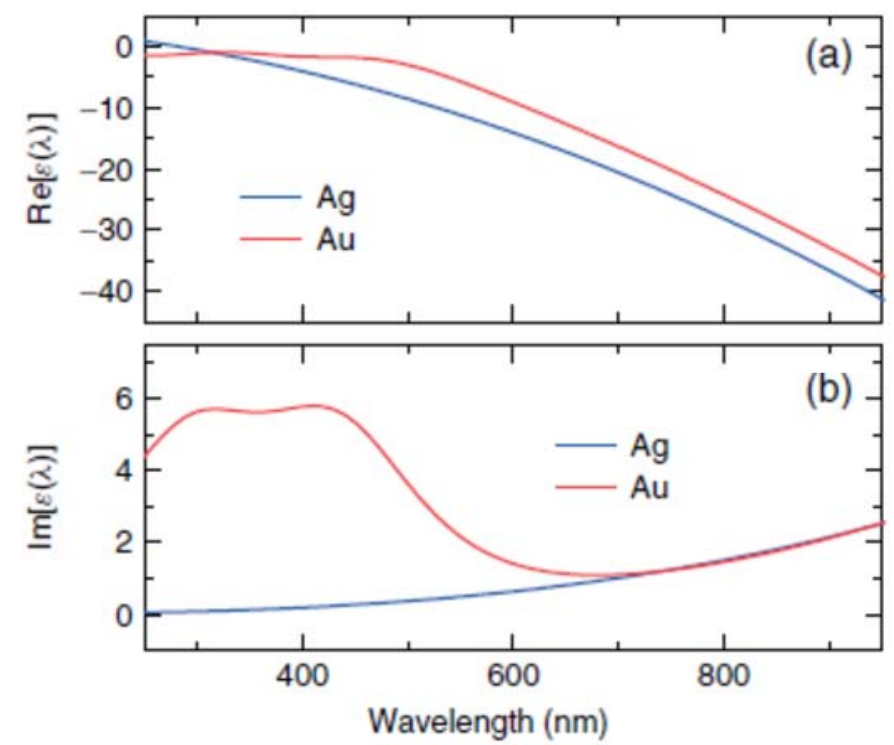

Figure 9: The real (a) and imaginary (b) parts of the dielectric function for Ag and Au. The imaginary part (b) is positive and of smaller magnitude while the real part (a) is negative in the visible range. ${ }^{31}$

For most of the visible range, the real part of the dielectric function of both metals is both large and negative. This is one of the main reasons that $\mathrm{Ag}$ and $\mathrm{Au}$ are useful as plasmonic materials. If the imaginary parts of $\epsilon(\lambda)$ are ignored, it can be said that the 
real parts at long wavelengths follow a simple model for the dielectric function of a metal, the lossless Drude model, which predicts an approximately $-\lambda^{2}$ dependence for the real part at long wavelengths. ${ }^{25,31}$ The lossless Drude model predicts a dielectric function in the following form:

$$
\epsilon=\epsilon_{\infty}\left(1-\frac{\omega_{p}^{2}}{\omega^{2}}\right)=\epsilon_{\infty}\left(1-\frac{\lambda^{2}}{\lambda_{p}^{2}}\right)
$$

where $\omega_{p}=2 \pi c / \lambda_{p}$ is the plasma frequency (for Au and Ag $\sim 280 \mathrm{~nm}$ ) of the metal

The similarity in the real portion of the dielectric function (Figure 8a) of both $\mathrm{Ag}$ and $\mathrm{Au}$ shows that these two metals have very similar electronic densities. This is the approximate downturn of the real part of the dielectric function observed at longer wavelengths.

The imaginary part of the dielectric function $\epsilon(\lambda)$ cannot be ignored. In reality, bulk metals are not lossless. While $\operatorname{Im}[\epsilon(\lambda)]$ is smaller than the real counterparts for both metals for most of the visible range, the effects of the imaginary portion of the function are important. The imaginary portion is related to the absorption of the material (if the function is equal to zero, then the material does not absorb light). ${ }^{31}$ For sliver, the imaginary portion of the dielectric function can be generalized using the lossless Drude model. For gold, this is more complicated because $\epsilon(\lambda)$ has additional contributions from other electronic transitions in the electronic band structure of gold. ${ }^{31}$ These interband electronic transitions cause the observed "double hump" structure in the imaginary portion of $\epsilon(\lambda)$ for $\mathrm{Au}(\sim 400 \mathrm{~nm})$ and the relatively higher absorption of $\mathrm{Au}$ (with respect to $\mathrm{Ag}$ ) for wavelengths less than $600 \mathrm{~nm}$ (Figure 8b). For wavelengths 
greater than $600 \mathrm{~nm}, \mathrm{Au}$ and $\mathrm{Ag}$ are comparable for the imaginary parts of $\epsilon(\lambda)$, therefore, both materials are similar in their electromagnetic responses in the near- and far-IR range. ${ }^{31}$

Once the dielectric function $\epsilon(\lambda)$ is known, the electromagnetic properties of the metal can be calculated at different geometries. This discussion will start with the simplest geometry, planar. The normal reflectance (R) of $\mathrm{Au}$ and $\mathrm{Ag}$ are plotted in Figure 10 using the complex dielectric functions previously shown. Gold has a reflectance of approximately $50 \%$ for wavelengths less than $600 \mathrm{~nm}$, while silver is highly reflective, approximately $100 \%$, over the entire visible range. This is the reason for the color of planar gold, yellowish/red. ${ }^{31}$

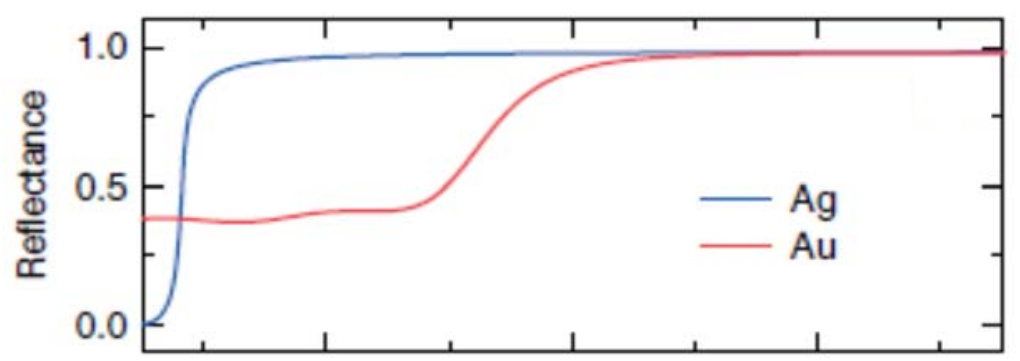

Figure 10: Reflectance at normal incidence for Ag and Au using the dielectric function shown in Figure $9^{31}$

The local field intensity enhancement factor (LFIEF) at the surface must also be examined to discuss the SERS mechanism. The LFIEF is described as how much the intensity of the electromagnetic field is changed with respect to the intensity that would have been observed at the same point without the metal. The local field intensity at a given point is proportional to the square of the electric field amplitude at that point: $|E(r)|^{2}$. The normalized value of $|E(r)|^{2}$ with respect to the intensity of the incoming 
field at a specific point $\left(\left|E_{0}(r)\right|^{2}\right)$ is the local field intensity enhancement factor at that point. $^{31}$

$$
\operatorname{LFIEF}(r)=|E(r)|^{2} /\left|E_{0}(r)\right|^{2}
$$

The local field intensity enhancement factor expresses the change in local intensity at a specific point produced by the presence of objects which interrupt the electric field of light. Therefore, any optical technique which depends on the intensity of light at a specific point (SERS) will be linked to the local field intensity enhancement factor. It can be noted that the enhancement or quenching of an optical process depends on whether the local field intensity enhancement factor is greater than or equal to one, respectively. ${ }^{31}$ The local field intensity enhancement factor also depends on $\omega$ (or $\lambda$ ), due to the local field at a specific point depend on $\omega$ and can be rewritten as:

$$
\operatorname{LFIEF}(r, \omega)=|E(r, \omega)|^{2} /\left|E_{0}(r, \omega)\right|^{2}
$$

Another aspect of basic electromagnetic theory is the local field intensity enhancement factor at a flat surface for normal incidence and is solely determined by the dielectric function. The local field intensity enhancement factor on the surface at the interface between the metal an air for both gold and silver are plotted in Figure 10. An ideal lossless metal with $100 \%$ reflectivity will create a field on the surface, which will cancel out the incoming field and the transmitted field. This sends the impacting electromagnetic wave back in the opposite direction from where it came. Therefore, a low local field intensity enhancement factor at the surface is a consequence of high reflectivity. In reality, the cancelation is not complete but is enough to produce a low local field intensity enhancement factor on the surface. Figure 11 shows that the local field intensity enhancement factor is less than one across the visible range for gold, thus 
showing a quenching in the intensity with the presence of gold. Silver produces a local field intensity enhancement factor greater than one when the wavelength is less than 400 $\mathrm{nm}$, where Ag stops being a good deflector. ${ }^{31}$

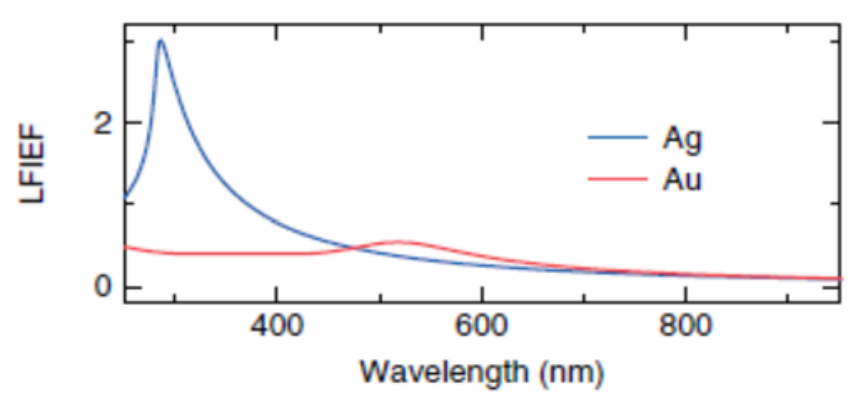

Figure 11: Local field intensity enhancement factor (LFIEF) at the surface of the metal for $\mathrm{Ag}$ and $\mathrm{Au}$ (at normal incidence). Generally the LFIEF is less than 1 across the visible range. ${ }^{31}$

Aside from the highly reflective properties of these metals, it may seem that there is no major advantage to using these metals as photonic materials. Molecules chemisorbed on a flat interface on these metals do not show much surface enhancement of the electromagnetic field when compared to other materials. The usefulness of these metals is related to the shape and orientation of the metal surface and its effects on local electric fields.

To discuss these effects and their impact on the three dimensional spherical particles used in the present study, discussion must be made of the electromagnetic field around a cylinder with polarization $\mathrm{E}_{\mathrm{i}}$. Maxwell's equations must be solved subject to the boundary conditions which have previously been done for a number of simple 
geometries to determine what happens to the field. ${ }^{25,33}$ For these purposes an approximation scheme, the electrostatic approximation, will be examined.

The electrostatic approximation can be represented schematically for a cylinder (Figure 12a). The problem is solved at different $\omega$ 's ( $\lambda$ 's) using the complex dielectric function of the material at that wavelength. The electrostatic approximation ignores the presence of the wavevector $k$ (or the wavelength $\lambda=2 \pi / k$ ) seen in Figure 12. The applied electric field is uniform and oscillating up and down with frequency $\omega$, instead of having a "wavelength". This approximation works when the size of the object is much smaller than the wavelength. Typically, the approximation will mostly be valid for objects in the size range of $10 \mathrm{~nm}$ or smaller when the wavelengths used are between 500-600 nm. ${ }^{31}$ It has also been used in some cases with even large objects that are beyond the size of these limitation. ${ }^{34}$

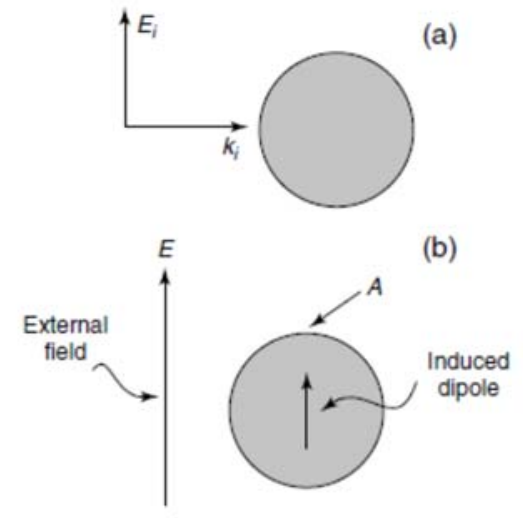

Figure 12: Representation of localized surface plasmon resonances of a cylinder (a) a cylindrical metal object is impacted by an electromagnetic wave coming from the side with wavevector $\mathrm{ki}_{\mathrm{i}}$ and polarization $\mathrm{E}_{\mathrm{i}}(\mathrm{b}) \mathrm{A}$ constant electric field in the direction of polarization with no wavevector. Point A is the boundary where the external field and the induced dipole are superimposed. ${ }^{31}$ 
The exact solution of a cylinder with dielectric function $\epsilon(\lambda)$ can be determined by a number of factors. The boundary conditions on the surface of the cylinder can be satisfied exactly by superimposing the external field $E$ with an induced dipole centered at the origin (Figure 12b). For points outside the cylinder, the electrostatic field looks like the external field superimposed with the dipole $(p)$ at the origin. ${ }^{31}$ The magnitude of the induced dipole that solves the electrostatic problem is proportional to:

$$
p \propto\left(\frac{\epsilon(\lambda)-\epsilon_{M}}{\epsilon(\lambda)+\epsilon_{M}}\right)
$$

The most important detail of the proportionality in Equation 5 is the denominator. As discussed previously $\epsilon(\lambda)$ is a complex number, thus, the full condition $\epsilon(\lambda)=-\epsilon_{M}$ cannot be satisfied exactly $(p \rightarrow \infty)$. At the wavelength where the real part of $[\epsilon(\lambda)]=$ $-\epsilon_{M}$, which is the case for metals such as Ag, the magnitude of $p$ will only be limited by how small the imaginary part of $\epsilon(\lambda)$ is. This is observed as a resonance called the dipolar localized surface plasmon. This resonance is induced solely by the shape of the object and the satisfying of boundary conditions. Objects with different shapes will have different resonances. Because metals have negative $\operatorname{Re}[\epsilon(\lambda)]$ spanning a wide range of magnitudes (Equation 1and Figure 9), this makes them ideal for satisfying a wide range of resonance conditions that occur in the solutions of the electromagnetic responses of many objects. ${ }^{31}$

When the geometry is changed to a metallic sphere, exact solutions of Maxwell's equation (Mie theory) or approximate solutions can be used. As with the cylinder, the electrostatic boundary conditions on the sphere are satisfied exactly by superimposing the 
induced dipole at the origin $p$ with the applied external field $E .^{31}$ The difference is due to the 3D nature of the sphere that the magnitude of the induced dipole is proportional to:

$$
p \propto\left(\frac{\epsilon(\lambda)-\epsilon_{M}}{\epsilon(\lambda)+2 \epsilon_{M}}\right)
$$

The resonance condition in the denominator has now changed, $\epsilon(\lambda)=-2 \epsilon_{M}$, and cannot be satisfied exactly due the presence of the imaginary portion of the dielectric function which is limited by how small $\operatorname{Im}[\epsilon(\lambda)]$ is at that wavelength.

If the surface of the sphere was to be examined, the places with the largest local fields are the two points along the axis that goes through the center of the sphere. This axis is oriented in the direction of the external field. Figure 13 show the axis in the sphere and the point labeled A is one of the largest local field points. These are the two points where the induced dipole and the external field add up due to the superposition and approach a maximum. When the local field intensity enhancement factor is calculated for point $\mathrm{A}$ on the sphere using the dielectric functions as a function of wavelength for both silver and gold, the resulting graph in Figure 13 is produced. Note that the scale of the local field intensity enhancement factor is much larger than that for planar surfaces of these metals seen in Figure 11, due to the presence of the metal sphere. ${ }^{31}$ The local field intensity enhancement factor chart shows the intensity enhancement that a molecule would experience if it was located at that position on the sphere. The peaks that are observed for both $\mathrm{Ag}$ and $\mathrm{Au}$ appear at the wavelength that the condition $\operatorname{Re}[\epsilon(\lambda)]=$ $-2 \epsilon_{M}$ is met. Silver has a stronger and narrower local field intensity enhancement factor when compared to Au because the condition is satisfied in $\mathrm{Ag}$ at a wavelength where the imaginary part of the dielectric function is much smaller than that of Au at its resonance 
frequency. This is why Ag is generally a better metal to use when compared to Au due to gold's broad, weaker resonance. This shows how intensities can be increased by large factors and can be tuned by changing the geometrical aspects of the metal. ${ }^{31}$

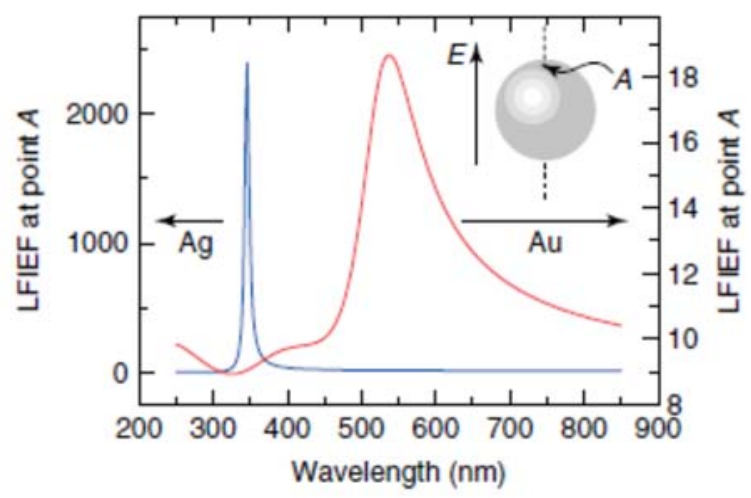

Figure 13: Local field intensity enhancement factor at point $\mathrm{A}$ on a sphere of $\mathrm{Au}$ or $\mathrm{Ag}$ in the electrostatic approximation. The largest local field intensity enhancement factors occur at the main symmetry axis in the direction defined by the polarization of the electric field. ${ }^{31}$

When using the electrostatic approximation the size of the sphere does not matter, however, when the size is between $30-100 \mathrm{~nm}$ the size is an appreciable percentage of the Raman excitation wavelength. Therefore, there are a number of effects related to the size of the object. First, dipolar localized surface plasmon resonances red shift as the size of the object increases. ${ }^{31}$ Dipolar localized surface plasmon resonances are also damped as size increases resulting in the broadening of the resonance and decrease in the local field intensity enhancement factor. Lastly, larger sized objects can produce size-induced resonances that do not exist in the smaller size range. These are related to the activation of multipolar resonances that do not couple to light effectively. ${ }^{31}$ To avoid these issues it is best to keep the size of the object as small as possible, below $30 \mathrm{~nm}$. 
The induced dipole seen in the cylinder and the sphere allows for the introduction of another important topic related to SERS enhancement. While this concept is for a single metal object, when there are two or more closely spaced objects there is the existence of coupled plasmon resonances. If there are two cylinders that are several diameters apart from one another, they can be considered two independent cylinders, however, as the cylinders get closer together, the field produced by each of their induced dipoles start to interact. The interaction changes the special configuration of the fields and shifts the energy of the resonances. The couple resonance comes from the interaction of the dipoles of each individual cylinder. Not only does the resonance shift but the local field intensity enhancement factor also increases between the two cylinders approach one another. ${ }^{31}$ These large enhancements are informally called hot spots. In many cases of SERS they provide enough enhancement to detect single molecules. ${ }^{31}$ The large increase in the local field intensity enhancement factor at the hot spots is normally associated with the spatial localization of the resonance at the gap. Figure 14 shows that the local field intensity enhancement factor is highly localized in the gap separating the two cylinders. When moving away from the center axis, and the hot spot, it is observed that the local field intensity enhancement factor greatly decreases. ${ }^{31}$ 

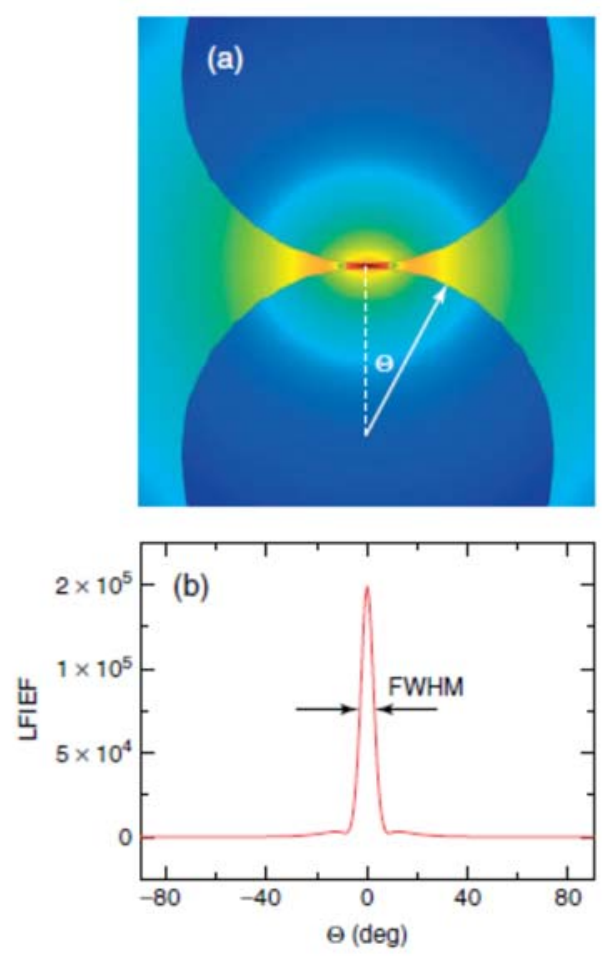

Figure 14: Formation of hot spots between two Ag cylinders. (a) Spatial distribution of the LFIEF between two Ag cylinders at $471 \mathrm{~nm}$, red is the most intense while blue is the weakest. Localized coupled plasmon resonance are the most intense. (b) the variation of the LFIEF on the surface of the cylinder as a function of $\theta^{31}$

The local field intensity enhancement factor is the increase in intensity that a molecule would experience at a specific point on the metal surface, therefore, all optical magnitudes that depend directly on the intensity are modified by the local field intensity enhancement factor (typically enhanced). Raman scattering involves the interaction of two photons, one from the incident electromagnetic radiation from the laser and another re-emitted by the molecule. If a molecule is placed on the surface of a metal where the laser field is enhanced through a local field intensity enhancement factor, more Raman processes will be produced. The local field intensity enhancement factor will increase 
$\omega_{L}$, the incident field, as though increasing the laser power. Therefore, more scattered photons will be observed at $\omega s$, the emitted field. If the molecule is in a position that enhances the emitted field at $\omega s$, the Raman intensity will also be increased because the emission is directly coupled to the excitation in the Raman process. Due to the two photon process, the Raman process benefits from both the excitation and emission enhancements, local field intensity enhancement factor at both $\omega_{L}$ and $\omega_{S}{ }^{25,31}$ Therefore the enhancement process for SERS can be written:

$$
E F=\operatorname{LFIEF}\left(\omega_{L}\right) x \operatorname{LFIEF}\left(\omega_{S}\right)
$$

Equation 7 is the simplest version of the SERS EF. The SERS EF at a given point can then be determined by combining Equations 2 and 7:

$$
E F \sim \operatorname{LFIEF}^{2}\left(\omega_{L}\right)=\frac{|E(r)|^{4}}{\left|E_{0}(r)\right|^{4}}
$$

Equation 8 shows the $|E|^{4}$ approximation for the SERS enhancement and can be an estimation of the SERS enhancements in a single molecule located at point $r$. In most experimental cases however, single molecules are not measured. Rather, many molecules are spread across different places on the metal surface and have very different EFs. Going back to Figure 14, the distribution of the local field intensity enhancement factor observed close to and far away from the hot spot shows how molecules that differ in position can have very different EFs. Therefore, surface-averaged SERS measurement are more appropriate to measure both the substrate performance as well as the SERS signal. $^{31,25}$ The metal particle, therefore is the main contributor to the SERS effect, not the molecule to be detected, though the molecule can have some effect and is related to the chemical enhancement that can be observed. 
Chemical enhancements operate independently of the electromagnetic mechanism previously discussed and are a much smaller contributor to the SERS effect than electromagnetic enhancements. These effects are related to the sample to be analyzed itself, such as molecular orientations and adsorptions. These processes can increase the SERS enhancement but are not related directly to the SERS mechanism itself. Chemical enhancements correspond to any modification of the Raman polarizability upon adsorption of the molecule onto the metal surface. ${ }^{25,35}$ The most common mechanism of modification in polarizability is through charge-transfer, where the modified polarizability is more resonant with the excitation than the original polarizability. This causes a natural increase in the Raman intensity due to the increase in resonance. Generally, chemical enhancement does not only appear for chemically bound molecules, it can also occur do to molecules being in close proximity with the metal surface. It is important to note that chemical enhancement can also have a negative effect on the SERS enhancement. For example, a negatively charged molecule cannot adsorb onto the surface of a negatively charge silver particle due to strong repulsions and no SERS signal will be produced. This is important when dealing with different types of molecules using a single optimized SERS method. ${ }^{25}$

\subsubsection{Improvement of enhancement through aggregation}

The largest SERS enhancements generally occur between two particles, where hot-spots form. The hot spots a result of the coupled resonances of two particles in close proximity, thus increasing the electromagnetic field which was discussed previously (Figure 14). In order to create these situations in colloidal solutions, these nano-particles must be aggregated to become useful SERS substrates. The colloidal solutions prepared 
should be stable solutions so that aggregation can be completely controlled by the addition of aggregating agents at an optimized concentration. ${ }^{25}$ Aggregation agent concentrations must be controlled in order to not exceed the critical coagulation concentration which causes particles to completely precipitate out of solution due to being highly aggregated. ${ }^{25}$

The most common types of aggregating agents are salts (active or passive). Passive salts are those that are not thought to interact strongly with the metals or other parts of the system, potassium nitrate $\left(\mathrm{KNO}_{3}\right)$ for examples. The addition of passive salts increases the ionic concentration in solution while decreasing the Coulomb interaction between the particles, thus producing aggregation. Active salts, such as potassium chloride $(\mathrm{KCl})$, change the ionic strength of the solution and can have additional interactions with other parts of the system, such as the metal substrate. These active salts, mainly chloride containing salts, are most commonly used for colloidal SERS experiments and are their interactions are well understood. ${ }^{25,36,37}$

The interaction of chloride $\left(\mathrm{Cl}^{-}\right)$ions producing an "activated" SERS signal is known as "chloride activation" and refers to the additional increase in enhancement observed due to the presence of chloride ions in addition to the creation of hotspots formed during aggregation alone. There is also additional anion enhancement as well. Chloride activation occurs by the displacement of the stabilizing agent on the surface of the metal nanoparticle by the chloride ions. Chloride activation changes the surface charge of the particles, increasing the negative charge, and changes the ionic strength of the solution. Chloride ions can also modify the adsorption properties of the SERS analyte, helping the adsorption of the analyte to the SERS surface. These ions can also 
increase the chemical enhancement property of SERS. ${ }^{25,37}$ While the anion-enhancement is well studied, the effect of the cation of the salt has only recently been shown to have an effect. It has been found that the smaller the cation (even with the same number of chloride ions) the larger the SERS enhancement. ${ }^{6,37}$

It is important to note that while SERS parameters can be generalized, the optimum conditions are highly variable. While some trends can be established, there is no simple relationship between colloids, aggregating agents, and analytes. This has been previously discussed in a number of optimization papers. ${ }^{36,37,38}$ When designing a SERS experiment, optimization has to be carried out between analyte and aggregating agent as well as with colloidal solutions used regardless of the similarity in analytes..$^{37,38}$

\subsubsection{Nanoparticle formation and properties}

The two main parameters in a SERS experiment (though there are many more) are the analyte and the metallic substrate. In order to produce the highest signal enhancement, the selection of the metallic substrate is critical. Generally, metal substrates that produce the strongest plasmon resonances (local field intensity enhancement factor) are selected. As discussed above, the plasmon resonance response of the substrate is the main facilitator of the SERS enhancement. The resonance is wavelength dependent, therefore, substrate selection should be determined based on the laser wavelength to be used. The wavelength selection does not affect the magnitude of Raman shifts, as Raman shifts are only dependent on the energy difference between the incident and emitted photon after interaction with the molecule. The same compound will display identical Raman shifts regardless of the laser wavelength. ${ }^{25,26}$ A good substrate at one wavelength may be very poor at another. Silver and gold are the main 
metals used in SERS experiments due to sustaining plasmon resonances in the visible/near-IR range used in Raman systems observed in Figure 13 of the previous section. $^{25}$ If silver is used as the SERS substrate, an ideal wavelength would be at approximately $350 \mathrm{~nm}$, this is where Ag produces a maximum local field intensity enhancement factor with a very narrow range for a spherical particle. If gold is selected as the SERS substrate for a spherical particle, a wider range of laser wavelengths can be used due to the broad local field intensity enhancement factor from approximately 500 $850 \mathrm{~nm}$. Gold produces a maximum enhancement when the wavelength selected is approximately $550 \mathrm{~nm}$, however, this maximum is well below that of $\mathrm{Ag}$ at its optimal wavelength. ${ }^{31}$

Silver and gold colloidal solutions, are produced by reduction of the metal containing salt by a number of methods. ${ }^{39,36}$ Colloidal solutions are advantageous over solid support SERS substrates due to their quick, reproducible synthesis, as well as having the capability to provide a fresh substrate to perform each subsequent analysis. There is no further complication of removing an analyte from a substrate. The colloids exist in solution due to stabilization either by columbic repulsions among the particles or by the introduction of a stabilizing agent which coats the surface of the particles and prevents aggregation. ${ }^{25}$ In some cases, the reducing agent can also serve as the stabilizer. ${ }^{39}$ In general, gold particles are more easily stabilized then silver over longer periods of time (greater than a year). Citrate-reduced colloids are the most commonly generated nanoparticles for SERS analysis.

Citrate-reduced colloids are commonly referred to as "Lee-Meisel" colloids because of their work providing the initial synthesis method. ${ }^{39}$ The colloids are prepared 
by bringing a solution of silver nitrate $\left(\mathrm{AgNO}_{3}\right)$ or chloroauric acid $\left(\mathrm{HAuCl}_{4}\right)$ to boiling, adding a $1 \%$ by weight sodium citrate solution, which acts as the reducer and stabilizer, and refluxing for an hour and bringing to room temperature. ${ }^{39}$ The procedure produces a silver solution with a grey-yellow color and a UV/Vis absorption maximum between $400-430 \mathrm{~nm}$ and a gold solution with a wine red color and a UV/Vis absorption maximum between $520-530 \mathrm{~nm} .{ }^{25,39}$ Other reducing agents have been applied, in particular to the synthesis of silver nanoparticles. The most common of which is sodium borohydride, however, these particles are not as stable as sodium citrate reduced colloinds. ${ }^{25,36,40}$ In all these methods of synthesis, the formation of the particles is not completely understood. It is proposed that colloid formation begins by a nucleation process, which then triggers the growth of the particle to a certain size and shape. This can be controlled by limiting the amount of metal or reducing agent present, thus stopping the reaction or by changing the temperature of the reaction quickly. ${ }^{25}$

Once colloidal solutions are synthesized they can be characterized in a number of ways. The first, most simply is using scanning electron microscopy (SEM) to evaluate the size and shape of the particles. The SEM analysis can be performed on colloidal solutions by first drying the solution onto the appropriate substrate and taking an image of the now clustered particles. The SEM gives a look at the basic shape and size of the particles individually but does not provide information about the whole solution or how the particles appear in the whole solution. To obtain information on the entire solution, optical or light-scattering methods such as UV/Vis spectroscopy are often employed. ${ }^{25}$

Ultra-violet/visible spectroscopy provides additional information about the colloidal substrate. The UV/Vis absorption maximum of colloidal solutions, is associated 
with a resonant optical response at the wavelength which corresponds to the localized surface plasmon resonance. Due to SERS occurring as a result of the interaction with the localized surface plasmon resonance, when the incident laser is at the same wavelength as observed for the UV/Vis absorption maximum, the largest SERS enhancement is expected. ${ }^{25}$ These measurements can also be used for quick "qualitycontrol" of different batches of particles as it is dependent on the shape but not the size of the particles. ${ }^{25}$

Dynamic light scattering (DLS) is used to measure the size distribution of colloids in solution. The DLS technique relies on the measurement of intensity fluctuations in the light scattered by a monochromatic source. The movement of the colloids (Brownian motion) is directly related to these fluctuations and the particle size can be indirectly determined by estimating the diffusion coefficient from the intensity fluctuations. The DLS technique is widely used as a quick characterization technique for the size and dispersion of colloidal solutions. ${ }^{25}$

\subsection{SERS for Drug Detection}

Surface enhanced Raman detection has been applied to a number of different drugs of abuse, either as standards or after a separation technique. In previous work by Cîntã et al., the analysis of diazepam and nitrazepam was performed on silver colloid surfaces in aqueous solution. ${ }^{40}$ The nanoparticles were synthesized using borohydride reduction. The drugs were detected and identified at a concentration of approximately 30 $\mathrm{ng} / \mathrm{mL}$ with excitation wavelength of $488 \mathrm{~nm}$. Though the structures of these two compounds are very similar, they can be easily distinguish on the basis of differences in Raman vibrational frequencies. ${ }^{40}$ 
Surface enhanced Raman spectroscopy has been employed for the analysis of trace identification of some controlled substances. ${ }^{41,42}$ Silver nanoparticles were used to detect morphine, codeine and hydrocodone in trace quantities with $\mathrm{NaCl}$ aggregation. ${ }^{41}$ Nanoparticles, aggregating agent, and sample were applied directly to microscope slides for analysis. The spectra produced provided sufficient spectral information to differentiate these three structurally similar molecules as well as providing information on to how the molecules interact with the nanoparticles. Detection limits were not determined and spectra were produced with the addition of $1 \mu \mathrm{g}$ of drug of interest. Fox et. al., combined electrostatic lifting with SERS analysis. ${ }^{42}$ Ultra-trace analysis was performed by the electrostatic lifting of drug residues from mixtures of drug in sand ( $15 \%$ by weight) directly onto gold plated Mylar lift films. Surface enhanced Raman spectroscopy was then performed on the films. This method shows the applicability of SERS to trace level drug detection. ${ }^{42}$

Surface enhanced Raman spectroscopy has also been applied in the analyses of other drugs of abuse, such as sulfa drugs, amphetamines, cocaine, and heroin. ${ }^{43,44,45,46,47}$ Sutherland et al. were able to detect the sulfa drugs sulfadiazine, sulfamerazine, and sulfamethazine by SERS using silver colloidal dispersions. These dispersions were prepared using the common sodium borohydride reduction method with no addition of any aggregating agent. They found detection limits below $10 \mathrm{ng} / \mathrm{mL} .{ }^{46}$

Other types of drugs mentioned above were analyzed by applying the drug sample directly onto a solid planar SERS substrate either directly or following a liquid separation technique rather than using colloidal solutions. Bell and colleagues have performed a number of studies describing the analysis of solid tablet samples amphetamines and 
amphetamine derivatives by Raman spectroscopy and SERS. ${ }^{48,49,50,51,52}$ Using SERS, the group was able to improve the detection of low levels of amphetamines. For example, they were able to observe a concentration of $38 \mu \mathrm{g} / \mathrm{mL}$ in the detection of $2,5,-$ dimethoxy-4-bromoamphetamine (DOB) in lactose incipient, which is well below the concentrations commonly seen in illicit tablets (10 mg per tablet). The preferential enhancement was due to the selective adsorption of the DOB to the silver colloids over that of the lactose, enhancing the Raman spectra of the DOB to overwhelm that of the lactose. ${ }^{52}$ The selective enhancement can be applied to complex matrixes by using a substrate where drugs of interest are preferentially absorbed onto the substrate surface over the solvent and other components of the matrix.

Surface enhanced Raman spectroscopy has also been applied to the analysis of drugs following a liquid separation technique, such as high performance liquid chromatography (HPLC) or capillary electrophoresis (CE). ${ }^{43,44,45,53,54}$ Often testing for the presence of drugs involves an analysis in physiological fluids. In such situations, the SERS analysis would be complicated by the presence of interferences such as proteins, therefore, the small quantity of drug present must be extracted and cleaned up prior to using SERS detection. ${ }^{43,44,45}$ Cleanup can also include preconcentration of the sample to improve the sensitivity of the analysis. HPLC has been utilized as a separation technique prior to SERS for the analysis of cocaine, heroin, amphetamines and 1,4-benzodiazepines by Schneider and coworkers. ${ }^{43,44,45}$ Each study shows that following a liquid-liquid extraction from biological fluids, HPLC can separate a sufficient amount of material for SERS off-line detection $(1 \mu \mathrm{g} / \mathrm{mL})$. The choice of eluent is extremely important due to competition with the analyte for adsorption to the SERS substrate. Many organic eluents, 
such as acetonitrile, compete with the analyte for the SERS substrate. However, other buffers and eluents such as phosphate and methanol, do not compete for the SERS substrate and in fact, the increasing salt content can enhance the SERS signal. In these investigations, the eluent was sprayed onto a substrate followed by SERS analysis. The substrates were typically colloidal gold or silver deposited onto the surface of a microtiter plate or roughened glass microscope slide.

Capillary electrophoresis/SERS has also been developed with at-line coupling similar to those methods performed by HPLC. ${ }^{54}$ In the case of CE, the run buffer was a formic acid solution with no organic modifier. Dyes were detected using a UV detector and then deposited on a substrate following elution from the CE column. The main benefit of using $\mathrm{CE}$ as a separation technique over HPLC is the ability to use aqueous eluents instead of aqueous/organic mixtures. Removing the organic solvents provides less SERS interference and adsorption competition.

Another separation technique that has been used with SERS is solid phase extraction followed by elution of drugs of interest into sol gel filled capillaries that were doped with gold and silver particles. ${ }^{55}$ This study was able to detect eighty drugs of abuse, including a number of benzodiazepines and other DFSA drugs, from simulated saliva samples, to a detection limit of $50 \mathrm{ng} / \mathrm{mL}$ for cocaine. Five other drugs were tested, of them, diazepam was detected at a limit of $1000 \mathrm{ng} / \mathrm{mL}$, which is well above required detection limit for this type of drug. In this method, analytes were dried in the sol gel filled capillaries, rather than analyzed as liquids. ${ }^{55}$ 


\section{GOALS AND OBJECTIVES}

The goal of this thesis was to produce a surface enhanced Raman procedure capable of providing sensitive detection of benzodiazepines from biological samples as a rapid and universal alternative to traditional immunoassays. The procedure would permit drug speciation, making it particularly useful in situations such as DFSA where quick determination of the unknown chemical agent is important. Such procedures should require minimal sample input and be sufficiently rapid and sensitive to permit use in point of care situations.

The study includes three main objectives. The first objective was to optimize the detection of benzodiazepines on SERS substrates. This included the development of optimal SERS metal substrate as well as the determination of best possible aggregating agent to permit easy coupling of Raman signal to the analyte. The second part of the study was to investigate possible interferences commonly observed in urine samples of drug facilitated sexual assault and to determine the extent to which these compounds interfered with the SERS analysis of benzodiazepines. The final part of the study was to validate the method and determine an extraction technique for use with simulated toxicological samples. The overall procedure was validated using a panel of benzodiazepines implicated in drug facilitated sexual assault. The validation process included spectral analysis of the Raman shifts for individual benzodiazepines, determination of analytical figures of merit, and analysis of simulated/spiked toxicological samples. 


\section{DETERMINATION OF SURFACE ENHANCED SUBSTRATE}

\subsection{Introduction}

There are a wide variety of possible surface enhanced Raman active substrates that can be used in the development of an optimized SERS system. To simplify the optimization, only the most commonly used metals, silver (Ag) and gold (Au), due to

their optimum optical properties in the laser range applicable for SERS, were examined. ${ }^{25}$ The goal of the overall method is to provide a quick screening technique for benzodiazepines in urine samples and due to the lack of interference in the SERS spectrum by water, colloidal solutions will provide a quick, inexpensive, disposable SERS substrate to provide analysis of such aqueous solutions as urine.

A number of methods have been described for the synthesis of gold and silver spherical nanoparticles. The most common of which is the Lee Meisel method involving the reduction of the metal containing salt with sodium citrate. ${ }^{39}$ Other methods involving reduction of metal salts involve using sodium borohydride or hydroxylamine hydrochloride as the reducer. ${ }^{36,56}$ In order to assess the quality of the particles in relation to benzodiazepines, chlordiazepoxide was used due to its solubility in water, thus not introducing any possible interfering organic solvents. Aggregating agents of magnesium chloride were prepared at a commonly reported concentration. ${ }^{36}$ Chlordiazepoxide $\mathrm{HCl}$ was also prepared in varying concentrations of methanol in order to determine the maximum concentration of methanol that can be used for dissolving other benzodiazepines without interfering the their SERS spectra. 


\subsection{Experimental}

All solutions were prepared with ultrapure water. Glassware was cleaned with detergent followed by deionized water and then methanol.

\subsubsection{Nanoparticle Synthesis}

Three methods of synthesis were performed for silver nanoparticles while only one was prepared for gold. The first silver synthesis (AgI) is based on the Lee Meisel method. Two $\mathrm{mL}$ of a $1 \%$ trisodium citrate solution was added to $100 \mathrm{~mL}$ of $1 \mathrm{mM}$ $\mathrm{AgNO}_{3}$ was heated to boiling. Boiling was maintained for one hour under reflux then cooled to room temperature and brought to a volume of $200 \mathrm{~mL}$ with deionized water producing a dark grey solution with a relative concentration of $0.2 \mathrm{mM}$ silver. ${ }^{39,36}$ The gold colloidal solution was also based on the Lee Meisel method. Two mL of a 1\% trisodium citrate solution was added to $50 \mathrm{~mL}$ of $0.5 \mathrm{mM} \mathrm{HAuCl}_{4}$ which was heated to boiling. Boiling was maintained for thirty minutes under reflux then cooled to room temperature and diluted to $50 \mathrm{~mL}$ with deionized water producing a clear, dark red solution. ${ }^{39,36}$

The second silver synthesis (AgII) is based on the Creighton method. Twenty five $\mathrm{mL}$ of a $1 \mathrm{mM} \mathrm{AgNO}_{3}$ solution was added in $5 \mathrm{~mL}$ aliquots to $75 \mathrm{~mL}$ of an ice cooled $2 \mathrm{mM} \mathrm{NaBH}_{4}$ solution with vigorous stirring. After the addition of all the silver nitrate solution stirring was continued for an additional 10 minutes. Twenty five $\mathrm{mL}$ of deionized water was added to the resulting solution producing a brown solution with a relative concentration of $0.2 \mathrm{mM}$ silver. ${ }^{57,36}$ The third silver synthesis (AgIII) is based on the Leopold Lendl method. Four and a half $\mathrm{mL}$ of $0.1 \mathrm{M} \mathrm{NaOH}$ was mixed with $5 \mathrm{~mL}$ of a $60 \mathrm{mM}$ solution of hydroxylamine hydrochloride. The resulting solution was added 
rapidly to $90 \mathrm{~mL}$ of vigorously stirred $1.1 \mathrm{mM} \mathrm{AgNO}_{3}$ and stirring was continued for 10 minutes until a milky grey solution was produced. To this resulting colloid solution, 395 $\mathrm{mL}$ of deionized water was added to produce a relative concentration of $0.2 \mathrm{mM}$ silver. ${ }^{56}$

Particles were characterized by using three main analytical techniques. The ultraviolet-visible absorbance (Varian Cary 100 Bio UV-visible spectrophotometer) was measured to determine relative size and optimum incident laser. Colloidal solutions were added directly to quartz cuvettes and measured. Physically particle size was measured using scanning electron microscopy (JEOL JSM 5900LV, $3.0 \mathrm{~nm}$ resolution). Colloidal solutions were centrifuged for 10 minutes and supernatant was removed and particles were dried overnight. The resulting pellets were applied to carbon studs for SEM imaging. Dynamic light scattering was performed on gold nanoparticles for further size verification with three runs each with 16 scans.

\subsubsection{Sample Preparation}

Magnesium chloride at a concentration of $1.67 \mathrm{M}$ was prepared in water for use as a sample aggregating agent. Aggregating agents were further analyzed in Chapter 4. Chlordiazepoxide $\mathrm{HCl}$ dilutions were prepared in deionized water from $50-50,000$ $\mathrm{ng} / \mathrm{mL}$. Chlordiazepoxide $\mathrm{HCl}$ was also prepared at a concentration of $25,000 \mathrm{ng} / \mathrm{mL}$ in varying concentration of methanol $(1 \%-10 \%)$ in water.

The SERS spectra were obtained with a Perkin Elmer Raman Station 400F with $785 \mathrm{~nm}$ excitation for four spectral scans each at a 10 second exposure. The power at the sample surface was $100 \mathrm{~mW}$. Samples as prepared below were transferred to SERS inactive quartz cuvettes for analysis. For SERS experiments, $980 \mu \mathrm{L}$ of gold colloids were placed in sample vial followed by $10 \mu \mathrm{L}$ of the aggregating agent. This was 
vortexed for 30 seconds. Ten $\mu \mathrm{L}$ of sample solution was then added and vortexed for 30 seconds. ${ }^{36}$

\subsection{Results and Discussion}

\subsubsection{Size characterization of particles}

All particles observed were spherical in shape with a range of sizes $(20-30 \mathrm{~nm})$ visible in the SEM micrographs.

AgI

This synthesis method, sodium citrate reduction, produced a dark grey solution. The UV-visible absorbance showed a maximum wavelength at $347 \mathrm{~nm}$ and the SEM micrograph measured particle sizes consistent with approximately $20 \mathrm{~nm}$. Figure 15 shows these results.
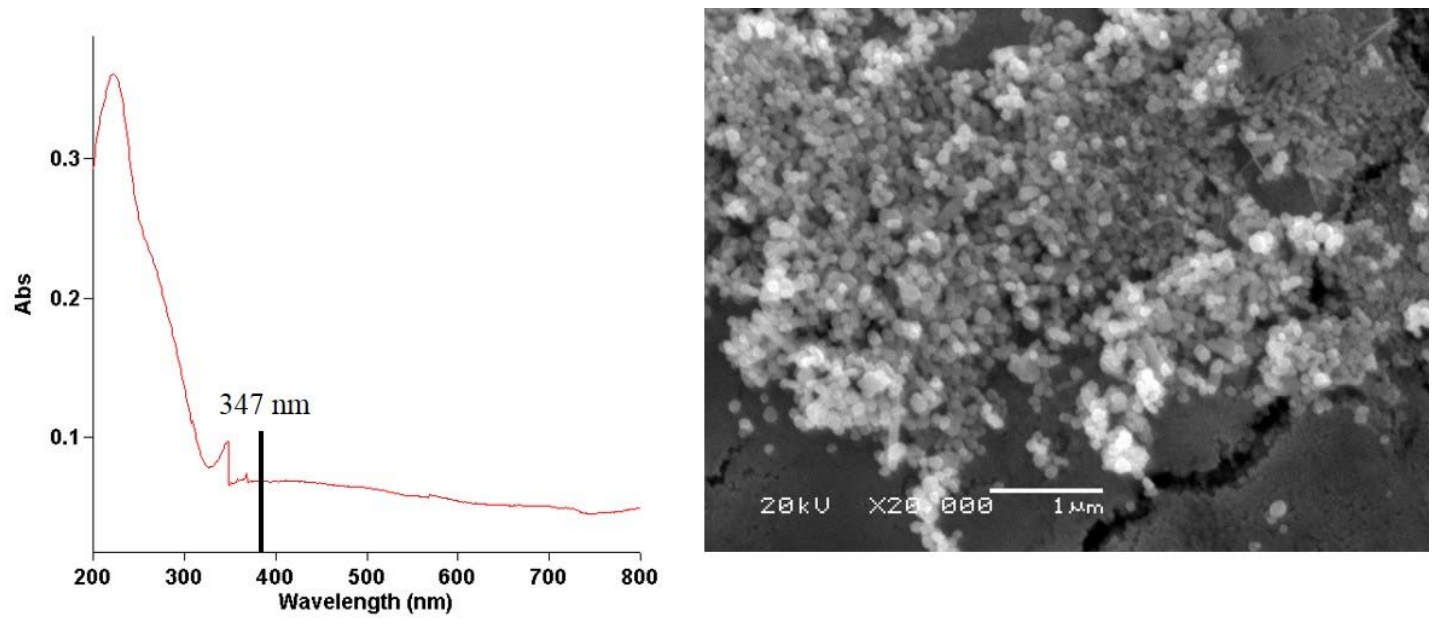

Figure 15: UV-visible absorbance and SEM micrograph of AgI particles

AgII

The second synthesis method, sodium borohydride reduction, produced a brown solution. The UV-visible absorbance showed a maximum wavelength at $393 \mathrm{~nm}$ and the 
SEM micrograph measured particle sizes consistent with approximately $20 \mathrm{~nm}$. Figure 16 shows these results.
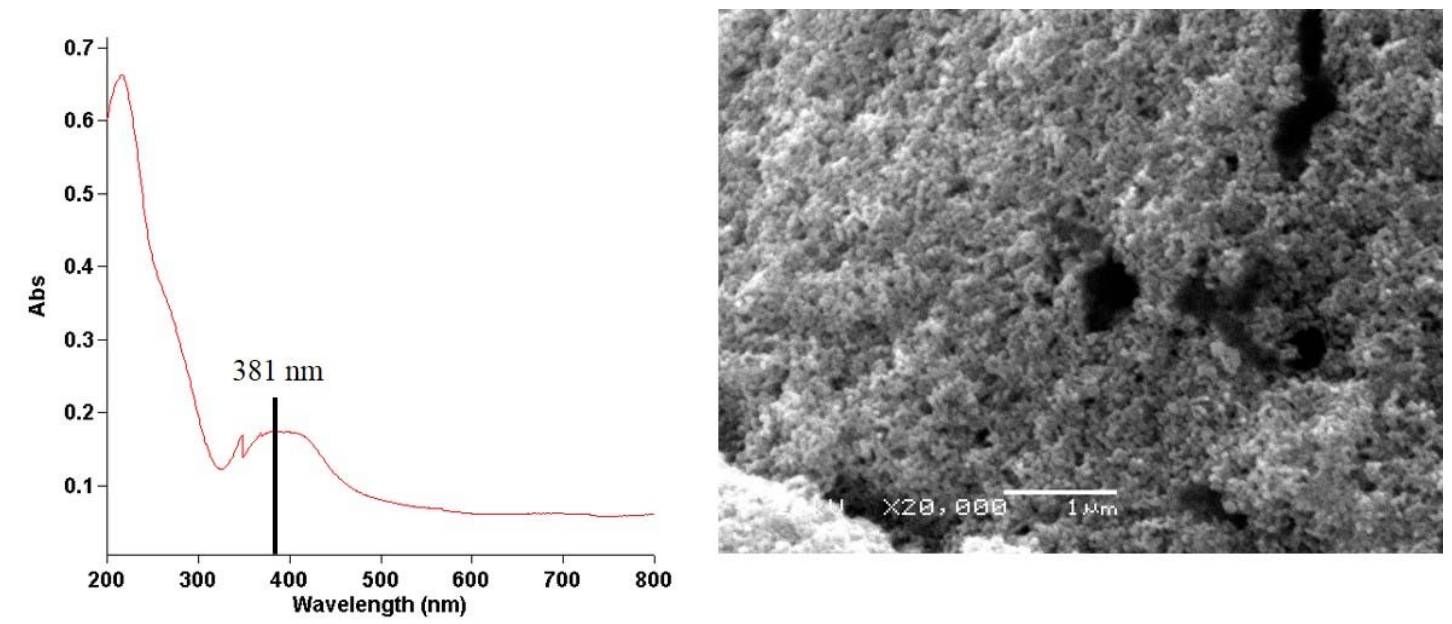

Figure 16: UV-visible absorbance and SEM micrograph of AgII particles

AgIII

The third synthesis method, hydroxylamine hydrochloride reduction, produced a milky grey solution. The UV-visible absorbance showed a broad absorbance band with maximum wavelength at $425 \mathrm{~nm}$ and the SEM micrograph measured particle sizes consistent with approximately $20 \mathrm{~nm}$. Figure 17 shows these results. 

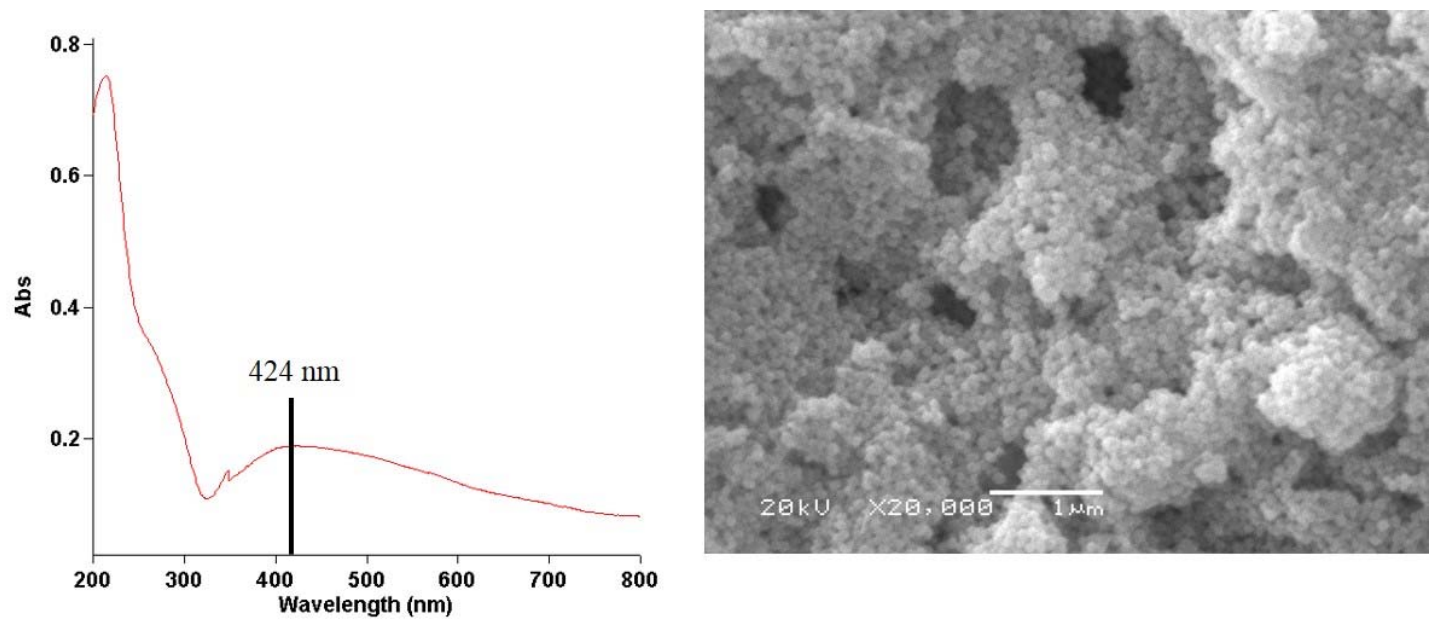

Figure 17: UV-visible absorbance and SEM micrograph of AgIII particles

$A u$

The gold synthesis method, a sodium citrate reduction, produced a clear dark red solution. UV-visible absorbance showed a maximum wavelength at $522 \mathrm{~nm}$ and the SEM micrograph measured particle sizes consistent with approximately $30 \mathrm{~nm}$. Figure 18 shows these results.
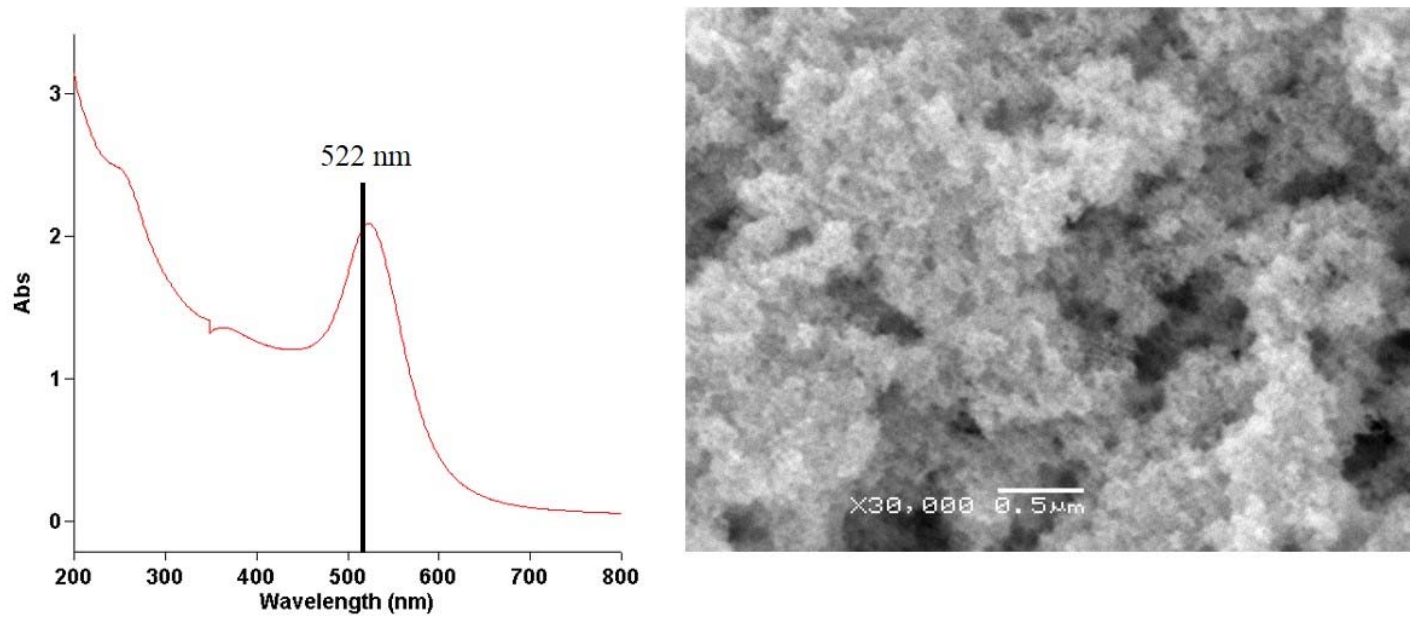

Figure 18: UV-visible absorbance and SEM micrograph of gold particles 
In addition, dynamic light scattering was performed on these gold nanoparticles. Figure 19 shows the size distribution curve of the particles, ranging from $10-80 \mathrm{~nm}$ in size with an average particle size of $28 \mathrm{~nm}$ in diameter.

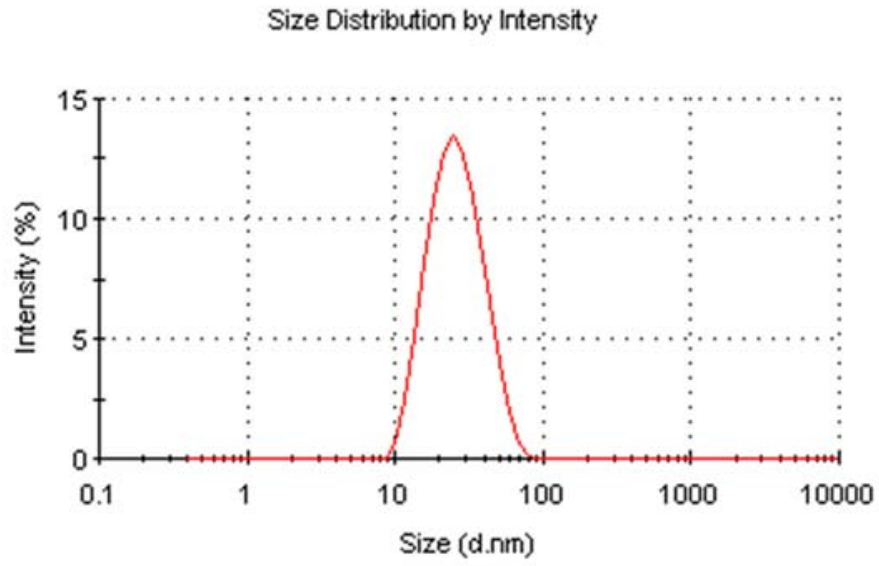

Figure 19: DLS size distribution of gold nanoparticles; particles ranged in size from 10$80 \mathrm{~nm}$ with an average particle size of $28 \mathrm{~nm}$

\subsubsection{Surface enhanced Raman validation}

First it was determined whether these particles would produce any extraneous spectra that would have any interference with the drugs to be analyzed in further studies. Water was added to the particles and the aggregating agent as described in the methods and the SERS spectra were obtained. These blanks produced flat baselines with a large peak at $200 \mathrm{~cm}^{-1}$ due to water, however this peak does not interfere in the spectral range necessary for drug analysis $\left(1800-400 \mathrm{~cm}^{-1}\right)$.

Chlordiazepoxide $\mathrm{HCl}$ was used as a model compound for the optimization of nanoparticles for their use in SERS. The compound is water soluble and does not require potentially interfering organic solvents for dissolution. Samples of chlordiazepoxide 
were added to prepared nanoparticles using $\mathrm{MgCl}_{2}$ as an aggregating agent as described in the methods.

The three silver particles all produced similar spectra for chlordiazepoxide with varying intensities under the same conditions. The gold particles, however, produced a Raman spectra that was significantly different that those produced with silver particles, Figure 20. This is likely due to different interactions between the drug and the particles. As a result of the much reduced number of peaks in the silver spectrums, it is likely the drug is binding the surface of the silver particles while only being in close proximity to the surface of the gold particles. ${ }^{25}$

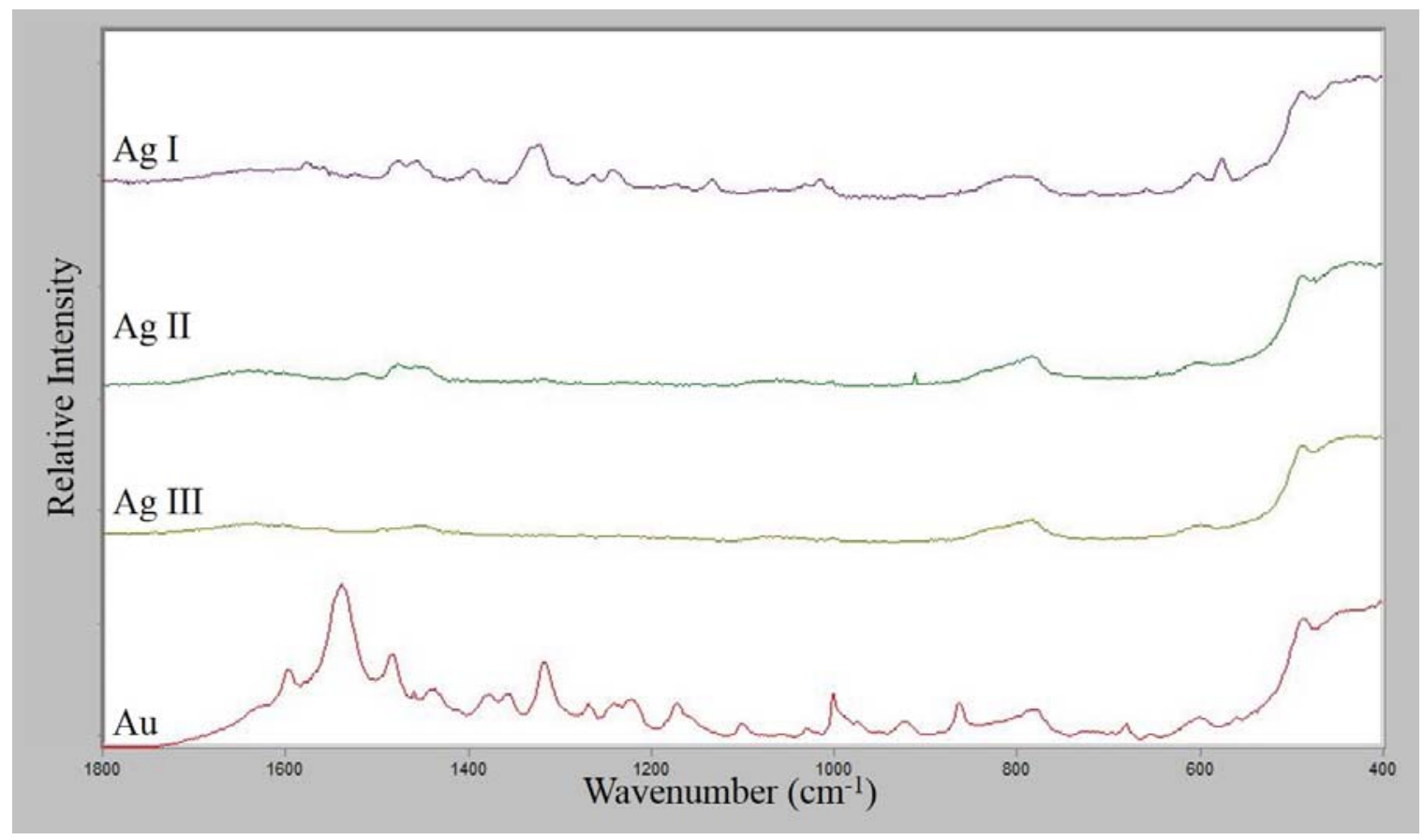

Figure 20: SERS comparison of chlordiazepoxide between four synthesized particles.

While some spectral information is observed for Ag I particles, Au provided the highest intensity peaks as well as more spectral information for chlordiazepoxide when compared to the Ag particles. 
Limits of visible detection were also examined to determine whether SERS spectra could be obtained below the high concentrations prepared. In all cases the silver nanoparticles did not produces a SERS spectra for chlordiazepoxide at any concentration prepared below 50,000 ng/mL. On the other hand, the gold particles produced spectra at all concentrations tested, down to $50 \mathrm{ng} / \mathrm{mL}$. The overall goal of this technique is to be able to detect benzodiazepines at a concentration of $10 \mathrm{ng} / \mathrm{mL}$ or below. Therefore, the gold nanoparticles were determined to be the optimum colloids for the analysis of benzodiazepines and were used in the following study.

\subsubsection{Methanol Study}

Chlordiazepoxide $\mathrm{HCl}$ was prepared at varying concentration of methanol (1\% $10 \%)$ as well as solutions of methanol at the same concentration without any drug in water. The SERS spectra were obtained for all samples. Figure 21 shows the SERS spectra of pure methanol, $10 \%$ methanol in water and $25,000 \mathrm{ng} / \mathrm{mL}$ chlordiazepoxide $\mathrm{HCl}$ in $10 \%$ methanol. 


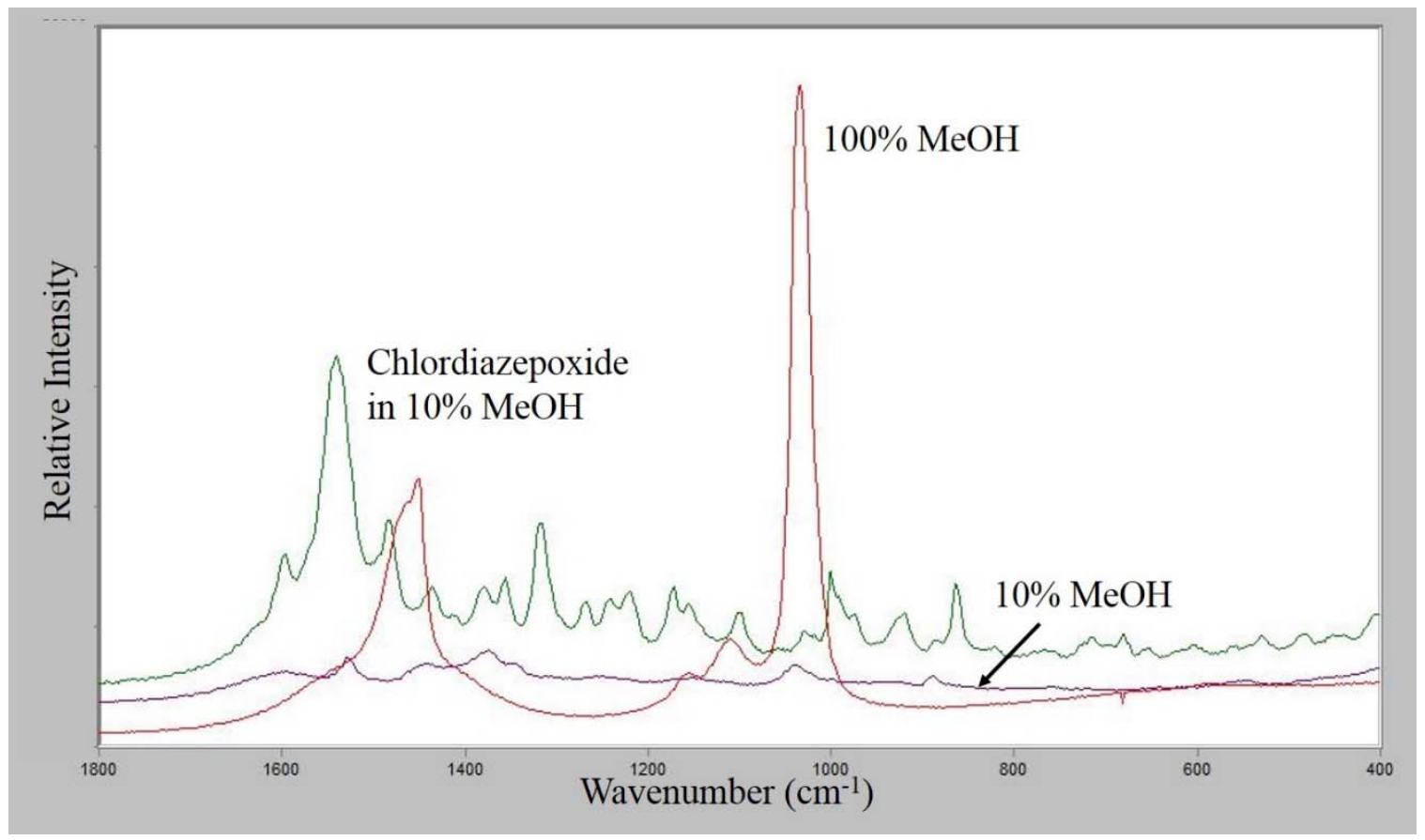

Figure 21: Comparison of methanol spectra with chlordiazepoxide $\mathrm{HCl}$ in $10 \%$ methanol. At $10 \%$ methanol with the drug, any slight methanol peaks are completely suppressed by the drug.

It was found that while methanol concentrations without the addition of drug did provide very slight methanol spectra at a concentration of $10 \%$ methanol, no methanol peaks were observed in the SERS spectra of chlordiazepoxide $\mathrm{HCl}$ up to a concentration of $10 \%$. Due to this, all subsequent analyses of benzodiazepines were prepared in $10 \%$ methanol in water as it provides sufficient organic solvent to facilitate dissolving the drug and yet is not an interference in the SERS spectra of these drugs.

\subsection{Conclusions}

It was determined that the optimum SERS substrate for the analysis of benzodiazepines are spherical gold colloidal solutions prepared by a modified Lee Meisel method. This is due to their ability to provide spectra of chlordiazepoxide, the model 
benzodiazepine used, at the minimum concentration prepared here, $50 \mathrm{ng} / \mathrm{mL}$. The intensity of these peaks were still well above the baseline, therefore, the Au particles should allow for detection of benzodiazepines below the $50 \mathrm{ng} / \mathrm{mL}$ concentration prepared here. All three Ag particles prepared could not provide spectra at the $50 \mathrm{ng} / \mathrm{mL}$ concentration prepared here, making these Ag particles not useful for analysis of benzodiazepines. This issue is due to the wavelength of the laser in use, $785 \mathrm{~nm}$. As discussed in Chapter 1.3.2, the SERS mechanism relies heavily on the local field intensity enhancement factor (LFIEF) experienced by a molecule on the metal surface which is dependent on the wavelength of incident energy. Figure 13 shows that for silver, the highest local field intensity enhancement factor on spherical particles occurs in a very narrow wavelength range with a maximum local field intensity enhancement factor at a wavelength of $\sim 350 \mathrm{~nm}$. At $785 \mathrm{~nm}$, the local field intensity enhancement factor is close to zero. On the other hand, gold produces a high local field intensity enhancement factor over a very wide wavelength range with a maximum at $\sim 550 \mathrm{~nm}$. At $785 \mathrm{~nm}$, the local field intensity enhancement factor is approximately $11 .{ }^{31}$ Here, we have shown that experimentally at an incident wavelength of $785 \mathrm{~nm}$, gold and silver follow the theory and $\mathrm{Au}$ is the better substrate.

It was also determined that benzodiazepines can be dissolved in a solution up to $10 \%$ methanol without interference. When dissolved in a concentration about $10 \%$ methanol, the spectral peaks from the methanol begin to invade the SERS spectra of the drug and present a problem. These parameters were used in all remaining studies. While the silver particles prepared did produce some enhancement, the observable limits of 
detection are well above those that are need to use this technique in the analysis of drug facilitated sexual assault samples $(10 \mathrm{ng} / \mathrm{mL})$. 


\section{COMPARISON OF AGGREGATIGN AGENTS FOR THE SURFACE- ENHANCED RAMAN ANALYSIS OF BENZODIAZPINES}

Doctor, E.L.; McCord, B., Comparison of aggregating agent for the surface-enhanced Raman analysis of benzodiazepines. Analyst 2013, 138 (20), 5926-5932 - Reproduced with permission of The Royal Society of Chemistry. ${ }^{6}$

\subsection{Abstract}

Benzodiazepines are among the most prescribed compounds and are commonly present in many toxicological screens. They are also of concern forensically in cases of drug facilitated sexual assault. Currently these compounds are predominantly analyzed using immunoassay techniques; however more specific screening methods are needed. This paper demonstrates the applicability of surface enhanced Raman spectroscopy as a method for the analysis and detection of benzodiazepines. The procedure involves mixing urine extracts with gold nanoparticles and appropriate aggregating agents for trace detection of these compounds and their metabolites. In this paper we will discuss the optimization of various parameters of this technique as well as its application to screening urine samples.

Eleven different benzodiazepines and metabolites were examined, including 1,2triazolo-benzodiazepines and 1,4-benzodiazpines. Experiments were performed using four different chloride salts, $\mathrm{MgCl}_{2}, \mathrm{CaCl}_{2}, \mathrm{KCl}$, and $\mathrm{NaCl}$, as aggregating agents for the colloidal gold nanoparticles. Overall it was found that each aggregating agent produced different levels of signal enhancement for each drug. $\mathrm{MgCl}_{2}$ provided the lowest limit of detection at $2.5 \mathrm{ng} / \mathrm{mL}$, and linearity over a wide range of concentrations for a variety of drugs chosen. It was also determined that the optimum $\mathrm{MgCl}_{2}$ concentration was $1.67 \mathrm{M}$ 
This method has shown the applicability of SERS for the detection of trace quantities of benzodiazepines in aqueous solutions as well as the optimization of the technique over a wide range of compounds. This technique can be utilized in the detection of trace benzodiazepines in toxicological samples following extraction of the analyte.

\subsection{Introduction}

Raman spectroscopy's use as an analytical technique in forensic science laboratories has increased in recent years. The technique utilizes an intense laser to induce dipole moments in selected molecules. Inelastically scattered light from the laser is measured as a shift in frequency from the incident beam frequency and the resulting intensity profile produces the Raman spectrum. ${ }^{25}$ Raman spectroscopy offers some analytical advantages over other common spectroscopic techniques. Powder samples can be analyzed directly without sample preparation..$^{58,49,59,60,61,62}$ This has been shown to provide rich spectral information that can distinguish compounds with closely related structures. ${ }^{60,61,62}$ Raman instrumentation can also be coupled with an optical microscope to study small particles. ${ }^{58,49,59}$ Lastly, unlike infrared spectroscopy where water is a major interference in the spectral analysis, samples can be run in an aqueous environment. ${ }^{43}$ Conventional Raman spectroscopy is not without its drawbacks. Fluorescence can obscure the spectrum of the analyte and unless the analyte is at high concentrations, the Raman signal is often very weak, limiting the trace detection of drugs. Surface-enhanced Raman spectroscopy (SERS) overcomes the limitations by providing an enhanced signal several orders of magnitude over conventional Raman spectroscopy and quenching fluorescence. ${ }^{25}$ 
SERS requires the use of a SERS active metal substrate, commonly gold, silver, or copper, such as colloidal metal particles in aqueous solutions or roughened metal films. The interaction of the analyte with the substrate causes an increase in signal intensity. Colloidal solutions are advantageous due to their simple synthesis in batches and relatively low cost. They also provide a fresh surface for each analysis preventing contamination between samples. ${ }^{25}$ To further increase the enhancement of the SERS signal, aggregate solutions are used. These agents are salt solutions which cause the nanoparticles to amass and form hot-spots which increase the signal intensity. Chlorine salts generally provide the greatest enhancement for two reasons. The chlorine ions displace the stabilizing agent to cause aggregation and they affect the ionic strength of the surrounding solution changing the surface charge of the substrate, therefore increasing the signal intensity. ${ }^{25}$

The technique of SERS has been examined for use in the detection of controlled substances such as for narcotics, amphetamines, nicotine, and benzodiazepine. . $3,47,45,44,50,51,48,49,41,52-54,63,41$ Often this analysis is done with dried drug samples, where the controlled substance is dried onto the SERS active substrate. However, SERS has the potential for use in aqueous solutions which can be adapted for the detection of these controlled substances in toxicological samples. SERS detection of diazepam and nitrazepam was performed on silver colloid surfaces in aqueous solutions at a concentration of $30 \mathrm{ng} / \mathrm{mL}^{40}$

Toxicology laboratories are inundated with drug-facilitated sexual assault cases that require quick screening for over 40 different possible compounds. There has been a significant increase in the prevalence of benzodiazepines in these types of case 
submissions. Benzodiazepines are a class of controlled substances that are commonly prescribed medication for anti-depression and anti-anxiety. The effects these compounds have on the central nervous system such as amnesia, drowsiness, impaired coordination, and confusion have resulted in their increasing use in the commission of drug-facilitated sexual assault. The most common procedure used for the initial detection of these compounds is the immunoassay, however, this technique has many disadvantages when used in the determination of benzodiazepines. First, in many commercially available immunoassays, including enzyme multiplied immunoassay technique II, kinetic interaction of microparticle in solution and radioimmunoassay, the cutoff levels for benzodiazepines fall between 100 and $300 \mathrm{ng} / \mathrm{mL} \cdot{ }^{19,20}$ While these levels may be consistent with overdose or therapeutic levels, in cases where benzodiazepines are used during drug facilitated sexual assault, the concentrations observed are much lower (50 $\mathrm{ng} / \mathrm{mL}$ ), and immunoassays may produce a false negative for the presence of benzodiazepines. Secondly, some benzodiazepines have a poor cross-reactivity with immunoassay systems, such as lorazepam. ${ }^{19,20}$ Due to these problems, many laboratories also employ GC-MS screenings for benzodiazepines. Sample preparation for the GC-MS of the benzodiazepines involves not only extraction of the drugs from the biological matrix but also for some benzodiazepines, derivatization is necessary in order to produce volatile compounds, further complicating the procedure. ${ }^{19,64}$

In this study, eleven different benzodiazepines were examined from two of the main classes of benzodiazepines, 1,2-triazolo-benzodiazepines and the 1,4benzodiazpines, as well as a number of metabolites. Their base structures can be seen in Fig. 22 while substituents for individual drugs are seen in Table 2. 

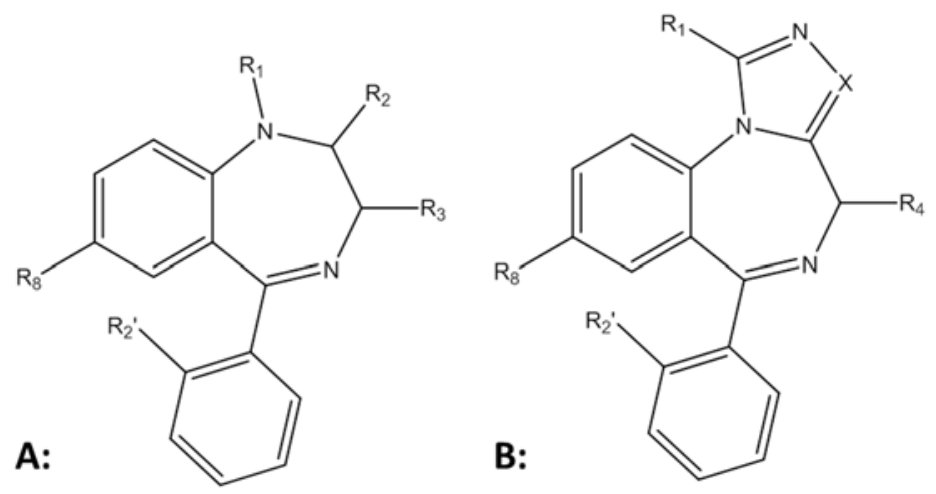

Figure 22: Base structures of 1,4-benzodiazepines (A) and 1,2-triazolo-benzodiazepines (B)

\begin{tabular}{|l|c|c|c|c|c|}
\hline \multicolumn{1}{|c|}{ 1,2- Triazolo- benzodiazepines } & $\mathbf{R}_{\mathbf{1}}$ & $\mathbf{X}$ & $\mathbf{R}_{\mathbf{4}}$ & $\mathbf{R}_{\mathbf{2}}$, & $\mathbf{R}_{\mathbf{8}}$ \\
\hline Alprazolam & $-\mathrm{CH}_{3}$ & $=\mathrm{N}-$ & $-\mathrm{H}$ & $-\mathrm{H}$ & $-\mathrm{Cl}$ \\
\hline Midazolam & $-\mathrm{CH}_{3}$ & $=\mathrm{C}-$ & $-\mathrm{H}$ & $-\mathrm{F}$ & $-\mathrm{Cl}$ \\
\hline Triazolam & $-\mathrm{CH}_{3}$ & $=\mathrm{N}-$ & $-\mathrm{H}$ & $-\mathrm{Cl}$ & $-\mathrm{Cl}$ \\
\hline \multicolumn{1}{|c|}{$\mathbf{1 , 4 - B e n z o d i a z e p i n e s}$} & $\mathbf{R}_{\mathbf{1}}$ & $\mathbf{R}_{\mathbf{2}}$ & $\mathbf{R}_{\mathbf{3}}$ & $\mathbf{R}_{2}$, & $\mathbf{R}_{\mathbf{8}}$ \\
\hline 7-Amino-flunitrazepam (metabolite) & $-\mathrm{CH}_{3}$ & $=\mathrm{O}$ & $-\mathrm{H}$ & $-\mathrm{F}$ & $-\mathrm{NH}_{2}$ \\
\hline Chlordiazepoxide & ---- & $-\mathrm{NHCH}_{3}$ & $-\mathrm{H}$ & $-\mathrm{H}$ & $-\mathrm{Cl}$ \\
\hline Clonazepam & $-\mathrm{H}$ & $=\mathrm{O}$ & $-\mathrm{H}$ & $-\mathrm{Cl}$ & $-\mathrm{NO}_{2}$ \\
\hline Diazepam & $-\mathrm{CH}_{3}$ & $=\mathrm{O}$ & $-\mathrm{H}$ & $-\mathrm{H}$ & $-\mathrm{Cl}$ \\
\hline Flunitrazepam & $-\mathrm{CH}$ & $=\mathrm{O}$ & $-\mathrm{H}$ & $-\mathrm{F}$ & $-\mathrm{NO}$ \\
\hline Lorazepam & $-\mathrm{H}$ & $=\mathrm{O}$ & $-\mathrm{OH}$ & $-\mathrm{Cl}$ & $-\mathrm{Cl}$ \\
\hline Nordiazepam (metabolite) & $-\mathrm{H}$ & $=\mathrm{O}$ & $-\mathrm{H}$ & $-\mathrm{H}$ & $-\mathrm{Cl}$ \\
\hline Oxazepam (metabolite) & $-\mathrm{H}$ & $=\mathrm{O}$ & $-\mathrm{OH}$ & $-\mathrm{H}$ & $-\mathrm{Cl}$ \\
\hline
\end{tabular}

Table 2: R groups for eleven benzodiazepines on base structures

The purpose of this research is to demonstrate proof of concept of SERS as a sensitive analytical technique for the detection and identification of trace quantities of 
benzodiazepines in aqueous solutions, which can then be adapted for trace detection in toxicological samples. Gold colloidal solutions were used in this study because they were found to provide signal enhancement for all of the benzodiazepines examined in this study. The sensitivity of the technique was optimized by examining the parameters for the SERS detection, including the aggregating agent, its counter ion and its concentration.

\subsection{Experimental}

DEA-exempt, Cerrilliant benzodiazepine solutions (alprazolam LOT\# FE11021101, chlordiazepoxide LOT\# FE062310-01, clonazepam LOT\# FE032311-03, diazepam LOT\# FE082310-03, flunitrazepam LOT\# FE080311-01, lorazepam LOT\# FE03251101, midazolam LOT\# FE100110-01, nordiazepam LOT\# FE110410-01, oxazepam LOT\# FE111710-02, triazolam LOT\# FE122110-03) and Lipomed benzodiazepine solution (7amino-flunitrazepam LOT\# 74.1B2.1L2) were obtained from Fisher Scientific. These samples were solutions in methanol with a concentration of $1 \mathrm{mg} / \mathrm{mL}$. Standards were dried down under nitrogen and then rediluted to $250 \mu \mathrm{g} / \mathrm{mL}$ in $10 \%$ methanol. These stock solutions were then used to prepare stock standards in $10 \%$ methanol ranging in concentration from $50 \mathrm{ng} / \mathrm{mL}$ to $100 \mu \mathrm{g} / \mathrm{mL}$.

Gold colloids were prepared following the modified method of Lee and Meisel proposed by Cyrankieqicz ${ }^{39,36}$ using the reduction of hydrogen tetrachloroaurate (Acros Organics LOT\# A0315355) with a 1\% sodium citrate (EM Science LOT\# 126409120694) solution. Nanoparticles were characterized by measuring ultraviolet-visible absorbance (Varian Cary 100 Bio UV-visible spectrophotometer) as well as physically measuring particle size using scanning electron microscopy (JEOL JSM 5900LV, $3.0 \mathrm{~nm}$ resolution). The following solutions of different aggregating agents were prepared at the 
following concentrations: $3.34 \mathrm{M}, 1.67 \mathrm{M}$, and $0.84 \mathrm{M}$ of $\mathrm{MgCl}_{2}$, and $1.67 \mathrm{M}$ of $\mathrm{CaCl}_{2}$, $\mathrm{KCl}$, and $\mathrm{NaCl}$. All solutions were prepared with ultrapure water. Glassware was cleaned with detergent followed by deionized water and then methanol.

Urine was spiked with 7-amino-flunitrazepam, diazepam, and nordiazepam at concentrations ranging from of $25 \mathrm{ng} / \mathrm{mL}$ to $500 \mathrm{ng} / \mathrm{mL}$ (IRB-13-0035). Liquid-liquid extraction was performed as follows: $1 \mathrm{~mL}$ spiked urine was mixed with $100 \mu \mathrm{L}$ of 100 $\mathrm{mM}$ buffered phosphate at $\mathrm{pH}$ of 6 . This was extracted in $0.5 \mathrm{~mL}$ of ethyl acetate. 100 $\mu \mathrm{L}$ of $1 \mathrm{M} \mathrm{NaOH}$ was then added to the aqueous phase and extracted again with $0.5 \mathrm{~mL}$

of ethyl acetate. The organic phases were dried down and reconstituted in $100 \mu \mathrm{L}$ of $10 \%$ methanol.

For SERS experiments $980 \mu \mathrm{L}$ of gold colloids were placed in sample vial followed by $10 \mu \mathrm{L}$ of the aggregating agent. This was vortexed for 30 seconds. $10 \mu \mathrm{L}$ of drug sample solution was then added and vortexed for 30 seconds. ${ }^{36}$

SERS spectra were obtained with a Perkin Elmer Raman Station 400F with 785 $\mathrm{nm}$ excitation for four spectral scans each at a 10 second exposure. The power at the sample surface was $100 \mathrm{~mW}$. Samples were transferred to SERS inactive quartz cuvettes for analysis.

\subsection{Results and Discussion}

\subsubsection{Nanoparticle Characterization}

The gold nanoparticles produced using the modified Lee Meisel method were dark red in color with an absorbance maximum of $522 \mathrm{~nm}$ (Fig. 23). This is consistent with a particle size of approximately $30 \mathrm{~nm}$. This particle size was confirmed further through the use of a scanning electron microscope. 


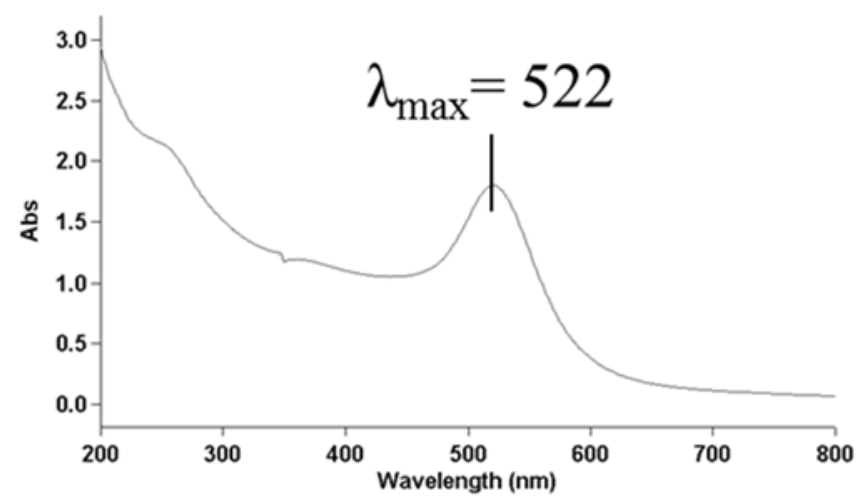

Figure 23: UV-Visible spectra of gold nanoparticles, $30 \mathrm{~nm}$ particles

\subsubsection{Comparison of Different Aggregating Agents}

Four aggregating agents, $\mathrm{MgCl}_{2}, \mathrm{CaCl}_{2}, \mathrm{KCl}$, and $\mathrm{NaCl}$ were compared to optimize the detection of all eleven benzodiazepines. The optimum aggregating agent should provide detection of all compounds at the lowest possible concentration as well as producing a linear response. Furthermore since our goal was to achieve ultra-trace detection for application in drug facilitated sexual assault, we targeted a limit of detection of $50 \mathrm{ng} / \mathrm{mL}$ or lower. An initial stock concentration of $1.67 \mathrm{M}$ was used for all four agents and diluted as necessary. The initial color of the gold nanoparticles was a dark red and following aggregation the solution color varied from dark purple to a colorless solution with gray particles that were visible to the naked eye.

Fig. 24 shows the SERS spectra of all 11 benzodiazepines from 1800 to $400 \mathrm{~cm}^{-1}$ using $\mathrm{MgCl}_{2}$ aggregation at $1.67 \mathrm{M}$. It was found that for all drugs tested, the resulting SERS spectra was did not change with aggregating agent. These results indicate that all four of the aggregate solutions were producing similar interactions at the interface between the nanoparticle and drug. This was important because in order to develop consistent calibration curves, a single high intensity peak was used for each drug. 
Throughout these experiments, all eleven compounds produced unique SERS spectra, facilitating the visual identification of each compound. As noted in Fig. 22 and Table 2, the structures of these compounds are very similar, in some cases having a difference of a single functional group. For example, the only structural difference between flunitrazepam and 7-amino-flunitrazepam is that the amino group on the $\mathrm{R}_{8}$ location of flunitrazepam is reduced to an amine group. This produces the different SERS spectra illustrated in Fig. 24. The ability to identify these compounds by their spectral differences can be of great value in an initial drug screen.

Calibration curves were created for each benzodiazepine with each aggregating agent using the spectral peaks indicated in Fig. 24. Linear dynamic ranges were reported and limits of detection were calculated from calibration curves for all eleven benzodiazepines (Table 3). The sensitivity enhancement produced by the different aggregates varied with each specific drug. 


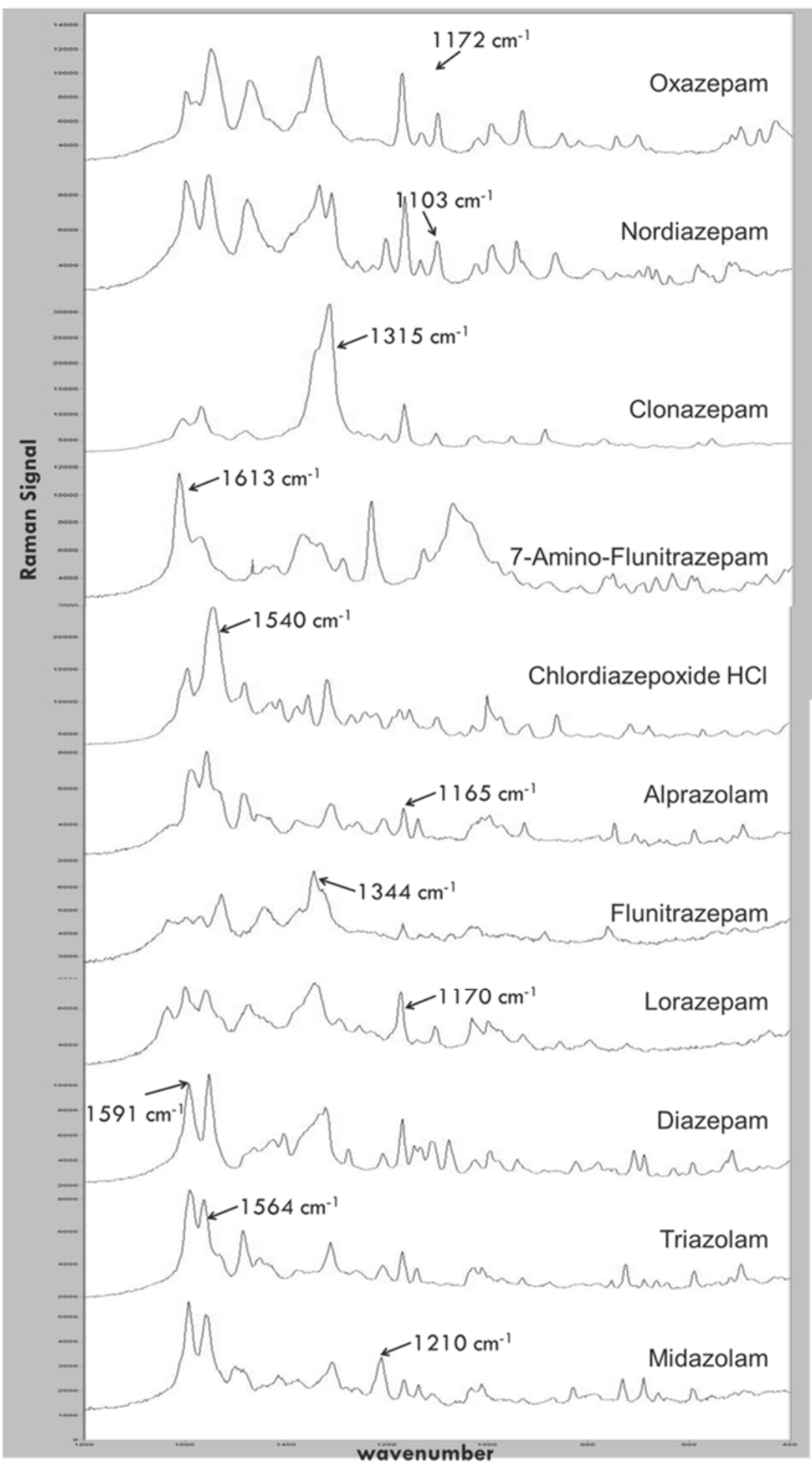

Figure 24: SERS spectra at $250 \mathrm{ng} / \mathrm{mL}$ with $\mathrm{MgCl}_{2}$ (1.67M) aggregation; indicated peaks were used for calibration curves 


\begin{tabular}{|c|c|c|c|c|c|c|}
\hline Drug & Aggregate & $\begin{array}{c}\text { Dynamic } \\
\text { Range } \\
\text { (ng/mL) }\end{array}$ & R-squared & $\begin{array}{l}\text { Standard } \\
\text { Deviation } \\
\text { of Blank } \\
\end{array}$ & Slope & \begin{tabular}{|c|} 
LOD \\
3xSD/Slope \\
ng/mL \\
\end{tabular} \\
\hline \multirow{4}{*}{ Alprazolam } & $\mathrm{MgCl}_{2}$ & $2.5-50$ & 0.9843 & 33 & 29.9 & 3.3 \\
\hline & $\mathrm{CaCl}_{2}$ & $25-100$ & 0.9869 & 63 & 12.5 & 15 \\
\hline & $\mathrm{KCl}$ & $50-500$ & 0.9450 & 140 & 4.40 & 97 \\
\hline & $\mathrm{NaCl}$ & $50-500$ & 0.9628 & 57 & 2.17 & 79 \\
\hline \multirow{4}{*}{$\begin{array}{l}\text { 7-Amino- } \\
\text { Flunitrazepam }\end{array}$} & $\mathrm{MgCl}_{2}$ & $1-25$ & 0.8143 & 260 & 1710 & 0.5 \\
\hline & $\mathrm{CaCl}_{2}$ & $1-25$ & 0.9772 & 1700 & 617 & 8.1 \\
\hline & $\mathrm{KCl}$ & $50-250$ & 0.9580 & 88 & 98.0 & 2.7 \\
\hline & $\mathrm{NaCl}$ & $25-500$ & 0.8704 & 1000 & 15.3 & 200 \\
\hline \multirow{2}{*}{$\begin{array}{c}\text { Chlordiazepoxide } \\
\text { HCl }\end{array}$} & $\mathrm{MgCl}_{2}$ & $100-1000$ & 0.9860 & 790 & 18.5 & 130 \\
\hline & $\mathrm{NaCl}$ & $50-1000$ & 0.8796 & 420 & 2.15 & 590 \\
\hline \multirow{2}{*}{ Clonazepam } & $\mathrm{MgCl}_{2}$ & $100-500$ & 0.8252 & 280 & 18.9 & 44 \\
\hline & $\mathrm{KCl}$ & $100-1000$ & 0.7412 & 140 & 19.3 & 22 \\
\hline \multirow{4}{*}{ Diazepam } & $\mathrm{MgCl}_{2}$ & $2.5-25$ & 0.9936 & 130 & 205 & 1.9 \\
\hline & $\mathrm{CaCl}_{2}$ & $2.5-25$ & 0.9023 & 230 & 182 & 3.7 \\
\hline & $\mathrm{KCl}$ & $50-500$ & 0.8742 & 122 & 17.6 & 21 \\
\hline & $\mathrm{NaCl}$ & $25-1000$ & 0.9360 & 370 & 5.71 & 190 \\
\hline \multirow{3}{*}{ Flunitrazepam } & $\mathrm{MgCl}_{2}$ & $250-2500$ & 0.9138 & 310 & 7.70 & 120 \\
\hline & $\mathrm{CaCl}_{2}$ & $250-2500$ & 0.9191 & 33 & 6.51 & 15 \\
\hline & $\mathrm{NaCl}$ & $100-2500$ & 0.8043 & 100 & 0.572 & 520 \\
\hline \multirow{3}{*}{ Lorazepam } & $\mathrm{MgCl}_{2}$ & $250-1000$ & 0.9429 & 93 & 5.72 & 49 \\
\hline & $\mathrm{CaCl}_{2}$ & $50-1000$ & 0.9872 & 9.0 & 2.75 & 9.8 \\
\hline & $\mathrm{KCl}$ & $250-1000$ & 0.9794 & 100 & 7.59 & 40 \\
\hline \multirow{3}{*}{ Oxazepam } & $\mathrm{MgCl}_{2}$ & $25-500$ & 0.9702 & 120 & 8.10 & 45 \\
\hline & $\mathrm{CaCl}_{2}$ & $100-1000$ & 0.9765 & 220 & 6.43 & 100 \\
\hline & $\mathrm{NaCl}$ & $50-1000$ & 0.9916 & 310 & 2.01 & 470 \\
\hline \multirow{3}{*}{ Nordiazepam } & $\mathrm{MgCl}_{2}$ & $5-50$ & 0.9972 & 110 & 97.1 & 3.3 \\
\hline & $\mathrm{CaCl}_{2}$ & $25-100$ & 0.9216 & 480 & 9.62 & 150 \\
\hline & $\mathrm{NaCl}$ & $50-500$ & 0.8496 & 230 & 1.26 & 540 \\
\hline \multirow{4}{*}{ Triazolam } & $\mathrm{MgCl}_{2}$ & $2.5-50$ & 0.9574 & 73 & 62.6 & 3.5 \\
\hline & $\mathrm{CaCl}_{2}$ & $5-100$ & 0.9239 & 90 & 33.8 & 8.0 \\
\hline & $\mathrm{KCl}$ & $25-100$ & 0.9602 & 400 & 47.3 & 26 \\
\hline & $\mathrm{NaCl}$ & $25-250$ & 0.9413 & 200 & 18.1 & 34 \\
\hline \multirow{3}{*}{ Midazolam } & $\mathrm{MgCl}_{2}$ & $10-100$ & 0.8902 & 18 & 18.7 & 2.9 \\
\hline & $\mathrm{CaCl}_{2}$ & $25-100$ & 0.9271 & 140 & 15.3 & 27 \\
\hline & $\mathrm{KCl}$ & $50-500$ & 0.7633 & 140 & 0.809 & 530 \\
\hline
\end{tabular}

Table 3: Figures of Merit 
$\mathrm{KCl}$ aggregation at $1.67 \mathrm{M}$ provided spectra for only seven of the eleven compounds within the concentration range tested $(1-1000 \mathrm{ng} / \mathrm{mL})$. For these seven drugs the limits of detection ranged from 25 to $250 \mathrm{ng} / \mathrm{mL}$. NaCl provided spectra for eight of the eleven compounds within the concentration range tested with limits of detection ranging from 25 to $100 \mathrm{ng} / \mathrm{mL}$. $\mathrm{CaCl}_{2}$ provided spectra for nine of the eleven drugs tested. The limits of detection for this aggregating agent ranged from 1 to $250 \mathrm{ng} / \mathrm{mL}$. All three of these aggregating agents produced spectra for some of the benzodiazepines tested within $50 \mathrm{ng} / \mathrm{mL}$, however, a more universal aggregation agent was needed to permit detection all the analytes of interest. $\mathrm{MgCl}_{2}$ provided this result. Not only is it shown to produce the lowest limits of detection overall $(0.5-250 \mathrm{ng} / \mathrm{mL})$, it also produced identifying spectra for all eleven analytes of interest within the concentration range tested.

Generalizations can be made about the different effects of these aggregates. The more chlorine ions present, the higher the signal enhancement observed. In general, $\mathrm{MgCl}_{2}$ and $\mathrm{CaCl}_{2}$ provided higher signal intensities for the same drug at the same concentration when compared with $\mathrm{NaCl}$ and $\mathrm{KCl}$. This can be observed in Fig. 25. Shown are calibration curves for alprazolam using $\mathrm{MgCl}_{2}$ and $\mathrm{NaCl}$ as aggregating agents. At $50 \mathrm{ng} / \mathrm{mL}$ of alprazolam, a higher signal intensity is observed for $\mathrm{MgCl}_{2}$ than for $\mathrm{NaCl}$. This has been reported in the literature ${ }^{25}$ to be due to the chloride ion effect. Sodium citrate is used as a reducing agent to form the gold nanoparticles as well as stabilize their formation. Aggregation results when the chlorine ions displace the citrate stabilizer causing particle interactions with the resultant Raman enhancement. This presence of the aggregating agent also facilitates an increase in the signal intensity by 
affecting the ionic strength of the surrounding solution and the surface charge of the substrate. ${ }^{64}$

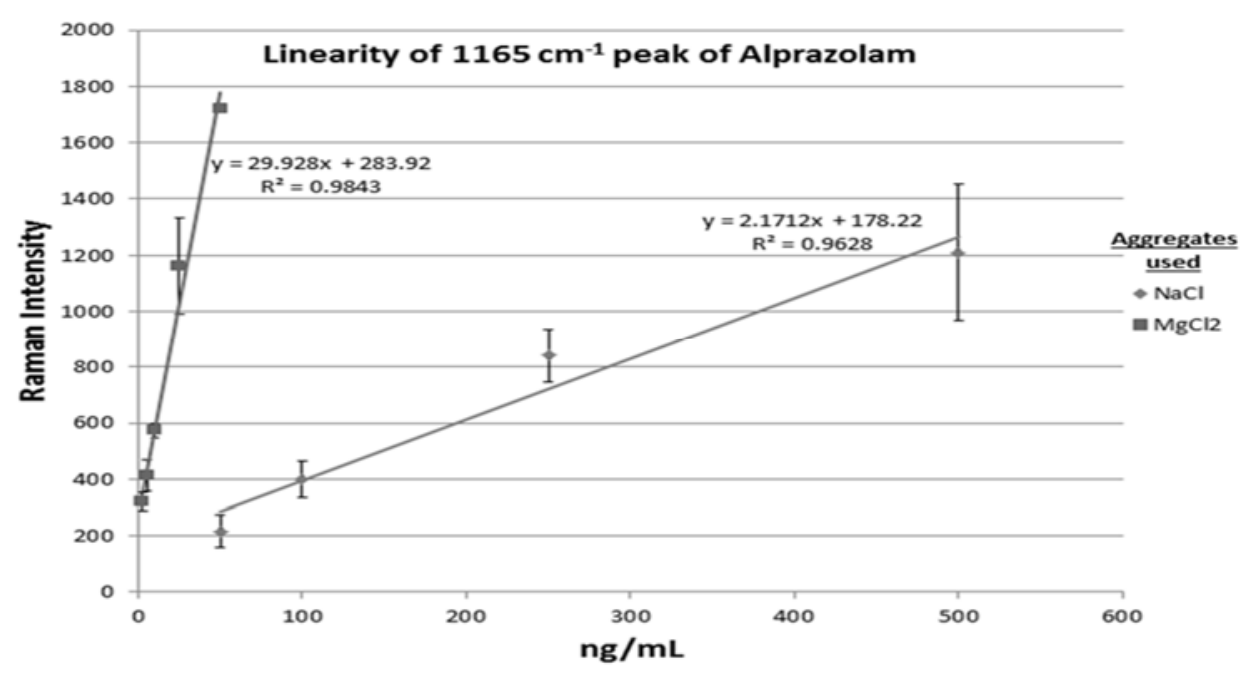

Figure 25: Comparison of $\mathrm{MgCl}_{2}$ and $\mathrm{NaCl}$ aggregation for alprazolam detection

It was also noted that the cation may contribute to the enhancement as well.

$\mathrm{MgCl}_{2}$ and $\mathrm{KCl}$ provided higher intensity signals when compared to $\mathrm{CaCl}_{2}$ and $\mathrm{NaCl}$, respectively. This can be observed in Fig. 26. Shown are calibration curves for alprazolam using $\mathrm{MgCl}_{2}$ and $\mathrm{CaCl}_{2}$ as aggregating agents. At $25 \mathrm{ng} / \mathrm{mL}$ of alprazolam a higher signal intensity is observed for $\mathrm{MgCl}_{2}$ than for $\mathrm{CaCl}_{2}$. This effect has been noted in the literature ${ }^{37}$ and may be due to the size of the cation; however is not as well studied as the effect of anions on the SERS signal. Smaller cations facilitated the nanoparticles to move closer together, increasing the likelihood of hotspot formation, increasing signal intensity. $\mathrm{MgCl}_{2}$ was shown to produce the lowest limits of detection both observable (1 $-250 \mathrm{ng} / \mathrm{mL})$ as well as calculated $(0.5-100 \mathrm{ng} / \mathrm{mL})$ across the range of drugs.

Therefore, $\mathrm{MgCl}_{2}$ was the optimum aggregating agent universally, so it was optimized. 


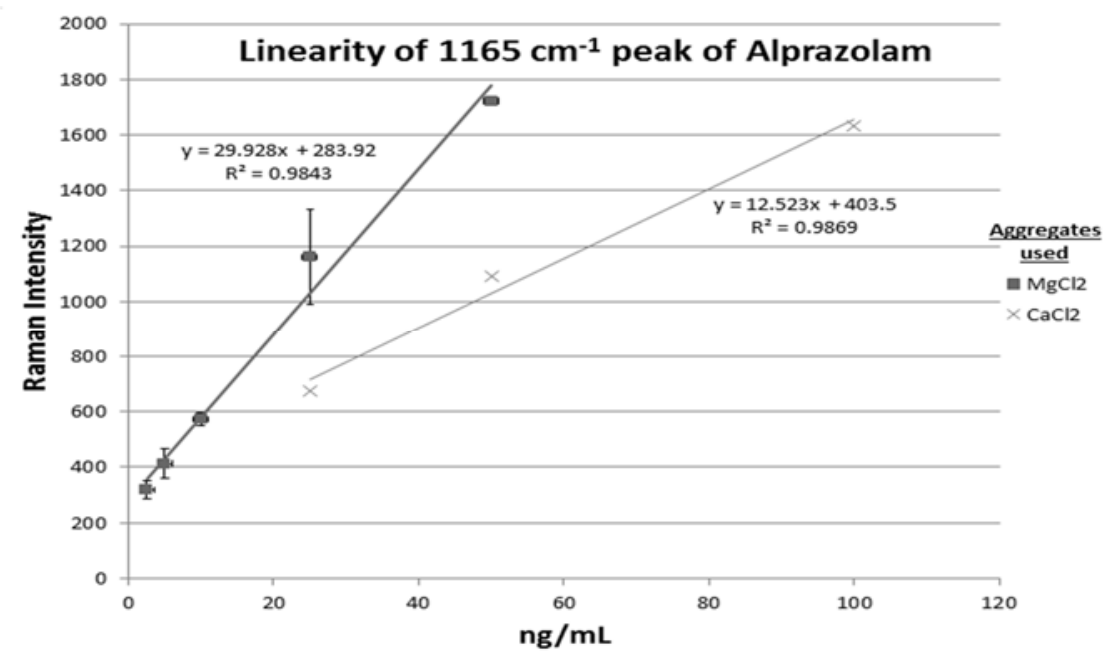

Figure 26: Comparison of $\mathrm{MgCl}_{2}$ and $\mathrm{CaCl}_{2}$ aggregation for alprazolam detection

\subsection{3 $\mathrm{MgCl}_{2}$ Concentration Optimization}

In order to further optimize the detection system, various concentrations (3.34 M, 1.67 $\mathrm{M}$ and $0.84 \mathrm{M}$ ) of $\mathrm{MgCl}_{2}$, were tested to determine if increasing or decreasing the concentration of the agent would affect the SERS signal. For each of the eleven benzodiazepines, the lowest observable concentration obtained in the first part of this study was used. Fig. 27 shows the Raman signal intensity observed for each drug at all three $\mathrm{MgCl}_{2}$ concentrations. 


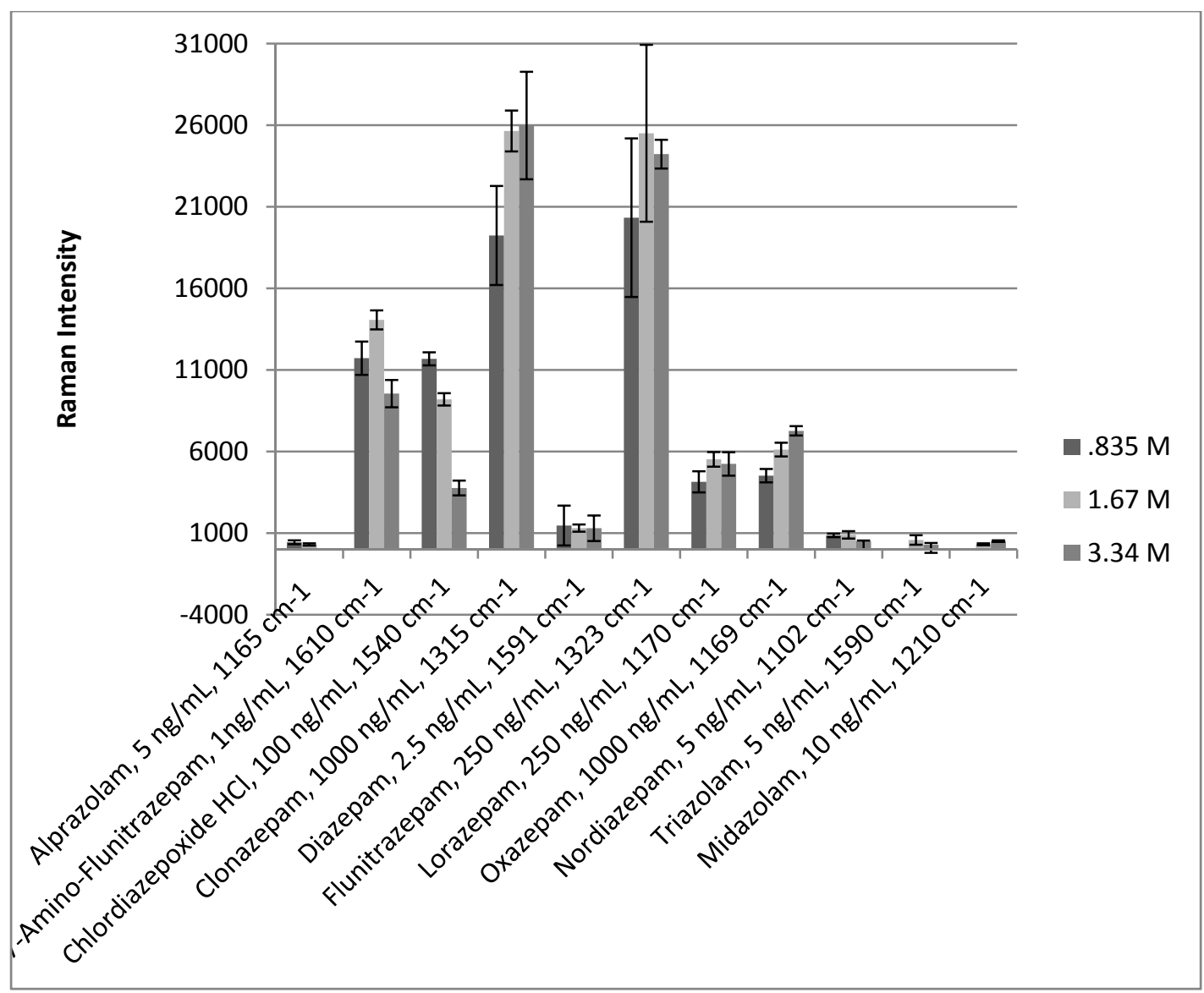

Figure 27: Comparison of 3 concentrations of $\mathrm{MgCl}_{2}$ aggregating reagent for all eleven benzodiazepines at their lowest observable concentration

Although in some cases, the use of $0.84 \mathrm{M} \mathrm{MgCl}_{2}$ (chlordiazepoxide) and $3.34 \mathrm{M}$ $\mathrm{MgCl}_{2}$ (oxazepam) produced a higher intensity signal than $1.67 \mathrm{M} \mathrm{MgCl}_{2}$, certain drugs produced no signal at these lower concentrations. For example the use of $0.84 \mathrm{M} \mathrm{MgCl}_{2}$ produced no signal for triazolam and midazolam and the $3.34 \mathrm{M} \mathrm{MgCl}_{2}$ solution produced no signal for alprazolam. Therefore, $1.67 \mathrm{M} \mathrm{MgCl}_{2}$ was used as the aggregating agent in all further studies. 


\subsubsection{Proof of Concept - Spiked Urine Sample}

Although this technique shows low detection limits in aqueous samples, proof of concept is necessary to show applicability to cases involving real biological samples. In order to do this, a number of the above samples, including 7-amino-flunitrazepam, diazepam, and nordiazepam, were spiked into urine and tested. For example 7-aminoflunitrazepam was spiked into a urine sample at a concentration of $250 \mathrm{ng} / \mathrm{mL}$ and then extracted using the liquid-liquid extraction method described previously in this paper. SERS samples were prepared from the 7-amino-flunitrazepam standard and the urine extract. Fig. 28 shows the SERS spectra produced in both cases. The visual similarity

of the two spectra is clear, and the high intensity peaks at $1233 \mathrm{~cm}^{-1}, 1613 \mathrm{~cm}^{-1}, 1074 \mathrm{~cm}^{-}$ , and $1352 \mathrm{~cm}^{-1}$ peaks can be noted. Three different benzodiazepines, 7-aminoflunitrazepam, diazepam, and nordiazepam, were successfully spiked into urine at concentrations ranging from $25 \mathrm{ng} / \mathrm{mL}$ to $500 \mathrm{ng} / \mathrm{mL}$, extracted by liquid-liquid extraction, and detected using the optimized SERS method. Samples were run in triplicate and calibration curves were produced. All three drugs produced a dynamic range from 25 to $500 \mathrm{ng} / \mathrm{mL}$ with $\mathrm{R}^{2}$ values between 0.98 and 0.92 and limits of detection from $2 \mathrm{ng} / \mathrm{mL}$ to $10 \mathrm{ng} / \mathrm{mL}$, which is below our target of $50 \mathrm{ng} / \mathrm{mL}$. These results demonstrate that benzodiazepines extracted from urine can be detected using this optimized SERS method. 


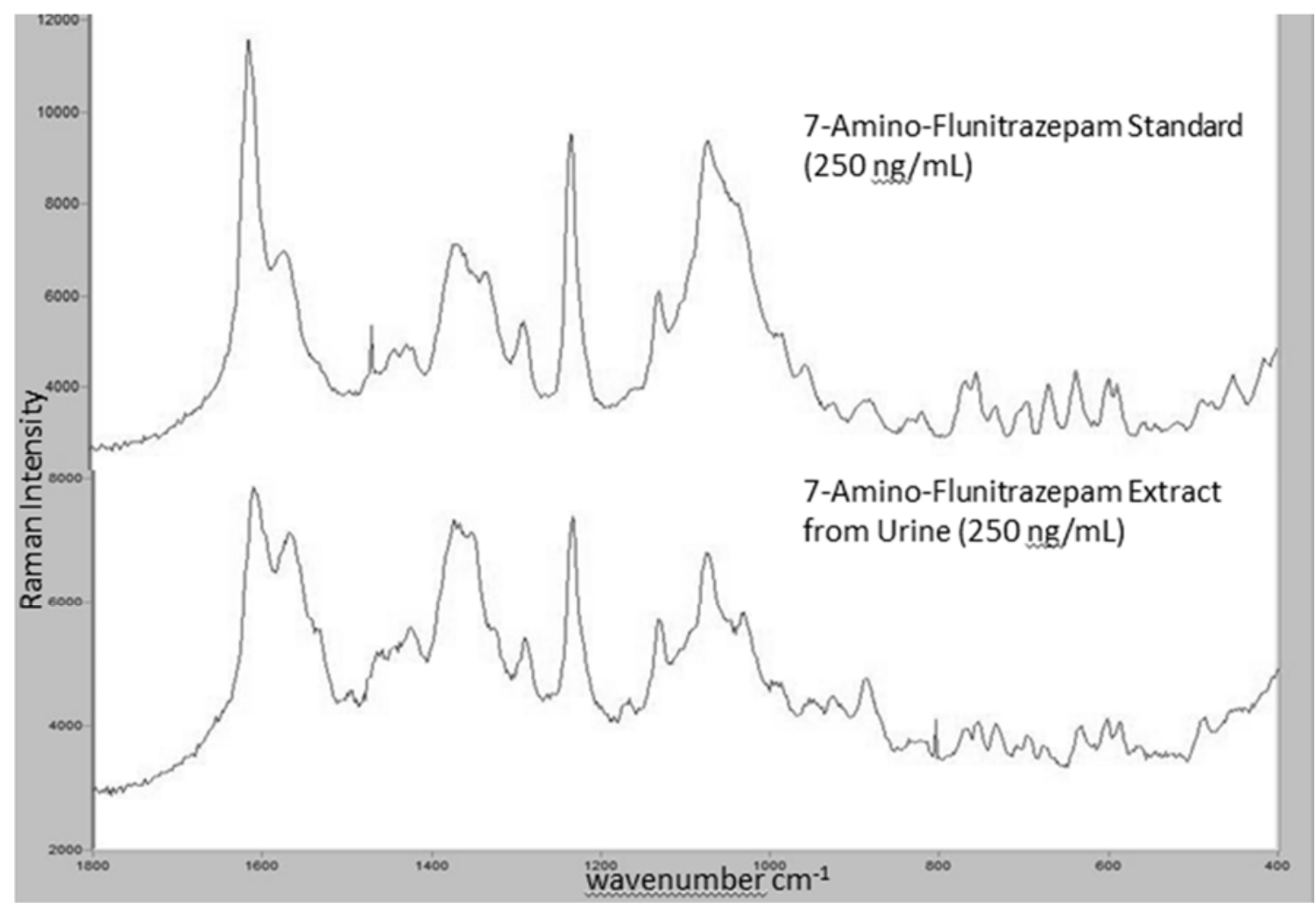

Figure 28: SERS spectra of 7-amino-flunitrazepam standard compared to SERS spectra of 7-amino-flunitrazepam extract from urine

\subsection{Conclusions}

This paper presents an initial assessment of the application of surface enhanced Raman spectroscopy to toxicological samples. SERS spectra were obtained for eleven benzodiazepines of concern in drug facilitated sexual assault. The technique produces enhancement levels several orders of magnitude higher than would be available with standard Raman spectroscopy, permitting trace level analysis of drugs in aqueous solutions. Also, this method overcomes the limitations of some currently available immunoassay techniques by providing lower detection limits and specific spectral based determinations. The method was stable, producing reproducible SERS intensities and frequencies over a wide range of concentrations. 
For all aggregating agents tested, $1.67 \mathrm{M} \mathrm{MgCl}_{2}$ produced the highest signal intensities across all eleven drugs with detection limits ranging from 0.5 to $127 \mathrm{ng} / \mathrm{mL}$ and all. Furthermore, with the exception of chlordiazepoxide and flunitrazepam, detection limits below 50ng/mL were achieved for all tested compounds. This work demonstrates the importance of properly selecting an aggregation agent for SERS detection in suspension. 


\section{SUPPORTED LIQUID EXCTRATION OF BENZODIAZPINES FROM URINE}

\subsection{Introduction}

The detection of illicit drugs in biological matrixes are important in both the clinical and forensic setting. Often, however, the direct analysis of these biological matrixes is not possible due to the complexity of the matrix. Specifically in urine, there are a number of salts, creatinine, urea, proteins, and any number of other exogenous compounds. In order to allow for the detection of an illicit drug of interest, pretreatment and an extraction technique must be employed in order to remove the analyte while leaving behind the rest of the biological matrix. For benzodiazepines, the most common pretreatment method is enzymatic hydrolysis with glucuronidase, a one to two hour process, involving the removal of the drug from the glucuronidated conjugate that is produced in high quantities during the metabolism process. ${ }^{9,10,12,17}$ Other pretreatments involve dilution of the sample with water or a buffer prior to extraction. When extracting benzodiazepines from urine, the two most common methods employed are solid phase extraction (SPE) and liquid-liquid extraction (LLE).

Solid phase extraction uses a solid particle, usually a chromatographic packing material, contained in a cartridge. The ability of the analyte to partition between the liquid phase and the stationary phase causes the retention or elution of that analyte. The packing material chosen depends on the analyte and whether that analyte is retained or eluted from the stationary phase at certain steps along the extraction process. These stationary phases are made up of purified and modified silica. SPE methods normally contains a number of steps: preconditioning the column with an organic, flushing the column to remove the organic, loading a buffered biological sample, washing the loaded 
sample, drying the column, and elution. For some drugs, benzodiazepines in particular, that are highly partitioned from aqueous to organic this extraction can consume more solvent, material, and more time, than a simpler extraction method. ${ }^{5}$ In turn, this costs laboratories more money.

Solid phase extraction has been applied to the extraction of benzodiazepines from a number of biological matrixes. ${ }^{65,66,67,68,69}$ Hegstad et. al. used a mixed mode polymerbases solid phase extraction column to extract benzodiazepines common in Norway form urine samples. This procedure utilized a two hour enzymatic hydrolysis step prior to extraction and involved a four step extraction procedure. The extraction had a recovery range of $51 \%-89 \%$ at an approximate concentration of $150 \mu \mathrm{g} / \mathrm{mL}$ determined using liquid chromatography tandem mass spectroscopy. ${ }^{68}$ This type of SPE method was taken further with the addition of dispersive liquid-liquid microextraction following the SPE extraction. ${ }^{69}$ The dispersive liquid-liquid microextraction utilized the property of benzodiazepines high partitioning from water to chloroform. Post SPE extraction, the eluent was reconstituted in water and chloroform was added and droplets were allowed to form. These droplets now contained the benzodiazepines of interest and could be removed and injected directly into a gas chromatograph for detection. It was found that the addition of the dispersive liquid-liquid microextraction increased the recoveries $(92.5 \%-110 \%)$ compared to methods which just utilized SPE. ${ }^{69}$ While SPE often has good recoveries ( $>50 \%)$ for benzodiazepines, the time and cost involved makes faster simplified extraction techniques viable alternatives for screening for benzodiazepines in urine. 
Liquid-liquid extraction typically involves the isolation of an analyte based on its ability to partition from one phase into another. While liquid-liquid extraction (LLE) is a rapid, easy technique for removing analytes from a biological matrix, many times the analyte of interest is not the only compound which is removed from the biological sample. This poses a problem when using surface-enhanced Raman spectroscopy (SERS) as a detection technique. Interferences, such as other drugs, can be co-extracted and interfere with the analyte spectra. Also, as discussed in Chapter 4, aggregating agents, often salts, and their concentrations can have an effect on the SERS signal. Often salts can be co-extracted into the organic phase of the liquid-liquid extraction, and this can reduce the SERS signal. LLE has been shown to be an efficient method for the isolation of benzodiazepines from both biological and other liquid matrixes. The McCord research group has performed a number of studies which have utilized liquidliquid extraction using a phosphate buffer at a pH of 6 and ethyl acetate to extract benzodiazepines from beverages ${ }^{70,71}$ as well as urine. ${ }^{6,72}$ Since LLE can be an important method for the isolation of interest, a search was made for a method capable of utilizing the benefits of LLE (speed, ease of use, low cost) without many of the downsides (coextractions of interferences and salts).

Supported liquid extraction (SLE) is a cleaner and more efficient extraction method than LLE. It can also provide a sample preparation time of under fifteen minutes. SLE uses a solid silica based diatomaceous earth packed into a column. The use of columns permits this method to become automated. Figure 29 shows the work flow of SLE. The pretreated aqueous biological sample is applied to the top of the column where it spreads over the surface of the support and is absorbed onto a dry column. Unlike solid 
phase extraction, there are no preconditioning steps and no flow-through, reducing sample preparation time and the need for waste containers. ${ }^{73}$ The pretreatment should allowing for the analytes of interest to be present in their neutral form. Once applied, the analytes remain on the surface of the support mimicking the phase interface in LLE.

When the organic extraction solvent is applied, the analytes are then desorbed and the solvent is collected.

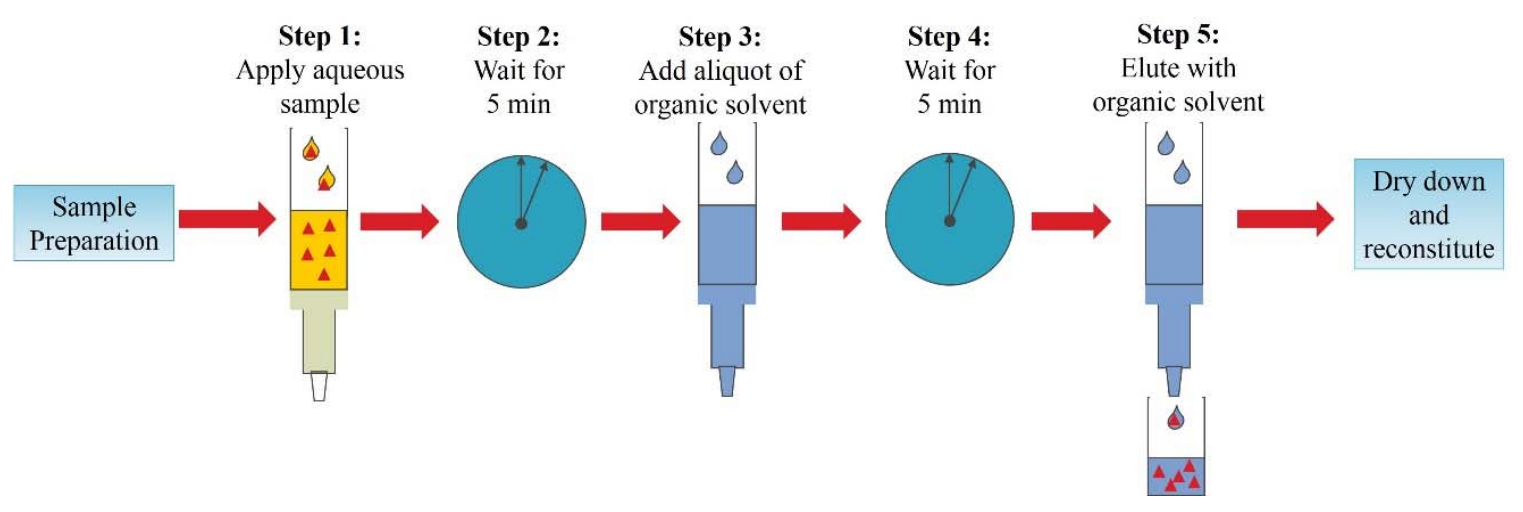

Figure 29: Work flow of supported liquid extraction ${ }^{73}$

Supported liquid extraction has previous been applied to the extraction of benzodiazepines from urine prior to high-performance liquid chromatography ${ }^{74}$ and ultrapressure liquid chromatography followed by tandem mass spectrometry. ${ }^{75}$ Both studies employed a high $\mathrm{pH}$ buffer as a sample preparation step, 9 and 11 respectively. ${ }^{74,75}$ Unfortunately in addition to permitting complete extraction of all benzodiazepines, this procedure will also extract other basic drugs with high pKas. This interference is not a problem when chromatographic methods are used, but when screening for benzodiazepines using (SERS) the number of Raman active interferences needs to be limited. Table 4 lists the eleven benzodiazepines which were tested in this study along with their relevant with $\mathrm{pKa}$ values. The ionization percentages were also calculated 
using an acetate buffer at a $\mathrm{pH}$ of five. After calculations, it was shown that at a $\mathrm{pH}$ of 5.0 all but one of the benzodiazepines of interest in this study, midazolam, have a low percentage of ionization which is ideal for SLE as for optimum extraction of all analytes should be extracted in their neutral state. This low $\mathrm{pH}$ will also allow for the removal of many possible interferences, such a codeine, that have high $\mathrm{pKa}$ values. Percent ionizations were calculated using a simplified version of the Henderson Hasselback equation and a using an acetate buffer at a $\mathrm{pH}$ of five. Equation 9 was used for calculating the percent ionization of the basic $\mathrm{pKa}$, which is the deprotonation of the cationic form of the N-4 on the diazepine ring. Equation 10 was used for calculating the percent ionization of the acidic pKa due to the deprotonation of the $\mathrm{N}-1$ on the diazepine ring to produce an anionic species. ${ }^{26,25}$

$$
\begin{aligned}
\% \text { ionized basic } & =\frac{100}{1+10^{(p H-p K a)}} \\
\% \text { ionized acidic } & =\frac{100}{1+10^{(p K a-p H)}}
\end{aligned}
$$

\begin{tabular}{|c|c|c|c|c|}
\hline Benzodiazepine & $\begin{array}{c}\text { Base } \\
\text { pKa }\end{array}$ & Acid pKa & $\begin{array}{c}\text { \% Ionization } \\
\text { Base }\end{array}$ & $\begin{array}{c}\text { \% Ionization } \\
\text { Acid }\end{array}$ \\
\hline 7-Amino-flunitrazepam & 1.8 & ----- & $0.06 \%$ & ----- \\
\hline Alprazolam & 2.4 & ----- & $0.25 \%$ & ----- \\
\hline Chlordiazepoxide & 4.8 & ----- & $39 \%$ & ----- \\
\hline Clonazepam & 1.5 & 10.5 & $0.03 \%$ & $<0.01 \%$ \\
\hline Diazepam & 3.3 & ----- & $2.0 \%$ & ----- \\
\hline Flunitrazepam & 1.8 & ----- & $0.06 \%$ & ----- \\
\hline Lorazepam & 1.3 & 11.5 & $0.02 \%$ & $<0.01 \%$ \\
\hline Midazolam & 6.2 & ----- & $94 \%$ & ----- \\
\hline Nordiazepam & 3.5 & 12.0 & $3.1 \%$ & $<0.01 \%$ \\
\hline Oxazepam & 1.8 & 11.1 & $0.06 \%$ & $<0.01 \%$ \\
\hline Triazolam & 1.7 & ----- & $0.05 \%$ & ---- \\
\hline
\end{tabular}

Table 4: pKa and ionization data on eleven benzodiazepines 


\subsection{Experimental}

All solutions were prepared with ultrapure water. Glassware was cleaned with detergent followed by deionized water and then methanol. All chemicals not directly listed were purchased from Fisher Scientific. DEA-exempt, Cerrilliant benzodiazepine solutions (alprazolam LOT\# FE110211-01, chlordiazepoxide LOT\# FE062310-01, clonazepam LOT\# FE032311-03, diazepam LOT\# FE082310-03, flunitrazepam LOT\# FE080311-01, lorazepam LOT\# FE032511-01, midazolam LOT\# FE100110-01, nordiazepam LOT\# FE110410-01, oxazepam LOT\# FE111710-02, triazolam LOT\# FE122110-03) and Lipomed benzodiazepine solution (7-amino-flunitrazepam LOT\# 74.1B2.1L2) were obtained from Fisher Scientific. These samples were solutions in methanol with a concentration of $1 \mathrm{mg} / \mathrm{mL}$. Urine was spiked with drug standards at concentrations ranging from of $6 \mathrm{ng} / \mathrm{mL}$ to $25,000 \mathrm{ng} / \mathrm{mL}$ (IRB-13-0035).

Isolute ${ }^{\circledR} \mathrm{SLE}+$ supported liquid extraction $2 \mathrm{~mL}$ and $5 \mathrm{~mL}$ capacity extraction columns were obtained from Biotage. For the $2 \mathrm{~mL}$ extraction columns three different sample preparations methods were used on $1 \mathrm{~mL}$ spiked urine sample. Dilution 1:1 with deionized water $(\mathrm{pH}=6.5)$, dilution $1: 1$ with $100 \mathrm{mM}$ ammonium acetate $\left(\mathrm{NH}_{4} \mathrm{OAc}\right)$ buffer adjusted to $\mathrm{pH} 5.0$ with $1 \%$ formic acid, and dilution with $950 \mu \mathrm{L}$ of $100 \mathrm{mM}$ $\mathrm{NH}_{4} \mathrm{OAc}$ buffer adjusted to $\mathrm{pH} 5.0$ and $50 \mu \mathrm{L} \beta$-glucuronidase at $4500 \mathrm{U} / \mathrm{mL}$ with hydrolysis in water bath at $60^{\circ} \mathrm{C}$ for two hours. ${ }^{73}$ For the $5 \mathrm{~mL}$ extraction columns $2.5 \mathrm{~mL}$ of urine spiked with alprazolam was diluted 1:1 with $100 \mathrm{mM} \mathrm{NH} 4 \mathrm{OAc}$.

The supported liquid extraction was performed as follows for the $2 \mathrm{~mL}$ extraction columns: loaded $2 \mathrm{~mL}$ of pretreated sample onto column with pulsed vacuum to initiate flow, leave for 5 minutes. Two and a half $\mathrm{mL}$ of dichloromethane was applied and 
allowed to flow under gravity for 5 minutes, this was repeated a second time and vacuum was then applied to elute. Samples were then dried under nitrogen and reconstituted in $100 \mu \mathrm{L}$ of $10 \%$ methanol. Each extraction was performed in triplicate. The procedure was similar for the $5 \mathrm{~mL}$ columns however samples were loaded with $5 \mathrm{~mL}$ of pretreated sample and aliquots of extraction solvent were increased to a volume of $5 \mathrm{~mL}$ dichloromethane.

The optimized SERS method and sample preparation using gold colloids and 1.67 $\mathrm{M} \mathrm{MgCl}_{2}$ previously discussed was used to detect the drugs following extraction for this study. ${ }^{6}$ SERS spectra were obtained with a Perkin Elmer Raman Station 400F with 785 $\mathrm{nm}$ excitation for four spectral scans each at a 10 second exposure. The power at the sample surface was $100 \mathrm{~mW}$. Samples were transferred to SERS inactive quartz cuvettes for analysis.

\subsection{Results and Discussion}

\subsubsection{Comparison of sample preparation}

Three different sample preparation methods were tested in order to determine which would extract all eleven benzodiazepines in this study. As discussed in the methods these were dilution of urine sample with water, dilution of urine sample with acetate buffer at a $\mathrm{pH}$ of 5.0, and dilution of urine sample with acetate buffer at a $\mathrm{pH}$ of 5.0 followed by enzyme hydrolysis. Dilution with water was chosen due to the simplicity of the method. The acetate buffer sample preparation was chosen in order to have more control of the $\mathrm{pH}$ (5.0) and ionization of the benzodiazepines in the sample. Finally, enzyme hydrolysis was also tested in order to determine if extraction could be improved by removing the glucuronide conjugates, allowing for more free-drug to be detected. For 
each sample preparation technique, $1 \mathrm{~mL}$ of urine spiked with maximum concentrations of each benzodiazepine $(500-25,000 \mathrm{ng} / \mathrm{mL})$ was used. Figure 30 shows a comparison of the Raman intensities produced for one of the benzodiazepines, 7-aminoflunitrazepam, at $250 \mathrm{ng} / \mathrm{mL}$ in urine comparing the three SLE sample preparation techniques as well as compared to signal produced from liquid-liquid extraction previously published. ${ }^{6}$

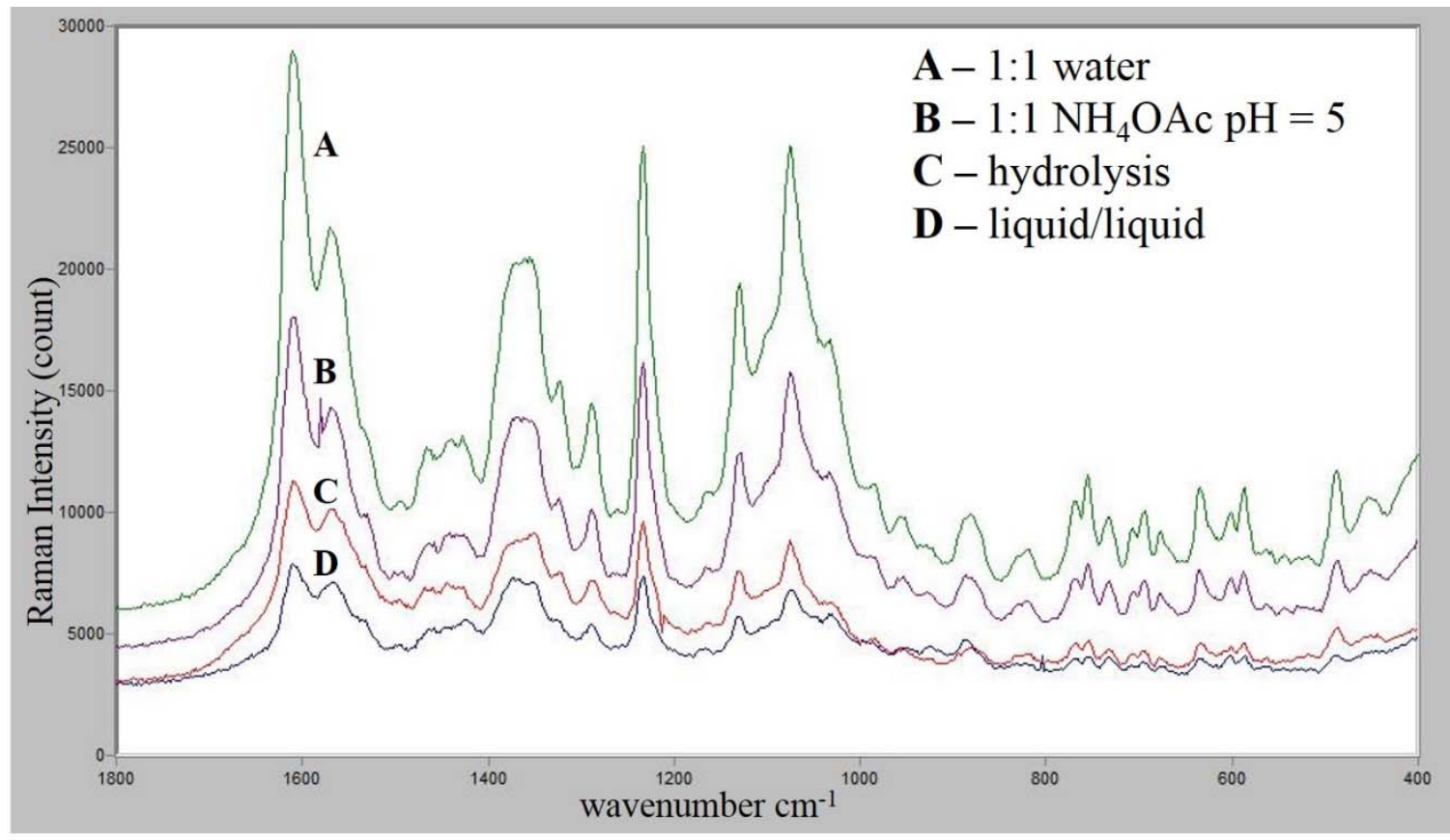

Figure 30: SERS signals for $250 \mathrm{ng} / \mathrm{mL} 7$-amino-flunitrazepam extracted with three SLE preparation techniques and liquid-liquid extraction; All three SLE extractions (A,B,C) produced higher Raman intensities (increased extraction) when compared to the liquidliquid extraction (D) performed in Chapter 4. Dilution with water was the most efficient extraction in the case of this drug while enzyme hydrolysis was the least efficient.

All the SLE preparation techniques provided higher signal intensities than with the previously published liquid-liquid extraction method. It was found that when the 
spiked urine was diluted 1:1 with deionized water, while having the highest signal intensities for some of the benzodiazepines which were efficiently extracted, only 8 of the eleven benzodiazepines extracted. Also four of those that did extract only had very low intensity spectral peaks at maximum concentrations $(500-25,000 \mathrm{ng} / \mathrm{mL})$ which are well above the required $50 \mathrm{ng} / \mathrm{mL}$ limit.

Hydrolysis by $\beta$-glucuronidase provided the lowest signal intensities of the three preparation techniques. Hydrolysis provided similar results to dilution with deionized water in the number of benzodiazepines which efficiently extracted, eight. The three benzodiazepines which were not detectable by SERS post-extraction were alprazolam, midazolam, and triazolam. Another issue with hydrolysis deals with the potential conversion of flunitrazepam to 7-amino-flunitrazepam. In Figure 31, the SERS spectra following hydrolysis and extraction for these two compounds is shown as well as a comparison to the SERS spectra of a pure flunitrazepam sample. The effect of conversion is preventing the identification of flunitrazepam as the drug present. Finally, this procedure adds an additional hour to analysis time. The goal of this project is to reduce the total analysis time necessary for the screening of these types of drugs. 


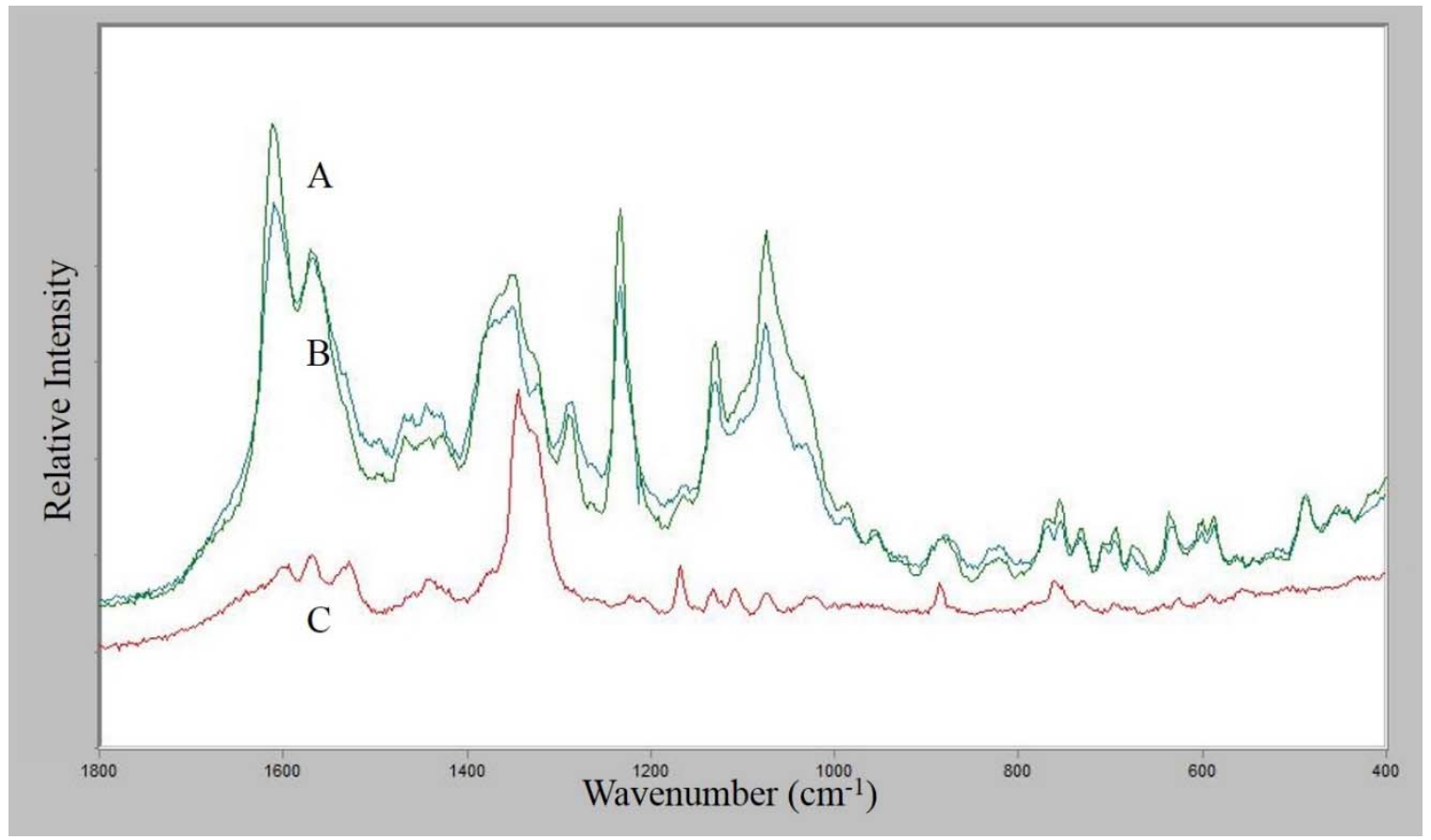

Figure 31: SERS spectra of (A) flunitrazepam and (B) 7-amino-flunitrazepam using hydrolysis sample preparation; compared to SERS spectra of pure (C) flunitrazepam Dilution with ammonium acetate buffer at a $\mathrm{pH}=5.0$ allowed for extraction of all the benzodiazepines in this study. This preparation technique also provided signal intensities greater than those of standard liquid-liquid extraction and the hydrolysis preparation. In addition to a $\mathrm{pH}$ of 5.0 , the $\mathrm{pH}$ of the buffer was also adjusted to 4 and 6 and extraction performed. Neither other $\mathrm{pH}$ improved the signal observed for the benzodiazepines, therefore they did not improve the extraction, thus a $\mathrm{pH}$ of 5.0 was determined to be optimum. Dilution with the ammonium acetate buffer at a $\mathrm{pH}$ of 5.0 was chosen as the optimum preparation for SLE and was used to produce calibration curves and determine limits of detection for this technique. 


\subsubsection{Figures of Merit}

Calibration curves were created for each benzodiazepine following extraction of 1 $\mathrm{mL}$ of spiked urine using the spectral peaks indicated in Table 5. Linear dynamic ranges were reported and limits of detection were calculated from calibration curves for all eleven benzodiazepines (Table 2). This limits of detection were compared to those of the pure drug standards determined in Chapter $4 .^{6}$

\begin{tabular}{|c|c|c|c|c|c|c|}
\hline & \multicolumn{5}{|c|}{ Post Extraction Figures of Merit } & Pure Drug \\
\hline Benzodiazepine & $\begin{array}{c}\text { Dynamic } \\
\text { Range } \\
(\mathrm{ng} / \mathrm{mL})\end{array}$ & $\begin{array}{c}\text { R- } \\
\text { squared }\end{array}$ & $\begin{array}{c}\text { Standard } \\
\text { Deviation } \\
\text { of blank }\end{array}$ & Slope & $\begin{array}{c}\text { LOD } \\
\text { SXD/Slope } \\
(\mathrm{ng} / \mathrm{mL})\end{array}$ & $\begin{array}{c}\text { LOD } \\
(\mathrm{ng} / \mathrm{mL})\end{array}$ \\
\hline $\begin{array}{c}\text { 7-Amino- } \\
\text { flunitrazepam }\end{array}$ & $6-250$ & 0.9342 & 131 & 44.63 & 8.8 & 0.5 \\
\hline Alprazolam & $240-25000$ & 0.9917 & 66 & 0.1122 & 1100 & 3.3 \\
\hline Chlordiazepoxide & $250-4000$ & 0.9845 & 469 & 1.005 & 1400 & 130 \\
\hline Clonazepam & $128-5000$ & 0.9555 & 59 & 0.2185 & 890 & 44 \\
\hline Diazepam & $32-500$ & 0.9896 & 4 & 2.259 & 5.3 & 1.9 \\
\hline Flunitrazepam & $60-2500$ & 0.9920 & 21 & 1.436 & 40 & 120 \\
\hline Lorazepam & $640-10000$ & 0.9791 & 29 & 0.0509 & 1500 & 49 \\
\hline Midazolam & $600-25000$ & 0.9570 & 61 & 0.0171 & 11000 & 3.3 \\
\hline Nordiazepam & $80-500$ & 0.8872 & 42 & 0.4748 & 420 & 3.3 \\
\hline Oxazepam & $80-500$ & 0.8312 & 54 & 0.6812 & 240 & 45 \\
\hline Triazolam & $600-25000$ & 0.9648 & 25 & 0.0193 & 7000 & 2.9 \\
\hline
\end{tabular}

Table 5: Figures of Merit; note the higher limits of detection for the extracted drugs when compared to the pure drugs.

Overall, it was found that limits of detection for all the benzodiazepines studied except for the 1,2-triazolo and 1,2-imdazo benzodiazepines, was similar, though still slightly higher that that determined for the pure samples. Due to no extraction system being one hundred percent efficient, the limits of detection post-extraction are expected to be slightly higher than those of the pure drug. For the two classes of drugs that did not 
produce similar limits of detection between the extracted and pure drug, the 1,2-triazolo and 1,2-imdazo benzodiazepines, the pure drugs had limits of detection between 2.9 $\mathrm{ng} / \mathrm{mL}$ and $3.3 \mathrm{ng} / \mathrm{mL}$, the extracted drug had limits of detection at 240 and $600 \mathrm{ng} / \mathrm{mL}$ respectively. This concentration is ten times the required maximum limit of detection for drug facilitated sexual assault cases. Components of urine, such as urea and creatinine were examined for their interference with the extraction of these three benzodiazepines and were found to not interfere. It was determined that these three benzodiazepines, alprazolam, midazolam and triazolam, were not extracting efficiently. These three compounds are the only benzodiazepines used in this study that contain an additional 1,3diazole ring or 1,2,4-triazole linked to the 1-2 bond of the 1,4-diazepine ring benzodiazepine base structure. The additional nitrogens, when compared to the other benzodiazepines in this study, could be having an effect on the extraction of these drugs. Another important note is specifically for midazolam, which has a pKa of 6 . Table 4 shows that when the percent ionization is calculated for midazolam under the conditions of the acetate buffer $(\mathrm{pH}=5.0)$ used in these experiments, it is $94 \%$ ionized. Due the extraction technique occurring efficiently with the neutral analyte, this high percent ionization for midazolam greatly reduces the efficiency of the extraction. However, if the $\mathrm{pH}$ of the buffer was to be increased, while the percent of ionization would go down, this would also increase the likelihood of other basic drugs being extracted with the benzodiazepines. A number of experiments were performed in an attempt to improve recovery of these compounds in urine.

The amount of urine extracted in the SLE extraction columns was increased from $1 \mathrm{~mL}$ to $2.5 \mathrm{~mL}$ of urine. In order to do this, the size of the extraction cartridge was 
increased from $2 \mathrm{~mL}$ to $5 \mathrm{~mL}$. Samples of alprazolam were spiked into the urine ranging in concentration from $100-25000 \mathrm{ng} / \mathrm{mL}$ following the extraction procedure in the methods for the $5 \mathrm{~mL}$ SLE cartridges. This concentration range is the same as for the 1 $\mathrm{mL}$ urine samples. Samples were then analyzed using the optimized SERS method previously mentioned. It was found that the detection limits did not improve for alprazolam and the noise in the spectrum was increased.

In order to ascertain if extraction efficiency was an issue with alprazolam in particular, infusion mass spectrometry was performed. A spiked urine sample of alprazolam was prepared at $250 \mathrm{ng} / \mathrm{mL}$ and extracted using the optimized $2 \mathrm{~mL}$ SLE cartridge method and standards were prepared at a corresponding $100 \%$ extraction concentration. A Waters Quattro Micro API instrument was used with the following set parameters: capillary voltage $(2.89 \mathrm{kV})$, cone voltage $(45 \mathrm{~V})$, extractor voltage $(3 \mathrm{~V})$, source temperature $\left(125^{\circ} \mathrm{C}\right)$, desolvation temperature $\left(400^{\circ} \mathrm{C}\right)$. The sample was injected at a rate of $25 \mu \mathrm{L} / \mathrm{min}$ with a spectral acquisition time of one second. The instrument was flushed between samples with methanol. Using peak area it was found that alprazolam was only $6 \%$ extracted when compared to the amount extracted from a pure sample (no urine). Therefore, a more specific extraction technique needs to be employed for some of the benzodiazepines, specifically the 1,2-triazolo and 1,2-imdazo benzodiazepines.

\subsection{Conclusions}

Supported liquid extraction coupled with surface enhanced Raman spectroscopy has been shown to provide a rapid (less than twenty minutes) and less complicated method of screening for benzodiazepines in urine at concentrations relevant to drug facilitated sexual assault cases. The detection limits range from $6-640 \mathrm{ng} / \mathrm{mL}$ for the 
benzodiazepines in this study. Other extraction techniques may need to be examined in order to further improve the detection limits for some benzodiazepines, specifically the 1,2-triazolo and 1,2-imdazo benzodiazepines. This technique also has the added benefit of providing spectral information about the compound detected giving investigators information about the specific compound rather than just the class of drugs. 


\section{INTERFERENCE STUDY OF COMMOMLY ENCOUNTERED DRUGS FOUND IN DRUG FACILITATED SEXUAL ASSAULT SAMPLES}

\subsection{Introduction}

While the overall goal of these studies is to optimize the extraction of benzodiazepines from simulated drug facilitated sexual assault sample, consideration needs to be given to other drugs that are commonly present in a urine sample for these cases. Many times victims of such crimes may not have ingested just the criminally administered drug but other compounds such as over the counter medication, acetaminophen, a cup of coffee, caffeine, or prescription medications, such as codeine. ${ }^{10-}$

${ }^{11}$ It is important to determine whether these other common compounds found in toxicological urine samples interfere with either the optimized surface enhanced Raman technique or with the optimized extraction method.

A range of drugs was examined from common over the counter medication, such as acetaminophen and salicylic acid, to illegal drugs such as gammahydroxy buteric acid. Table 6 lists the interference compounds to be examined, the concentration ranges commonly encountered in urine as well each the compound's pKa value. These pKa values range from low (2.97 for salicylic acid) to very high (14 for caffeine). Those compounds with low $\mathrm{pKa}$ were expected to co-extract with the optimized supported liquid extraction (SLE) at a $\mathrm{pH}$ of 5.0. It was also important to examine whether these compounds will produce a SERS spectrum using the optimized method. ${ }^{6}$ For those compounds with high pKas, it was expected that at low $\mathrm{pH}$ these compounds will be retained by the SLE substrate due to being more ionized at the extraction preparation $\mathrm{pH}$ of 5 . 


\begin{tabular}{|c|c|c|}
\hline Drug & Urine Concentration Range & pKa \\
\hline Caffeine & $1-15 \mu \mathrm{g} / \mathrm{mL}^{76}$ & 14 \\
\hline Gammahydroxy buteric acid (GHB) & $1-15 \mu \mathrm{g} / \mathrm{mL}^{77}$ & 4.7 \\
\hline Codeine & $2-62 \mu \mathrm{g} / \mathrm{mL}^{78}$ & 8.2 \\
\hline Nicotine & $0.1-3 \mu \mathrm{g} / \mathrm{mL}^{79}$ & 8.5 \\
\hline Cotinine & $0.1-3 \mu \mathrm{g} / \mathrm{mL}^{79}$ & 8.8 \\
\hline Cocaine & $0.05-10 \mu \mathrm{g} / \mathrm{mL}^{80}$ & 8.6 \\
\hline$\Delta^{9}$-tetrahydrocannabinol & $2.5-240 \mathrm{ng} / \mathrm{mL}^{81}$ & 10.6 \\
\hline Salicylic acid & $0.05-3 \mathrm{mg} / \mathrm{mL}^{82}$ & 2.97 \\
\hline Acetaminophen & $0.005-50 \mu \mathrm{g} / \mathrm{mL}^{83}$ & 9.5 \\
\hline
\end{tabular}

Table 6: Interference compounds studied with common urine concentration and $\mathrm{pKas}$

\subsection{Experimental}

All solutions were prepared with ultrapure water. Glassware was cleaned with detergent followed by deionized water and then methanol. All chemicals not directly listed were purchased from Fisher Scientific. DEA-exempt, Cerrilliant drug solutions (codeine LOT\# FE052011-04, cotinine LOT\# FN061710-01, cocaine HCl LOT\# FE051012-01, gammahydroxy buteric acid (GHB) LOT\# b018, and $\Delta^{9}-$ tetrahydrocannabinol (THC) LOT\# FE121112-02), Lipomed benzodiazepine solution (7amino-flunitrazepam LOT\# 74.1B2.1L2), and Crescent Chemical Co. Inc drug solution (nicotine LOT\# 04CC115) were obtained from Fisher Scientific. These samples were solutions in methanol with a concentration of $1 \mathrm{mg} / \mathrm{mL}$. Acetaminophen (Acros LOT \# A0317675), salicylic acid, and caffeine were powdered compounds obtained from Fisher Scientific.

Drug standard dilutions were prepared in concentration ranges as follows in $10 \%$ methanol: GHB $(10 \mu \mathrm{g} / \mathrm{mL}-150 \mu \mathrm{g} / \mathrm{mL})$, caffeine $(15 \mu \mathrm{g} / \mathrm{mL}-75 \mu \mathrm{g} / \mathrm{mL})$, codeine $(10$ $\mu \mathrm{g} / \mathrm{mL}-310 \mu \mathrm{g} / \mathrm{mL})$, cotinine and nicotine $(0.5 \mu \mathrm{g} / \mathrm{mL}-15 \mu \mathrm{g} / \mathrm{mL})$, salicylic acid $(0.05$ $\mathrm{mg} / \mathrm{mL}-1.5 \mathrm{mg} / \mathrm{mL})$, acetaminophen $(0.025 \mu \mathrm{g} / \mathrm{mL}-250 \mu \mathrm{g} / \mathrm{mL})$, cocaine $(0.25 \mu \mathrm{g} / \mathrm{mL}$ 
$-50 \mu \mathrm{g} / \mathrm{mL})$, and THC $(10 \mathrm{ng} / \mathrm{mL}-1250 \mathrm{ng} / \mathrm{mL})$. These concentration ranges take into account the increase in concentration following extraction to mimic the results of an extracted sample.

Urine was spiked with interference drug standards at the range of concentrations reported in Table 6 as well as with 7-amino-flunitrazepam standard at a concentration of $250 \mathrm{ng} / \mathrm{mL}$ (IRB-13-0035). Isolute ${ }^{\circledR} \mathrm{SLE}+$ supported liquid extraction $2 \mathrm{~mL}$ capacity extraction columns were obtained from Biotage. $1 \mathrm{~mL}$ of spiked urine sample was diluted 1:1 with $100 \mathrm{mM}$ ammonium acetate $\left(\mathrm{NH}_{4} \mathrm{OAc}\right)$ buffer adjusted to $\mathrm{pH} 5.0$ with $1 \%$ formic acid. ${ }^{73}$ The supported liquid extraction was performed as follows: loaded 2 $\mathrm{mL}$ of pretreated sample onto column with pulsed vacuum to initiate flow, leave for 5 minutes. $2.5 \mathrm{~mL}$ of dichloromethane was applied and allowed to flow under gravity for 5 minutes, this was repeated a second time and vacuum was then applied to elute. Samples were then dried under nitrogen and reconstituted in $100 \mu \mathrm{L}$ of $10 \%$ methanol. Each extraction was performed in triplicate.

The optimized SERS method and sample preparation using gold colloids and 1.67 $\mathrm{M} \mathrm{MgCl}_{2}$ previously discussed was used to detect the pure drugs as well as following extraction for this study. ${ }^{6}$ SERS spectra were obtained with a Perkin Elmer Raman Station $400 \mathrm{~F}$ with $785 \mathrm{~nm}$ excitation for four spectral scans each at a 10 second exposure. The power at the sample surface was $100 \mathrm{~mW}$. Samples were transferred to SERS inactive quartz cuvettes for analysis. 


\subsection{Results and Discussion}

\subsubsection{Pure interference compounds}

A range of concentrations of each interferant was tested to see if SERS spectra would be produced using the established optimized method. ${ }^{6}$ This was done to assess whether the extraction method for the benzodiazepines was needed to remove these compounds and if co-extraction did occur, would these drugs interfere in obtaining the spectra of benzodiazepines. Of the nine most common interferences, caffeine, GHB, cocaine, salicylic acid, and acetaminophen did not produce any SERS signal using the optimized method and over the biologically relevant concentrations. Therefore these five compounds should not cause interference when present in a urine sample. The other four compounds, nicotine, cotinine, codeine and THC did produce strong SERS signals and each will each be discussed in detail.

Nicotine and it's major metabolite cotinine do not produce a spectra containing a great number of peaks but both compounds had a high intensity, sharp peak at $1030 \mathrm{~cm}^{-1}$ (Figure 32). Calibration curves were prepared using the peak at $1030 \mathrm{~cm}^{-1}$ for intensity measurements, over the range of concentrations and it was found that both drugs had portions of the curve above the linear range for the detection of these two drugs. If the linear range was established, limits of detection could be determined and this SERS method could be used for the detection of nicotine and cotinine. This limit of detection would be well below the lowest concentration prepared here $(0.5 \mu \mathrm{g} / \mathrm{mL})$ due to the high signal intensities. Previous work has performed using SERS to detect nicotine which used hydroxylamine reduced silver nanoparticles in a polydimethylsiloxane microfluidic device to gain detection limits of $0.1 \mu \mathrm{g} / \mathrm{mL} .{ }^{84}$ Gold nanoparticles may improve this 
analysis. Due to the pKa of these compounds (8.5 and 8.8), the optimized supported liquid extraction method which occurs at a $\mathrm{pH}=5.0$, should remove these compounds when extracting benzodiazepines.

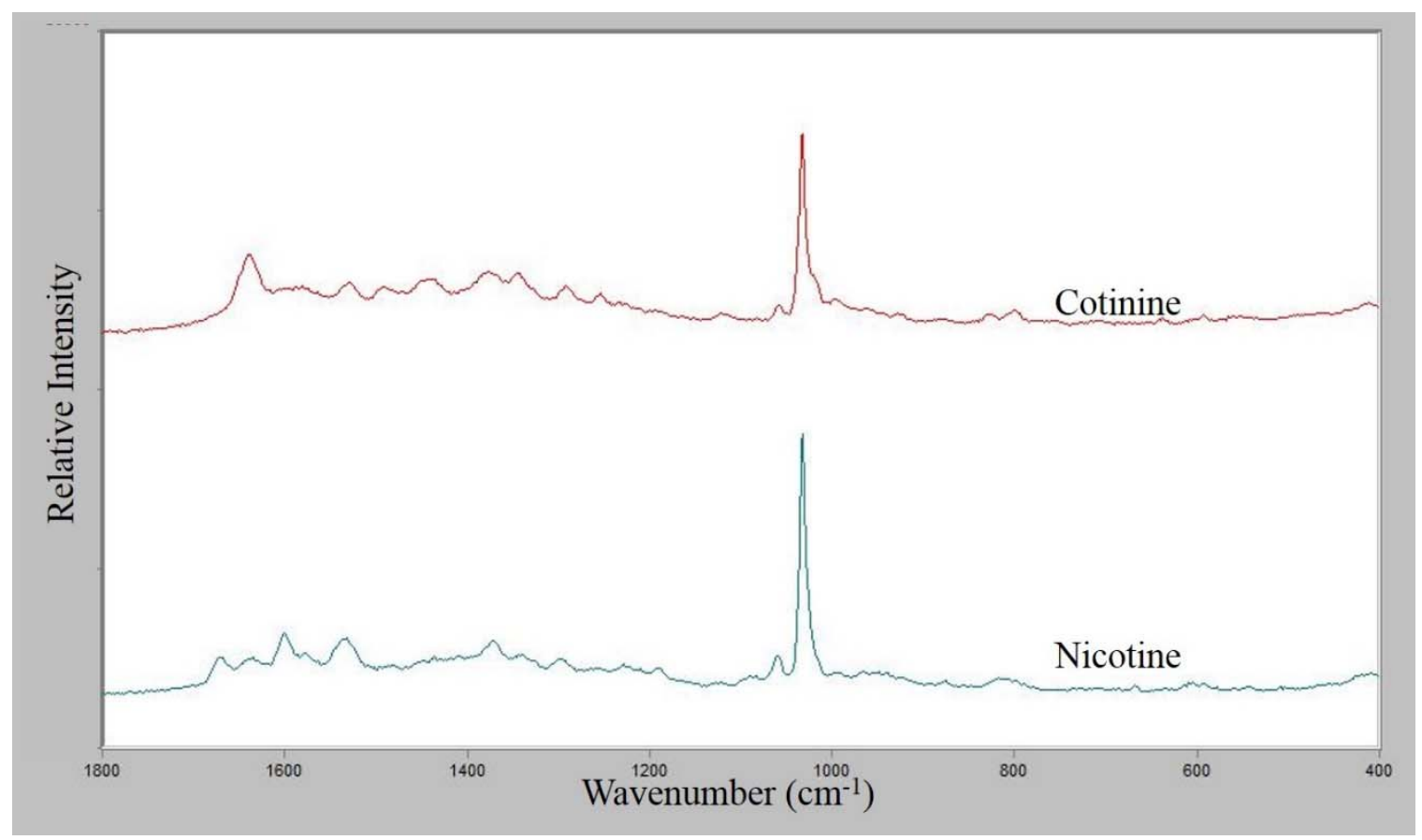

Figure 32: SERS of nicotine and cotinine, $5 \mathrm{ng} / \mathrm{mL}$ each

Codeine produced a very strong spectra rich with spectral peaks (Figure 33). The peak at $1430 \mathrm{~cm}^{-1}$ was used to measure intensity to prepare a calibration curve. It was found that all concentrations of codeine prepared were above the linear range of the compound, with the highest signal intensity occurring at $10 \mu \mathrm{g} / \mathrm{mL}$. By preparing drug concentrations below this value, the linear dynamic range can be reached and limits of detection using the optimized SERS method for codeine can be established. Future work could adapt this method for the detection of codeine and other opiates, due to their structural similarity, in toxicological samples. Codeine, however, should not affect the 
detection of benzodiazepines due to the $\mathrm{pKa}$ of codeine (8.2) being well about the $\mathrm{pH}$ of the optimized SLE method (5) and it should be removed through extraction.

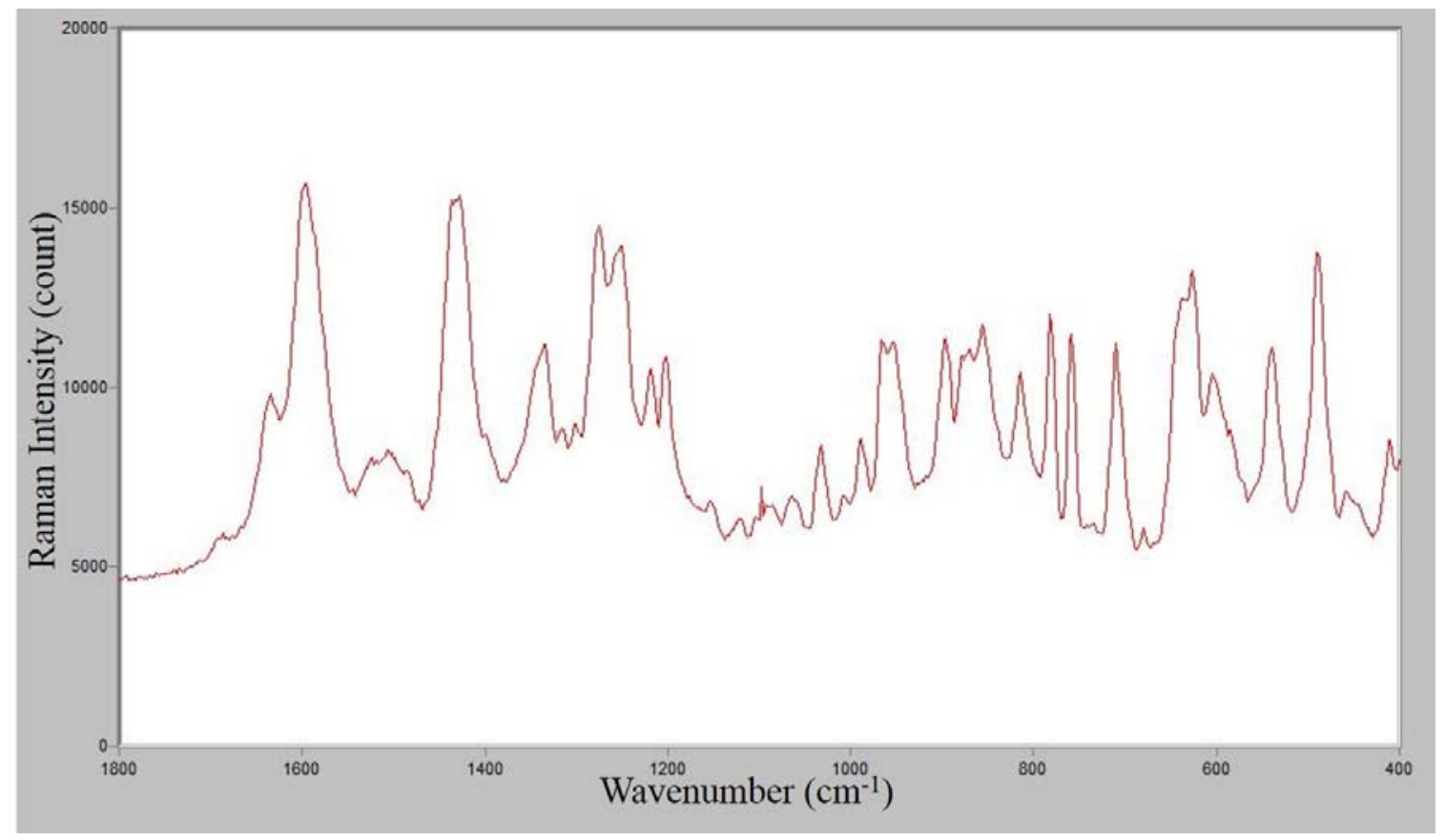

Figure 33: SERS of codeine, $100 \mathrm{ng} / \mathrm{mL}$

$\Delta^{9}$-tetrahydrocannabinol also produced a very strong spectra rich with spectral peaks with intensities ten times those of any of the benzodiazepines previously examined (Figure 34). The peak at $1213 \mathrm{~cm}^{-1}$ was used to measure intensity to prepare a calibration curve. All of the concentrations of THC prepared were above the linear range of the compound, with the highest signal intensity occurring at $10 \mathrm{ng} / \mathrm{mL}$, therefore limits of detection were not determined. This discovery is promising for the analysis of the current problem in forensic science with the detection of the synthetic cannabinoids. The high signal intensities for THC show that the limits of detection for these types of compounds using the optimized SERS method developed here will be very low, in the sub nanogram or high picogram range. The $\mathrm{pKa}$ of $\mathrm{THC}$ is 10.6 , well about the $\mathrm{pH}$ used 
in the optimized extraction method, therefore, the drug should be removed upon extraction.

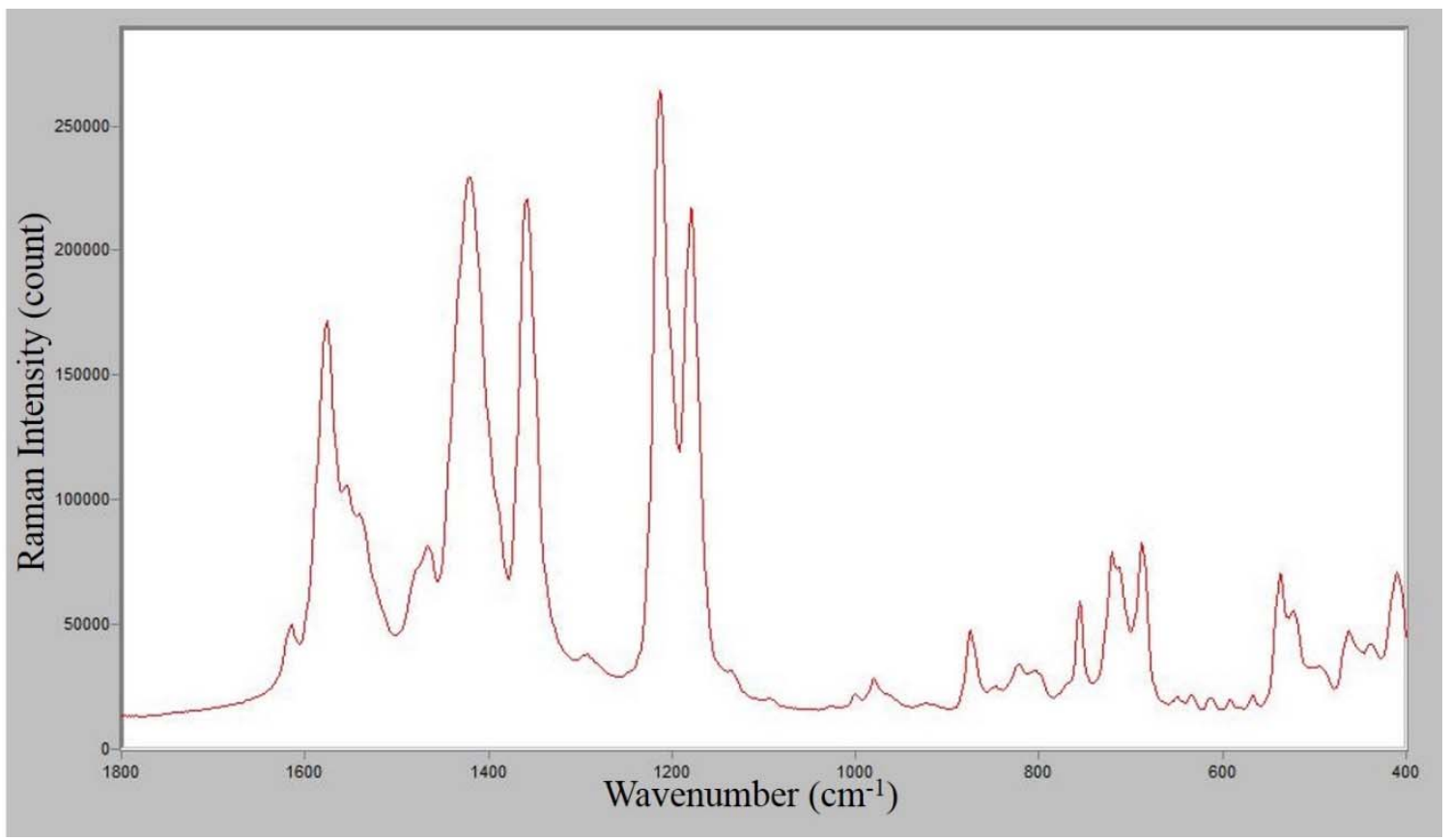

Figure 34: SERS of $\Delta^{9}$-tetrahydrocannabinol, $0.1 \mathrm{ng} / \mathrm{mL}$

\subsubsection{Extraction of 7-amino-flunitrazepam and interference mixtures}

Spiked urine samples were prepared as four different mixtures all including 7amino-flunitrazepam at $250 \mathrm{ng} / \mathrm{mL}$ and a number of interference drugs at the highest biologically relevant concentration listed in Table 6 . The four mixtures were designed in order to identify the interference compound if one did get co-extracted with the benzodiazepine of interest. Each mixture was extracted using the optimized SLE method outlined in Chapter 6.2 and SERS analysis was performed on each extract, which were run in triplicate.

The first mixture contained the interference compounds which produced no SERS spectra as the pure drug, caffeine, GHB, cocaine, salicylic acid, and acetaminophen. The 
second mixture contained nicotine and cotinine due to their similar spectra. The third and fourth mixtures contained codeine and THC respectively. Figure 35 displays the SERS spectra of $250 \mathrm{ng} / \mathrm{mL} 7$-amino-flunitrazepam spiked in urine alone and the extraction results of all four mixtures. It was observed that in all cases the interference compounds tested were either removed from the extraction or if they did co-extract (in the case of those five compounds which did not produce a SERS spectra of the pure drug) did not interfere in the spectra of the benzodiazepine of interest. This shows that this technique can be applied to toxicological urine samples for the extraction, detection, and identification of benzodiazepines without concern for common interferences found in victims of drug facilitated sexual.

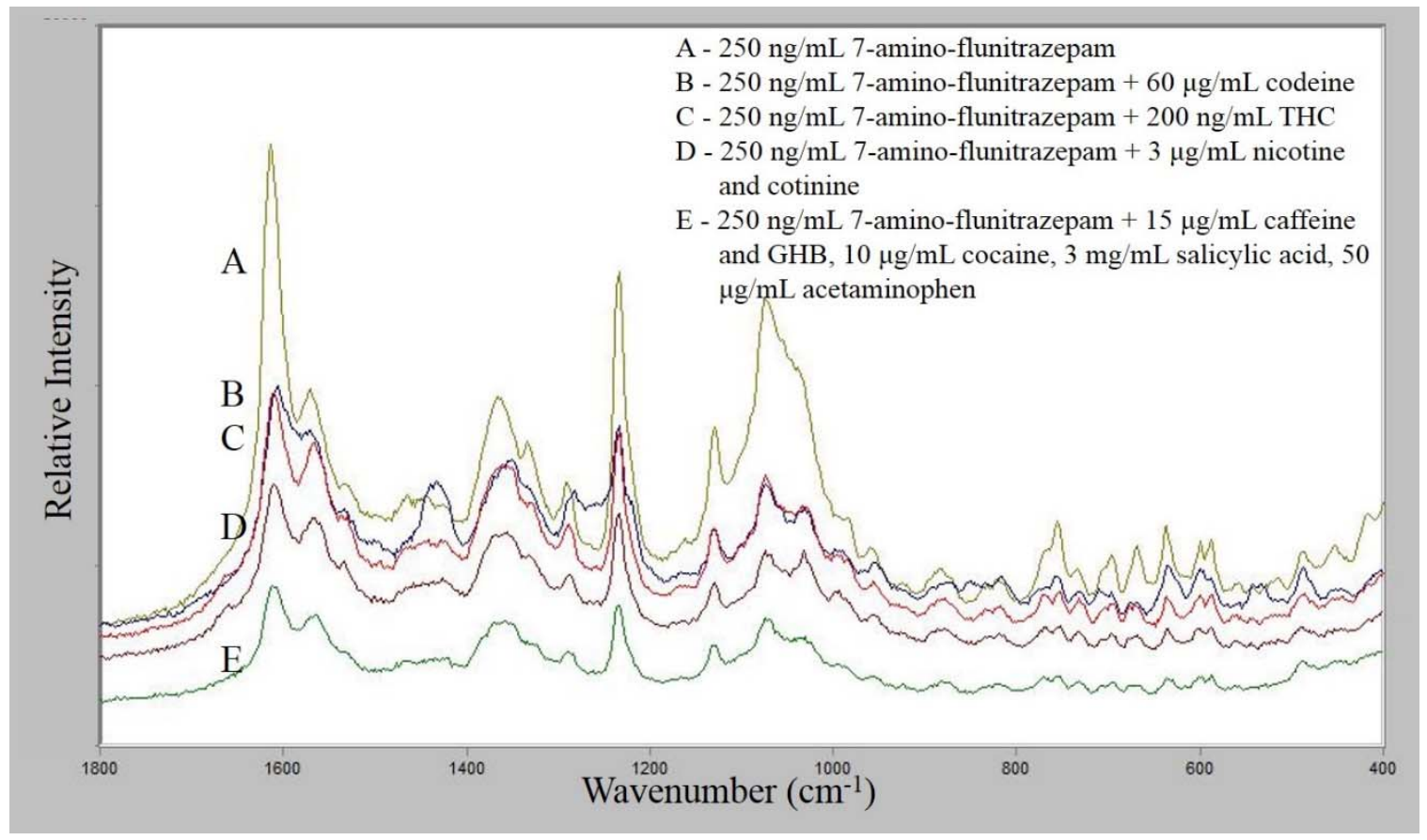

Figure 35: SERS spectra of extraction of interference mixtures as compared to the SERS spectra of extracted 7-amino-flunitrazepam 


\subsection{Conclusions}

Through interference studies it was found that most common drugs that are present in drug facilitated assault case samples do not interfere with the supported liquid extraction, surface-enhanced Raman detection or identification of benzodiazepines. It was also found that the optimized surface-enhanced Raman detection determined previously ${ }^{6}$ can also be applied for the detection of other forensically important compounds such as opiates and synthetic cannabinoids. Overall this work shows the applicability of using this rapid technique, which reduces total analysis time from extraction to detection to under twenty minutes, while minimizing $(1 \mathrm{~mL})$ the amount of biological sample is required without concern for the most common interference compounds. 


\section{VIBRATIONAL SPECTRAL ANALYSIS OF ELEVEN BENZOIDAZEPINES}

\subsection{Introduction}

Raman spectra arise from the various changes in energy from vibrational and electronic transitions of a molecule. The frequency of vibrations can be determined through a quantum treatment, assuming that the transitions in vibrational energy levels are brought about by the absorption of radiation, and that the provided energy exactly matches the difference in the energy levels $(\Delta \mathrm{E})$ between the vibrational quantum states. This occurs when the vibration causes a change in the dipole moment. ${ }^{26}$ Using this treatment, the wavenumber of a given bond in a molecule can be calculated to look at the expected vibrational frequency for a particular bond in a molecule, see Equation 11.

$$
v=\frac{1}{2 \pi c} \sqrt{\frac{k}{\mu}}
$$

Equation 11: where $v$ is the wavenumber of an absorption maximum $\left(\mathrm{cm}^{-1}\right), k$ is the force constant for the bond $(\mathrm{N} / \mathrm{m}), c$ is the velocity of light $\left(\mathrm{cm} \mathrm{s}^{-1}\right)$, and $\mu$ is the reduced mass $(\mathrm{kg})$ defined by Equation $12^{26}$

$$
\mu=\frac{m_{1} m_{2}}{m_{1}+m_{2}}
$$

Equation 12: Reduced mass calculation ${ }^{26}$

It is known that for a given bond, the energy shifts that are observed in a Raman experiment can be compared to those observed in an infrared spectroscopic experiment. If a bond in the molecule is both Raman and infrared active, it is expected that these energies will be identical. Due to this known infrared spectra can be utilized to determine the identity of bands in a Raman spectrum for the same molecule. Also, compounds with 
similar structures may have similar spectral peaks that relate to similar bonds in the molecule. Variation in adjacent bonds can cause these energies to shift slightly between molecules.

In order to establish the peak identities for the individual benzodiazepines, the SERS spectra produced at a concentration of $250 \mathrm{ng} / \mathrm{mL}$ were compared to previously published peak assignments for the Raman spectra of certain drugs. ${ }^{61,44,85}$ Neville and Shurvell have previously provided peak assignments in the Raman spectra of diazepam as well as four other closely related 1,4-benzodiazpines (nordiazepam, pinazepam, halazepam, and prazepam) that were not examined in this study. ${ }^{61}$ Though these compounds were not examined here, the peak assignments can be used to relate other structurally similar benzodiazepines. Raman assignments produced by this group for other benzodiazepines were also used to provide a comprehensive analysis. ${ }^{60,62}$ Peak assignments have also been performed by Trachta, et. al. after SERS analysis. ${ }^{44}$ The specifically examined nordiazepam, diazepam, oxazepam, and flunitrazepam, which were also used in this study, as well as four other benzodiazepines that will not be examined further. ${ }^{44}$ Raman spectral analysis has also been previously performed on the 1,2-triazolo and 1,2-imdazo benzodiazepines. ${ }^{85}$

In the following discussion, structures of the compounds can be referenced in Figure 36 in combination with Table 7. Also, the rings of the structures are labeled in Figure 36 in accordance to how they will be referenced in the discussion and the peak tables. The strength of the bands (very strong $-\mathrm{vs}$, strong $-\mathrm{s}$, moderate $-\mathrm{m}$, weak $-\mathrm{w}$ ), the presence of stretches (str), vibrations (vibr), as well as deformations will be mentioned. 

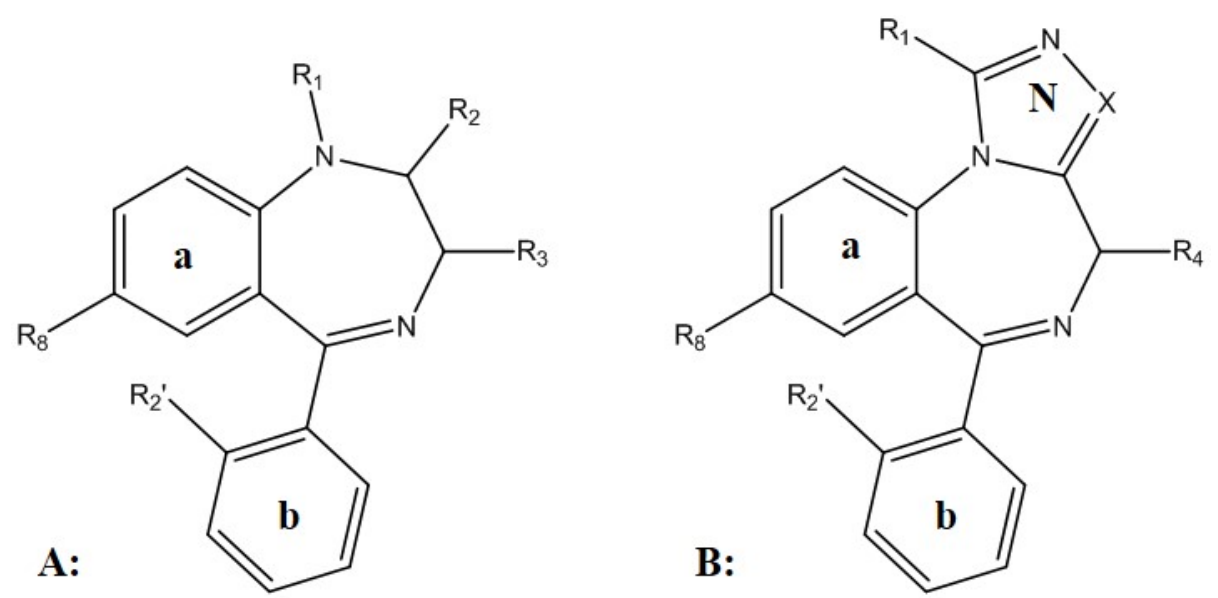

Figure 36: Base structures of (A) 1,4-benzodiazepines and (B) 1,2-triazolo- and 1,2imidazo benzodiazepines ${ }^{6}$

\begin{tabular}{|l|c|c|c|c|c|}
\hline 1,2- Triazolo- and 1,2-imidazo-benzodiazepines & $\mathbf{R}_{\mathbf{1}}$ & $\mathbf{X}$ & $\mathbf{R}_{\mathbf{4}}$ & $\mathbf{R}_{\mathbf{2}}$, & $\mathbf{R}_{\mathbf{8}}$ \\
\hline Alprazolam & $-\mathrm{CH}_{3}$ & $=\mathrm{N}-$ & $-\mathrm{H}$ & $-\mathrm{H}$ & $-\mathrm{Cl}$ \\
\hline Midazolam & $-\mathrm{CH}_{3}$ & $=\mathrm{C}-$ & $-\mathrm{H}$ & $-\mathrm{F}$ & $-\mathrm{Cl}$ \\
\hline Triazolam & $-\mathrm{CH}_{3}$ & $=\mathrm{N}-$ & $-\mathrm{H}$ & $-\mathrm{Cl}$ & $-\mathrm{Cl}$ \\
\hline \multicolumn{1}{|c|}{ 1,4-Benzodiazepines } & $\mathbf{R}_{\mathbf{1}}$ & $\mathbf{R}_{2}$ & $\mathbf{R}_{\mathbf{3}}$ & $\mathbf{R}_{2}$, & $\mathbf{R}_{\mathbf{8}}$ \\
\hline 7-Amino-flunitrazepam (metabolite) & $-\mathrm{CH}_{3}$ & $=\mathrm{O}$ & $-\mathrm{H}$ & $-\mathrm{F}$ & $-\mathrm{NH}_{2}$ \\
\hline Chlordiazepoxide & ---- & $-\mathrm{NHCH}$ & $-\mathrm{H}$ & $-\mathrm{H}$ & $-\mathrm{Cl}$ \\
\hline Clonazepam & $-\mathrm{H}$ & $=\mathrm{O}$ & $-\mathrm{H}$ & $-\mathrm{Cl}$ & $-\mathrm{NO}_{2}$ \\
\hline Diazepam & $-\mathrm{CH}_{3}$ & $=\mathrm{O}$ & $-\mathrm{H}$ & $-\mathrm{H}$ & $-\mathrm{Cl}$ \\
\hline Flunitrazepam & $-\mathrm{CH}_{3}$ & $=\mathrm{O}$ & $-\mathrm{H}$ & $-\mathrm{F}$ & $-\mathrm{NO}_{2}$ \\
\hline Lorazepam & $-\mathrm{H}$ & $=\mathrm{O}$ & $-\mathrm{OH}$ & $-\mathrm{Cl}$ & $-\mathrm{Cl}$ \\
\hline Nordiazepam (metabolite) & $-\mathrm{H}$ & $=\mathrm{O}$ & $-\mathrm{H}$ & $-\mathrm{H}$ & $-\mathrm{Cl}$ \\
\hline Oxazepam (metabolite) & $-\mathrm{H}$ & $=\mathrm{O}$ & $-\mathrm{OH}$ & $-\mathrm{H}$ & $-\mathrm{Cl}$ \\
\hline
\end{tabular}

Table 7: R groups of benzodiazepines ${ }^{6}$ 


\subsection{Analysis of Peaks for All Benzodiazepines and Major Subgroups}

There are two major spectral bands and two minor spectral bands that can be attributed to most benzodiazepines regardless of class. These bands are consistent with the diazepine ring and the interaction between the carbon and nitrogen bonding. There is a strong to very strong peak between $1590-1613 \mathrm{~cm}^{-1}$, due to the aromatic stretch of the carbon nitrogen double bond. Shifting of this peak is caused by the proximity of constituents at the ortho position of the b-ring, which is in free rotation. The second band is medium to very strong in intensity between $1166-1175 \mathrm{~cm}^{-1}$, due to the carbon to carbon to nitrogen stretch. This stretch is also observed by weak bands between $856-$ $889 \mathrm{~cm}^{-1}$. The final weak peak occurs at approximately $820 \mathrm{~cm}^{-1}$ due to the stretch of the bonds on a nitrogen atom between two carbon atoms. Table 8 gives the wavenumbers for each individual benzodiazepines examined in this study for these two prominent peaks. 


\begin{tabular}{|c|c|c|c|c|}
\hline Assignment & Alprazolam & $\begin{array}{c}\text { 7-Amino } \\
\text { Flunitrazepam }\end{array}$ & Chlordiazepoxide & Clonazepam \\
\hline Arom $\mathrm{C}=\mathrm{N}$ str (diazepine ring) & $1593 \mathrm{~s}$ & $1613 \mathrm{vs}$ & $1595 \mathrm{~s}$ & $1606 \mathrm{~m} \mathrm{sh}$ \\
\hline C-C-N str (diazepine ring) & $1166 \mathrm{~m}$ & --------- & $1175 \mathrm{~m}$ & $1168 \mathrm{~m}$ \\
\hline C-C-N str (diazepine ring) & ---------- & $882 \mathrm{w}$ & $863 \mathrm{~m}$ & $889 \mathrm{w}$ \\
\hline $\mathrm{C}-\mathrm{N}-\mathrm{C}$ str (diazepine ring) & ---------- & $820 \mathrm{w}$ & ---------- & ---------- \\
\hline Assignment & Diazepam & Flunitrazepam & Lorazepam & Midazolam \\
\hline Arom $\mathrm{C}=\mathrm{N}$ str (diazepine ring) & 1593 vs & $1594 \mathrm{~m}$ & $1600 \mathrm{~s}$ & 1593 vs \\
\hline C-C-N str (diazepine ring) & $1168 \mathrm{~m}$ & $1168 \mathrm{~m}$ & 1172 vs & $1166 \mathrm{~m}$ \\
\hline $\mathrm{C}-\mathrm{C}-\mathrm{N}$ str (diazepine ring) & $889 \mathrm{w}$ & $885 \mathrm{w}$ & $856 \mathrm{w}$ & --------- \\
\hline $\mathrm{C}-\mathrm{N}-\mathrm{C}$ str (diazepine ring) & $824 \mathrm{w}$ & --------- & --------- & $829 \mathrm{w}$ \\
\hline Assignment & Nordiazepam & Oxazepam & Triazolam & \\
\hline Arom $\mathrm{C}=\mathrm{N}$ str (diazepine ring) & 1599 vs & $1599 \mathrm{~s}$ & 1590 vs & \\
\hline C-C-N str (diazepine ring) & $1167 \mathrm{~s}$ & $1172 \mathrm{~s}$ & $1169 \mathrm{~m}$ & \\
\hline C-C-N str (diazepine ring) & $869 \mathrm{~m}$ & $856 \mathrm{w}$ & ---------- & \\
\hline C-N-C str (diazepine ring) & --------- & $823 \mathrm{w}$ & --------- & \\
\hline
\end{tabular}

Table 8: Observed wavenumbers $\left(\mathrm{cm}^{-1}\right)$ in the surface enhanced Raman spectra of all

$$
\text { benzodiazepines studied } 44,85,86,61
$$

The spectral characterization of the benzodiazepines can be further broken down not only by class but also by the other substituents on the molecule. The 1,2 - triazolo and imidazo benzodiazepines specifically have spectral peaks due to the presence of the additional nitrogen containing ring (N-ring) attached at the 1,2 positions on the diazepine ring. The 1,2-triazolo-benzodiazpines alprazolam and triazolam have two weak to medium ring "breathing" peaks at $1257 \mathrm{~cm}^{-1}$ and $1138 \mathrm{~cm}^{-1}$ attributed to the N-ring containing three nitrogen atoms. Midazolam, a 1,2- imidazo-benzodizepine which only has two nitrogen atoms in the N-ring, has both a weak peak attributed to ring "breathing" 
$\left(1137 \mathrm{~cm}^{-1}\right)$ as well as a medium peak at $1305 \mathrm{~cm}^{-1}$ due to the amide III band. Specific wavenumbers are shown in Table 9.

\begin{tabular}{|l||c|c|c|}
\hline \multicolumn{1}{|c||}{ Assignment } & Alprazolam & Midazolam & Triazolam \\
\hline \hline Amide III band (N-ring) & -------- & $1305 \mathrm{~m}$ & -------- \\
\hline Ring “breathing” (N-ring) & $1256 \mathrm{w}$ & -------- & $1258 \mathrm{w}$ \\
\hline Ring “breathing” (N-ring) & $1137 \mathrm{~m}$ & $1137 \mathrm{w}$ & $1139 \mathrm{w}$ \\
\hline
\end{tabular}

Table 9: Observed wavenumbers $\left(\mathrm{cm}^{-1}\right)$ in the surface enhanced Raman spectra of 1,2-

$$
\text { triazolo and imidazo benzodiazepines studied }{ }^{85}
$$

The benzene ring in the base benzodiazepine structure indicated as the b-ring in Figure 36 can be monosubstituted disubstituted at the ortho position. While the disubstitution shows spectral bands in the infrared spectra, ${ }^{61,44,85,86}$ these bands are not prevalent in the Raman spectra. Generally, two spectral peaks are observed, however, that can be attributed to the monosubstituted b-ring. There is a peak due to the vibration of the benzene ring with the carbon of the diazepine ring at $1205 \mathrm{~cm}^{-1}$ as well as a medium peak assigned to the monosubstituted ring "breathing" at approximately $998 \mathrm{~cm}^{-}$

1. The benzodiazepines which have the monosubstituted b-ring as well as their specific wavenumber assignments are in Table 10.

\begin{tabular}{|l||c|c|c|c|c|}
\hline \multicolumn{1}{|c||}{ Assignment } & Alprazolam & Chlordiazepoxide & Diazepam & Nordiazepam & Oxazepam \\
\hline \hline C6 $\mathrm{H}_{5}$-C vibr. & $1207 \mathrm{~m}$ & --------- & $1207 \mathrm{w}$ & $1204 \mathrm{~m}$ & -------- \\
\hline $\begin{array}{l}\text { Monosubstituted } \\
\text { "breathing" }\end{array}$ & $1001 \mathrm{~m}$ & $1001 \mathrm{~m}$ & $996 \mathrm{~m}$ & $993 \mathrm{~m}$ & $996 \mathrm{~m}$ \\
\hline \hline
\end{tabular}

Table 10: Observed wavenumbers $\left(\mathrm{cm}^{-1}\right)$ in the surface enhanced Raman spectra of monosubstituted benzene b-ring

Shared spectral characteristics can also be observed across the benzodiazepines based on single structural features. These bands can help in further classifying the 
benzodiazepines based on structural similarities. A weak (alprazolam) to very strong (flunitrazepam) peak between $1344-1402 \mathrm{~cm}^{-1}$ is observed in five of the benzodiazepines studied due to the stretch of the bond between the nitrogen in the diazepine ring and a methyl group. Six benzodiazepines display a weak peak at approximately $671 \mathrm{~cm}^{-1}$ due to the deformation of the carbon oxygen double bond on the diazepine ring.

\begin{tabular}{|c|l|}
\hline Wavenumber & \multicolumn{1}{|c|}{ Benzodiazepine } \\
\hline \hline $1378 \mathrm{w}$ & Alprazolam \\
\hline $1371 \mathrm{~m}$ & 7 -Amino-Flunitrazepam \\
\hline $1378 \mathrm{~m}$ & Chlordiazepoxide \\
\hline $1404 \mathrm{~m}$ & Diazepam \\
\hline $1344 \mathrm{vs}$ & Flunitrazepam \\
\hline
\end{tabular}

Table 11: Observed wavenumbers $\left(\mathrm{cm}^{-1}\right)$ in the surface enhanced Raman spectra of N$\mathrm{CH}_{3}$ stretch

\begin{tabular}{|c|l|}
\hline Wavenumber & \multicolumn{1}{|c|}{ Benzodiazepine } \\
\hline \hline $670 \mathrm{w}$ & 7 -Amino-Flunitrazepam \\
\hline $676 \mathrm{w}$ & Clonazepam \\
\hline $689 \mathrm{~m}$ & Diazepam \\
\hline $670 \mathrm{w}$ & Flunitrazepam \\
\hline $671 \mathrm{w}$ & Lorazepam \\
\hline $671 \mathrm{w}$ & Nordiazepam \\
\hline
\end{tabular}

Table 12: Observed wavenumbers $\left(\mathrm{cm}^{-1}\right)$ in the surface enhanced Raman spectra of $\mathrm{C}=\mathrm{O}$ deformation on diazepine ring

Many benzodiazepines have halogen atoms added to their structure in order to improve their physiological activity. Halogens bonded to carbon atoms show specific spectral peaks. Those benzodiazepines that have chlorine atoms, regardless of being 
attached on the b,a, or both benzene rings, show a weak spectral band at approximately $590 \mathrm{~cm}^{-1}$. Those that have a fluorine atom show a medium to strong spectral band at approximately $1220 \mathrm{~cm}^{-1}$. These halogen bands are generally weak and can be dwarfed by other components of the spectra or are so weak they are not above the baseline of the spectra. The later occurs in the cases of where chlorine atoms are present in the molecule but no peak is observed (clonazepam, lorazepam, oxazepam). ${ }^{86}$ The former occurs for flunitrazepam, where the broad band at $1344 \mathrm{~cm}^{-1}$, masks any possible peak at $1220 \mathrm{~cm}^{-1}$ due to the fluorine carbon stretch.

\begin{tabular}{|c|l|}
\hline Wavenumber & Benzodiazepine \\
\hline \hline $589 \mathrm{w}$ & Alprazolam \\
\hline $573 \mathrm{w}$ & Chlordiazepoxide \\
\hline$------*$ & Clonazepam \\
\hline $593 \mathrm{w}$ & Diazepam \\
\hline$------*$ & Lorazepam \\
\hline $590 \mathrm{w}$ & Midazolam \\
\hline$-----*^{*}$ & Oxazepam \\
\hline $587 \mathrm{w}$ & Nordiazepam \\
\hline $590 \mathrm{w}$ & Triazolam \\
\hline
\end{tabular}

Table 13: Observed wavenumbers $\left(\mathrm{cm}^{-1}\right)$ in the surface enhanced Raman spectra of C-Cl stretching 


\begin{tabular}{|c|l|}
\hline Wavenumber & \multicolumn{1}{|c|}{ Benzodiazepine } \\
\hline \hline $1233 \mathrm{~s}$ & 7-Amino-Flunitrazepam \\
\hline$------*$ & Flunitrazepam \\
\hline $1211 \mathrm{~m}$ & Midazolam \\
\hline
\end{tabular}

Table 14: Observed wavenumbers $\left(\mathrm{cm}^{-1}\right)$ in the surface enhanced Raman Spectra of C-F stretching

\subsection{Additional Spectral Analysis of Individual Benzodiazepines}

Each benzodiazepine can be characterized by the individualizing combination of peaks previously mentioned as well as additional peaks observed in the following tables. The relative intensity of these peaks in relation to the other peaks in the spectra as well as their wavenumbers produce a spectral fingerprint for each benzodiazepine. The following pages will show the surface enhanced Raman spectra of each benzodiazepine as well as a table outlining the wavenumbers, relative intensities, and assignments for each spectral peak. Strong identifying peaks that were not previously characterized will also be discussed in the case of some compounds.

\subsubsection{Alprazolam}

Alprazolam is the most basic of the 1,2-triazolo-benzodiazpines. There are no additional identifying peaks. 


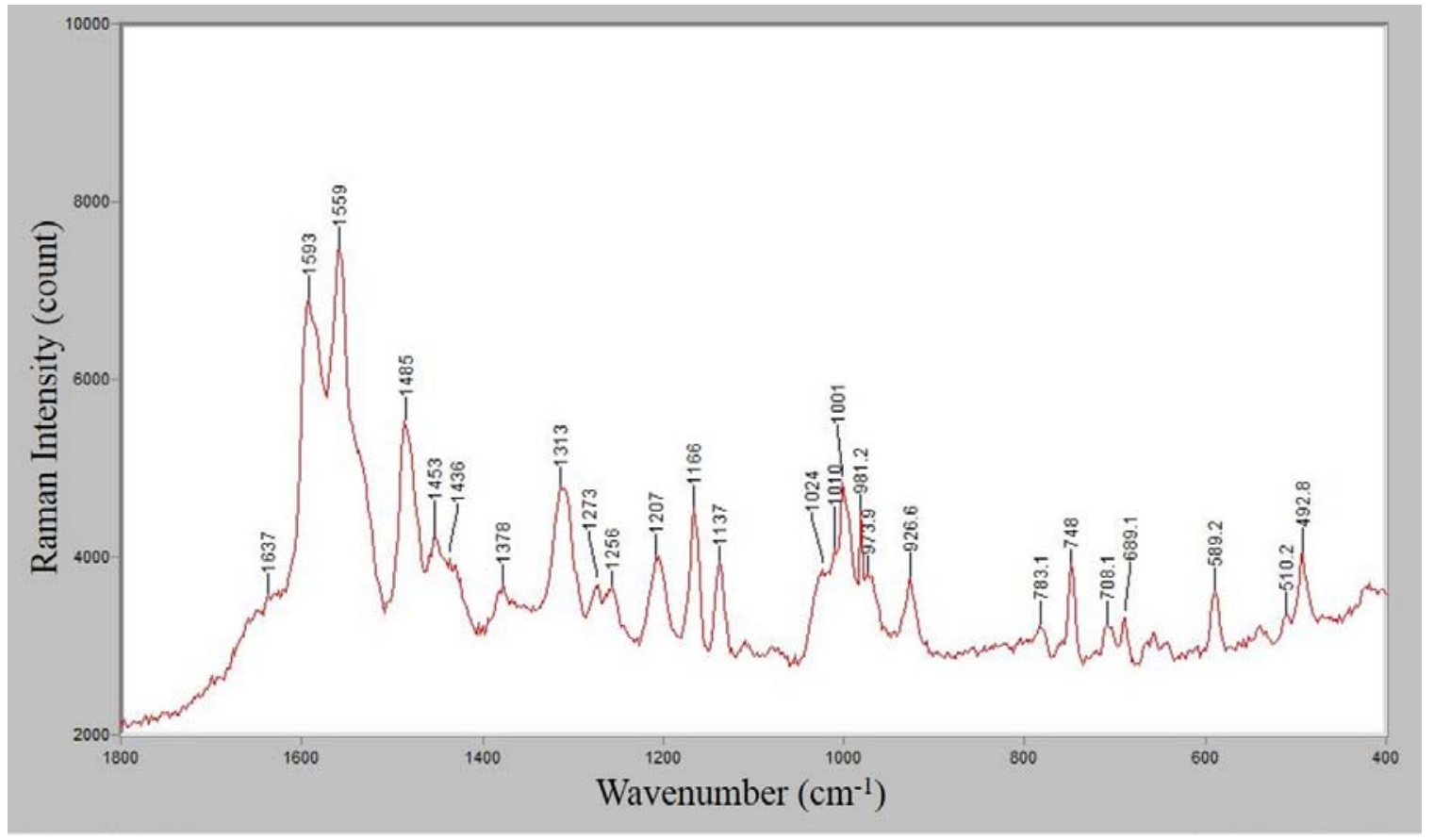

Figure 37: SERS spectra alprazolam

\begin{tabular}{|c|l|c|l|}
\hline Wavenumber & \multicolumn{1}{|c|}{ Assignment } & Wavenumber & \multicolumn{1}{c|}{ Assignment } \\
\hline $1593 \mathrm{~s}$ & Arom C=N str (diazepine ring) & $1166 \mathrm{~m}$ & C-C-N str (diazepine ring) \\
$1559 \mathrm{~s}$ & Arom C=C str (a-ring) & $1137 \mathrm{~m}$ & Ring breathing (N-ring) \\
$1485 \mathrm{~m}$ & Arom C=C str (a-ring) & $1001 \mathrm{~m}$ & Monosubstituted "breathing" (b-ring) \\
$1378 \mathrm{w}$ & $\mathrm{CH}$ str (N-ring) & $927 \mathrm{~m}$ & CH deformation \\
$1313 \mathrm{~m}$ & $\mathrm{C}=\mathrm{N}-\mathrm{C}$ sym. str (diazepine ring) & $783 \mathrm{w}$ & Ring vibration (b-ring) \\
$1273 \mathrm{w}$ & In plane CH def (a-ring) & $708 \mathrm{w}$ & ----------------------- \\
$1256 \mathrm{w}$ & Ring "breathing" & $689 \mathrm{w}$ & Out-of-plane CH deformation (b-ring) \\
$1207 \mathrm{~m}$ & C6 $\mathrm{H}_{5}-\mathrm{C}$ vibr. (b-ring) & $589 \mathrm{w}$ & C-Cl str (a-ring) \\
\hline
\end{tabular}

Table 15: Observed wavenumbers $\left(\mathrm{cm}^{-1}\right)$ in the surface enhanced Raman spectra of

$$
\operatorname{alprazolam}^{85}
$$

\subsubsection{7-Amino-flunitrazepam}

7-Amino-flunitrazepam is the major metabolite of flunitrazepam. An important additional identifying peak is the asymmetric $\mathrm{NH}_{2}$ stretch at $1571 \mathrm{~cm}^{-1}$. 


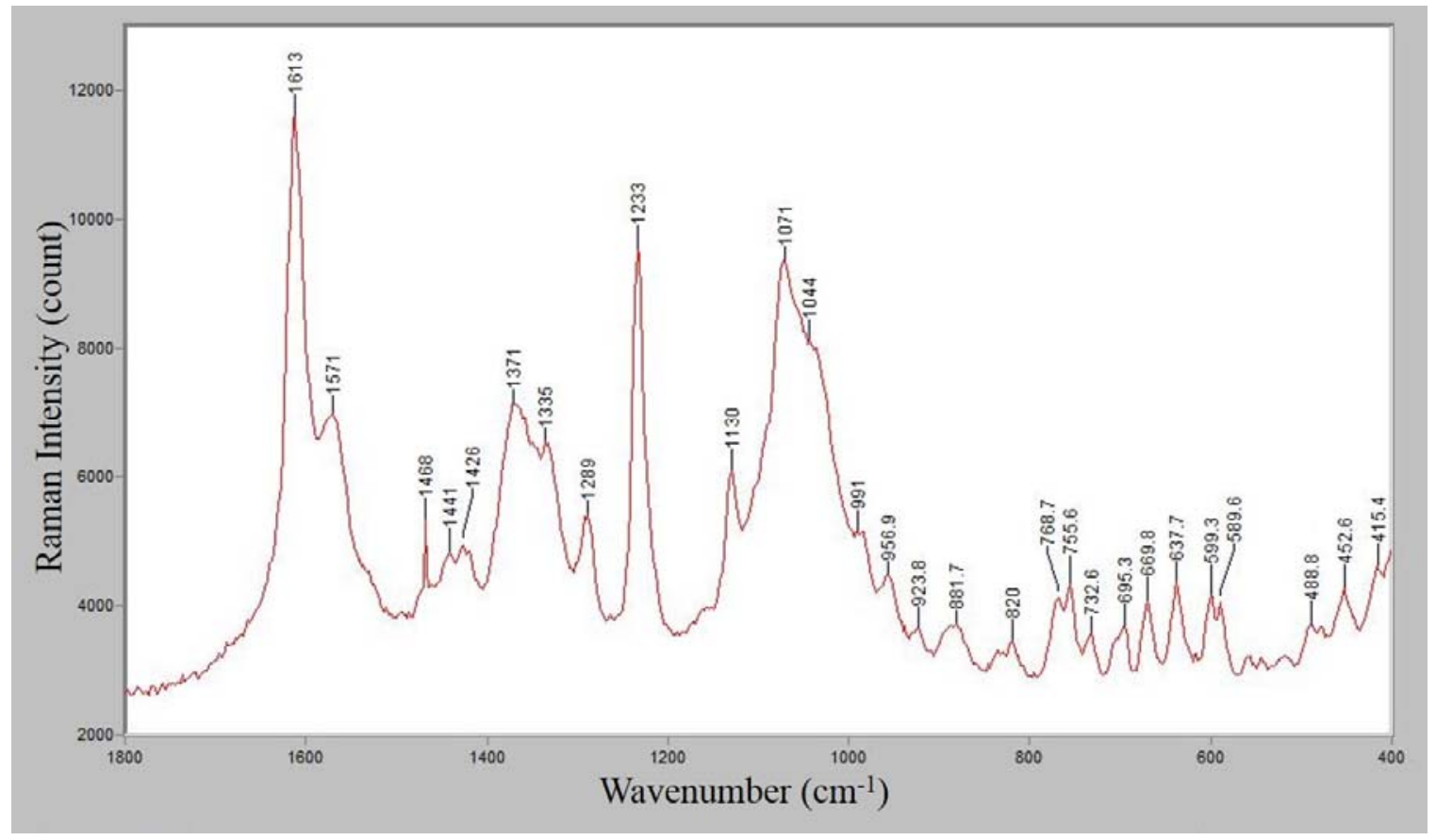

Figure 38: SERS spectra 7-amino-flunitrazepam

\begin{tabular}{|c|c|c|c|}
\hline Wavenumber & Assignment & Wavenumber & Assignment \\
\hline 1613 vs & Arom $\mathrm{C}=\mathrm{N}$ str (diazepine ring) & $924 \mathrm{w}$ & $\mathrm{C}-\mathrm{H}$ deformation \\
\hline $1571 \mathrm{~m}$ & Asymmetric $\mathrm{NH}_{2}$ str & $882 \mathrm{w}$ & C-C-N str (diazepine ring) \\
\hline $1441 \mathrm{w}$ & Arom $\mathrm{C}=\mathrm{C}$ str (a-ring) & $820 \mathrm{w}$ & C-N-C str (diazepine ring) \\
\hline $1426 \mathrm{w}$ & Asymmetric $\mathrm{CH}_{3}$ deformation & $769 \mathrm{~m} \mathrm{sh}$ & 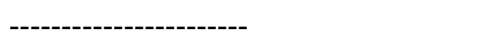 \\
\hline $1371 \mathrm{~m}$ & $\mathrm{~N}-\mathrm{CH}_{3}$ str (diazepine ring) & $756 \mathrm{~m}$ & $\mathrm{C}-\mathrm{H}$ out of plane deformation \\
\hline $1335 \mathrm{~m} \mathrm{sh}$ & $\begin{array}{l}\mathrm{C}-\mathrm{C} \text { in plane def (a-, diazepine } \\
\text { rings) }\end{array}$ & $733 \mathrm{w}$ & ----------------------- \\
\hline $1289 \mathrm{~m}$ & In plane $\mathrm{CH}$ def (a-ring) & $695 \mathrm{w}$ & Out-of-plane $\mathrm{CH}$ deformation (b-ring) \\
\hline $1233 \mathrm{~s}$ & C-F str (b-ring) & $670 \mathrm{w}$ & $\mathrm{C}=\mathrm{O}$ deformation \\
\hline $1130 \mathrm{~m}$ & $\begin{array}{l}\mathrm{C}-\mathrm{H} \text { in plane deformations (a-, } \\
\text { diazepine ring) }\end{array}$ & $638 \mathrm{w}$ & Out-of-plane deformation \\
\hline $1070 \mathrm{~s}$ & $\mathrm{C}-\mathrm{H}$ deformation & $599 \mathrm{w}$ & Ring "breathing" (a,b rings) \\
\hline
\end{tabular}

Table 16: Observed wavenumbers $\left(\mathrm{cm}^{-1}\right)$ in the surface enhanced Raman spectra of 7amino-flunitrazepam ${ }^{85-86,61,44}$ 


\subsubsection{Chlordiazepoxide}

Chlordiazepoxide was the first benzodiazepine synthesized and falls into the 1,4benzodiazepine class. Aside from previously mentioned peaks, the combination of strong peaks at 1595,1544 , and $1481 \mathrm{~cm}^{-1}$ is characteristic of this compound.

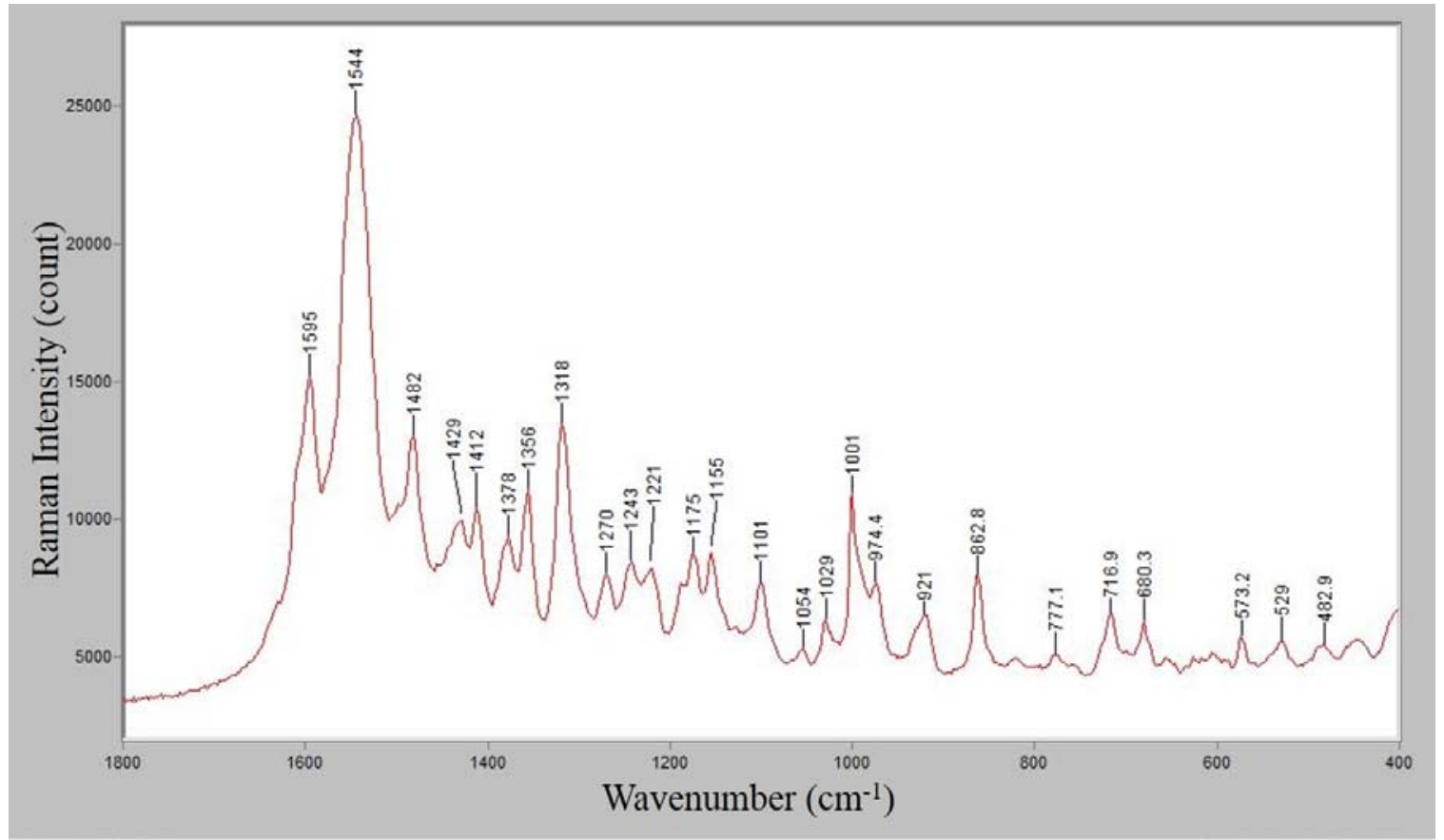

Figure 39: SERS spectra chlordiazepoxide 


\begin{tabular}{|c|c|c|c|}
\hline Wavenumber & Assignment & Wavenumber & Assignment \\
\hline $1595 \mathrm{~s}$ & Arom $\mathrm{C}=\mathrm{N}$ str (diazepine ring) & $1155 \mathrm{~m}$ & 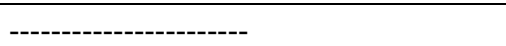 \\
\hline 1544 vs & Arom $\mathrm{C}=\mathrm{C}$ str (a-ring) & $1100 \mathrm{w}$ & $\begin{array}{l}\mathrm{C}-\mathrm{H} \text { in plane deformation (a, diazepine } \\
\text { rings) }\end{array}$ \\
\hline $1481 \mathrm{~s}$ & Arom $\mathrm{C}=\mathrm{C}$ str (a-ring) & $1029 \mathrm{w}$ & $\mathrm{C}-\mathrm{H}$ in plane deformation (b-ring) \\
\hline $1428 \mathrm{~m} \mathrm{sh}$ & Asymmetric $\mathrm{CH}_{3}$ deformation & $1001 \mathrm{~m}$ & Monosubstituted "breathing" (b-ring) \\
\hline $1412 \mathrm{~s}$ & ------------------------ & $974 \mathrm{w}$ sh & ------------------------ \\
\hline $1378 \mathrm{~m}$ & $\mathrm{~N}-\mathrm{CH}_{3} \operatorname{str}$ (diazepine ring) & $921 \mathrm{w}$ & C-H deformation \\
\hline 1356 & ------------------------- & $863 \mathrm{~m}$ & $\mathrm{C}-\mathrm{C}-\mathrm{N}$ str (diazepine ring) \\
\hline $1318 \mathrm{~s}$ & $\mathrm{C}=\mathrm{N}-\mathrm{C}$ sym. $\operatorname{str}$ (diazepine ring) & $716 \mathrm{w}$ & ----------------------- \\
\hline $1270 \mathrm{w}$ & $\begin{array}{l}\mathrm{C}-\mathrm{H} \text { in plane deformation (a- } \\
\text { ring) }\end{array}$ & $680 \mathrm{w}$ & Out-of-plane deformation \\
\hline $1175 \mathrm{~m}$ & C-C-N str (diazepine ring) & $573 \mathrm{w}$ & $\mathrm{C}-\mathrm{Cl}$ str (a-ring) \\
\hline
\end{tabular}

Table 17: Observed wavenumbers $\left(\mathrm{cm}^{-1}\right)$ in the surface enhanced Raman spectra of

$$
\text { chlordiazepoxide }{ }^{44,61}
$$

\subsubsection{Clonazepam}

Clonazepam is a 1,4-benzodiazpine that has two additional peaks which correspond to the amino group on the diazepine ring. These peaks include a medium intensity peak at $1569 \mathrm{~cm}^{-1}$ corresponding to the asymmetric $\mathrm{NO}_{2}$ stretch and a weak peak at $560 \mathrm{~cm}^{-1}$ due to the deformation of the functional group. 


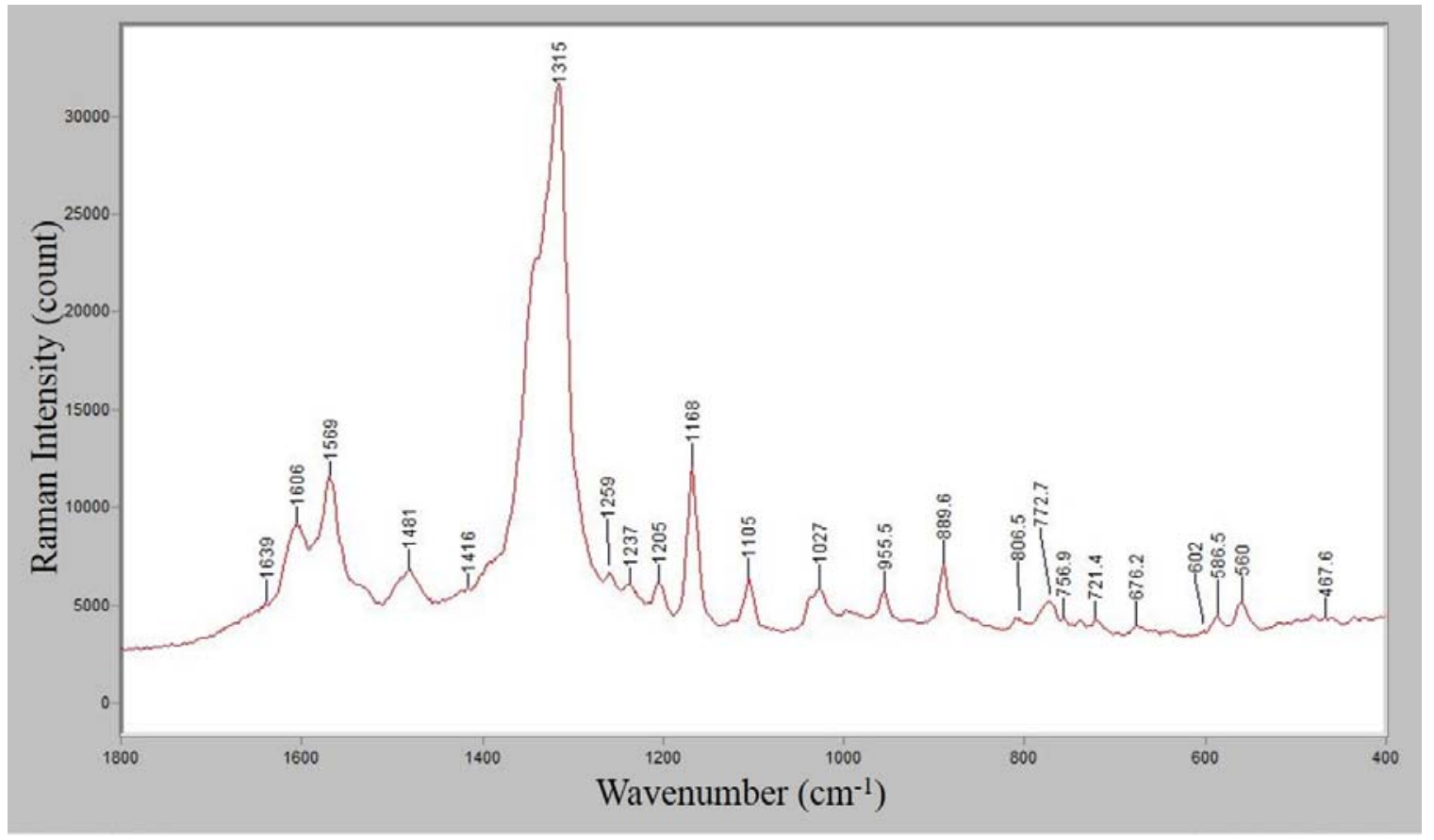

Figure 40: SERS spectra clonazepam

\begin{tabular}{|c|c|c|c|}
\hline Wavenumber & Assignment & Wavenumber & Assignment \\
\hline $1606 \mathrm{~m} \mathrm{sh}$ & Arom $\mathrm{C}=\mathrm{N}$ str (diazepine ring) & $1105 \mathrm{w}$ & $\begin{array}{l}\mathrm{C}-\mathrm{H} \text { in plane deformation (a, diazepine } \\
\text { rings) }\end{array}$ \\
\hline $1569 \mathrm{~m}$ & Asymmetric $\mathrm{NO}_{2}$ str & $1027 \mathrm{w}$ & $\mathrm{C}-\mathrm{H}$ in plane deformation (b-ring) \\
\hline $1480 \mathrm{w}$ & $\begin{array}{l}\mathrm{C}-\mathrm{H} \text { in plane deformation (a- } \\
\text { ring) }\end{array}$ & $956 \mathrm{w}$ & $\mathrm{C}-\mathrm{H}$ in plane deformation (b-ring) \\
\hline 1315 vs & $\mathrm{C}=\mathrm{N}-\mathrm{C}$ sym. str (diazepine ring) & $889 \mathrm{w}$ & C-C-N str (diazepine ring) \\
\hline $1259 \mathrm{w}$ & Arom $\mathrm{C}=\mathrm{C}$ str & $772 \mathrm{w}$ & $\mathrm{C}-\mathrm{H}$ out of plane deformation \\
\hline $1237 \mathrm{w}$ & $\mathrm{CH}_{2}$ wag & $757 \mathrm{w}$ & $\mathrm{C}-\mathrm{H}$ out of plane deformation \\
\hline $1205 \mathrm{w}$ & $\begin{array}{l}\mathrm{C}-\mathrm{H} \text { in plane deformation (a- } \\
\text { ring) }\end{array}$ & $676 \mathrm{w}$ & $\mathrm{C}=\mathrm{O}$ deformation \\
\hline $1168 \mathrm{~m}$ & C-C-N str (diazepine ring) & $560 \mathrm{w}$ & $\mathrm{NO}_{2}$ deformation \\
\hline
\end{tabular}

Table 18: Observed wavenumbers $\left(\mathrm{cm}^{-1}\right)$ in the surface enhanced Raman spectra of clonazepam $^{61}$

\subsubsection{Diazepam}

Diazepam is the most basic of the 1,4-benzodiazpines. There are no additional identifying peaks. 


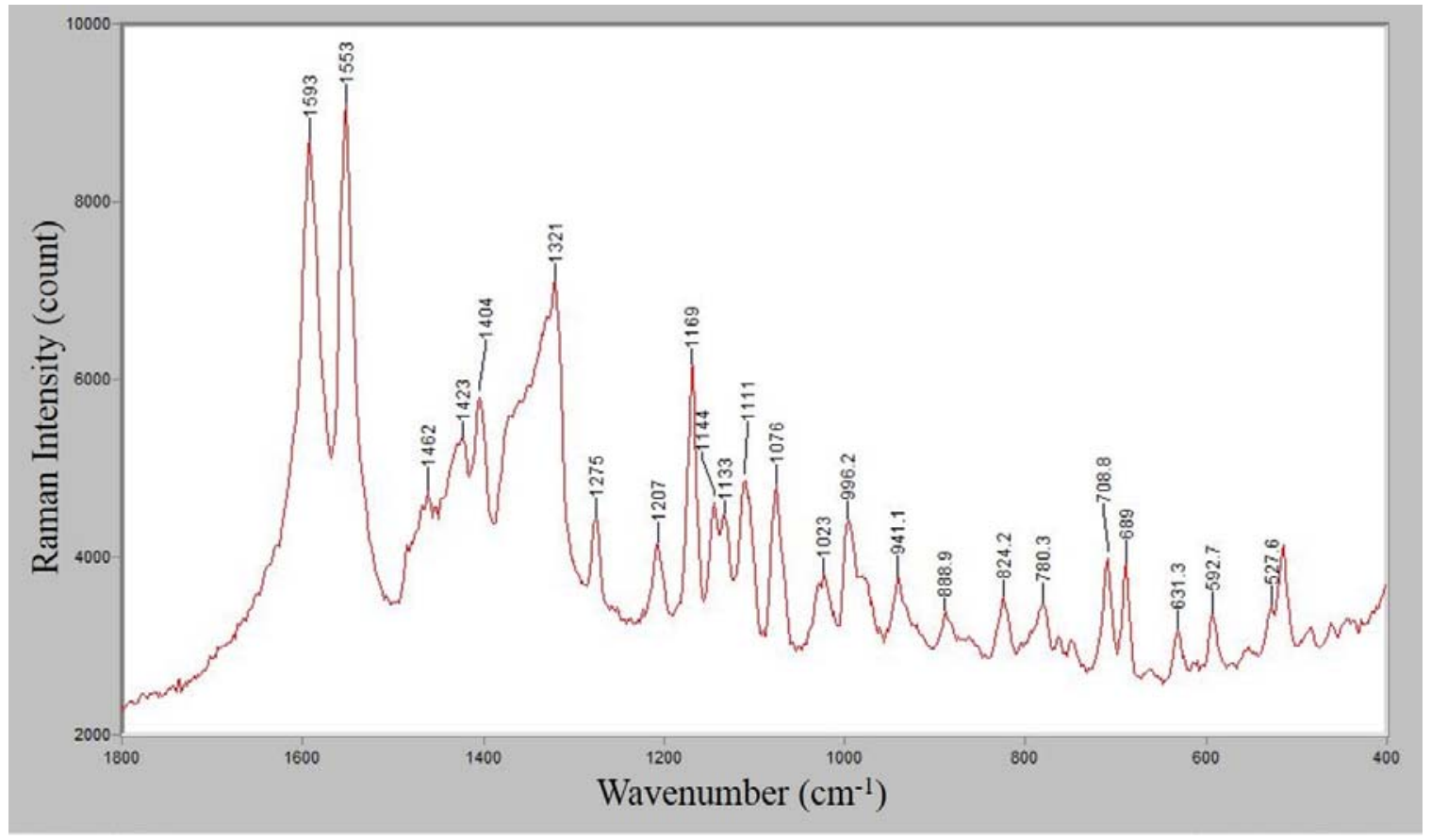

Figure 41: SERS spectra diazepam

\begin{tabular}{|c|c|c|c|}
\hline Wavenumber & Assignment & Wavenumber & Assignment \\
\hline 1593 vs & Arom $\mathrm{C}=\mathrm{N}$ str (diazepine ring) & $1076 \mathrm{~m}$ & $\begin{array}{l}\mathrm{C}-\mathrm{C} \text { in plane deformation }(\mathrm{a}, \mathrm{b} \\
\text { diazepine rings) }\end{array}$ \\
\hline 1553 vs & Arom $\mathrm{C}=\mathrm{C}$ str (a-ring) & $1023 \mathrm{w}$ & $\mathrm{C}-\mathrm{H}$ in plane deformation (b-ring) \\
\hline $1423 \mathrm{w}$ & $\begin{array}{l}\text { Asymmetric } \mathrm{CH}_{3} \text { deformation (a- } \\
\text { ring) }\end{array}$ & $996 \mathrm{~m}$ & Monosubstituted "breathing" (b-ring) \\
\hline $1404 \mathrm{~m}$ & $\mathrm{~N}-\mathrm{CH}_{3}$ str (diazepine ring) & $889 \mathrm{w}$ & C-C-N str (diazepine ring) \\
\hline $1321 \mathrm{~s}$ & $\begin{array}{l}\mathrm{C}-\mathrm{H} \text { in plane deformation (a- } \\
\text { ring) }\end{array}$ & $824 \mathrm{w}$ & C-N-C str (diazepine ring) \\
\hline $1275 \mathrm{w}$ & $\begin{array}{l}\mathrm{C}-\mathrm{C} \text { in plane deformation (a- } \\
\text {,b,diazpeine ring) }\end{array}$ & $780 \mathrm{w}$ & $\mathrm{C}-\mathrm{H}$ out of plane deformation \\
\hline $1207 \mathrm{w}$ & $\mathrm{C}_{6} \mathrm{H}_{5}-\mathrm{C}$ vibr. (b-ring) & $709 \mathrm{~m}$ & $\mathrm{C}-\mathrm{H}$ out of plane deformation (b-ring) \\
\hline $1168 \mathrm{~m}$ & C-C-N str (diazepine ring) & $689 \mathrm{~m}$ & $\mathrm{C}=\mathrm{O}$ deformation \\
\hline $1144 \mathrm{~m}$ & $\mathrm{CH}_{3}$ rocking & $631 \mathrm{w}$ & Ring "breathing" \\
\hline $1133 \mathrm{w}$ sh & $\begin{array}{l}\mathrm{C}-\mathrm{H} \text { in plane deformation (a- } \\
\text { ring) }\end{array}$ & $593 \mathrm{w}$ & $\mathrm{C}-\mathrm{Cl}$ str \\
\hline $1111 \mathrm{~m}$ & $\begin{array}{l}\mathrm{C}-\mathrm{H} \text { in plane deformation ( } \mathrm{a}, \\
\text { diazepine rings) }\end{array}$ & $527 \mathrm{w}$ & Out of plane deformation \\
\hline
\end{tabular}

Table 19: Observed wavenumbers $\left(\mathrm{cm}^{-1}\right)$ in the surface enhanced Raman spectra of

$$
\text { diazepam }^{61,44}
$$




\subsubsection{Flunitrazepam}

Flunitrazepam is a 1,4-benzodiazepine whose spectra can be characterized by the very prominent peak at $1344 \mathrm{~cm}^{-1}$ which causes all the other spectral peaks to appear weak by comparison. In addition there is a band at $1568 \mathrm{~cm}^{-1}$ due to the asymmetric $\mathrm{NO}_{2}$ stretch.

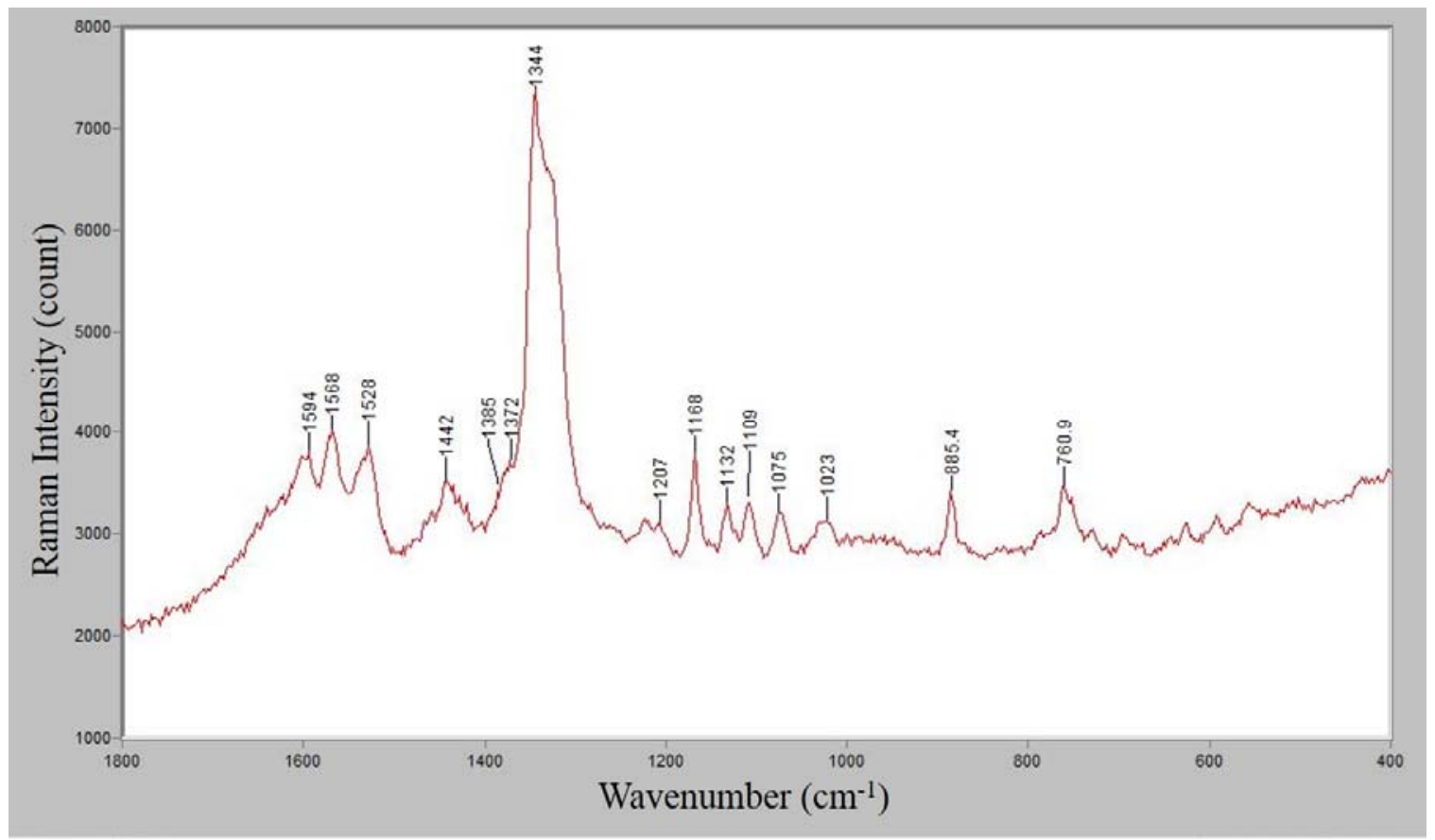

Figure 42: SERS spectra flunitrazepam

\begin{tabular}{|c|c|c|c|}
\hline Wavenumber & Assignment & Wavenumber & Assignment \\
\hline $1594 \mathrm{~m}$ & Arom $\mathrm{C}=\mathrm{N}$ str (diazepine ring) & $1132 \mathrm{w}$ & $\mathrm{CH}_{3}$ deformation \\
\hline $1568 \mathrm{w}$ & Asymmetric $\mathrm{NO}_{2}$ str & $1109 \mathrm{w}$ & $\begin{array}{l}\mathrm{C}-\mathrm{H} \text { in plane deformation (a,diazepine } \\
\text { ring) }\end{array}$ \\
\hline $1528 \mathrm{w}$ & Arom $\mathrm{C}=\mathrm{C}$ str (a-ring) & $1075 \mathrm{w}$ & $\mathrm{C}-\mathrm{C}$ deformation ( $\mathrm{a}, \mathrm{b}$, diazepine rings) \\
\hline $1442 \mathrm{w}$ & $\begin{array}{l}\mathrm{C}-\mathrm{H} \text { in plane deformation (b- } \\
\text { ring) }\end{array}$ & $885 \mathrm{w}$ & C-C-N str (diazepine ring) \\
\hline $1344 \mathrm{vs}$ & $\mathrm{N}-\mathrm{CH}_{3}$ str (diazepine ring) & $760 \mathrm{w}$ & $\mathrm{C}-\mathrm{H}$ out of plane deformation \\
\hline $1168 \mathrm{~m}$ & $\mathrm{C}-\mathrm{C}-\mathrm{N}$ str (diazepine ring) & $670 \mathrm{w}$ & $\mathrm{C}=\mathrm{O}$ deformation \\
\hline
\end{tabular}

Table 20: Observed wavenumbers $\left(\mathrm{cm}^{-1}\right)$ in the surface enhanced Raman spectra of

$$
\text { flunitrazepam }{ }^{44,61,86}
$$




\subsubsection{Lorazepam}

Lorazepam is a 1,4-benzodiazpine that has no additional identifying peaks not previously discussed.

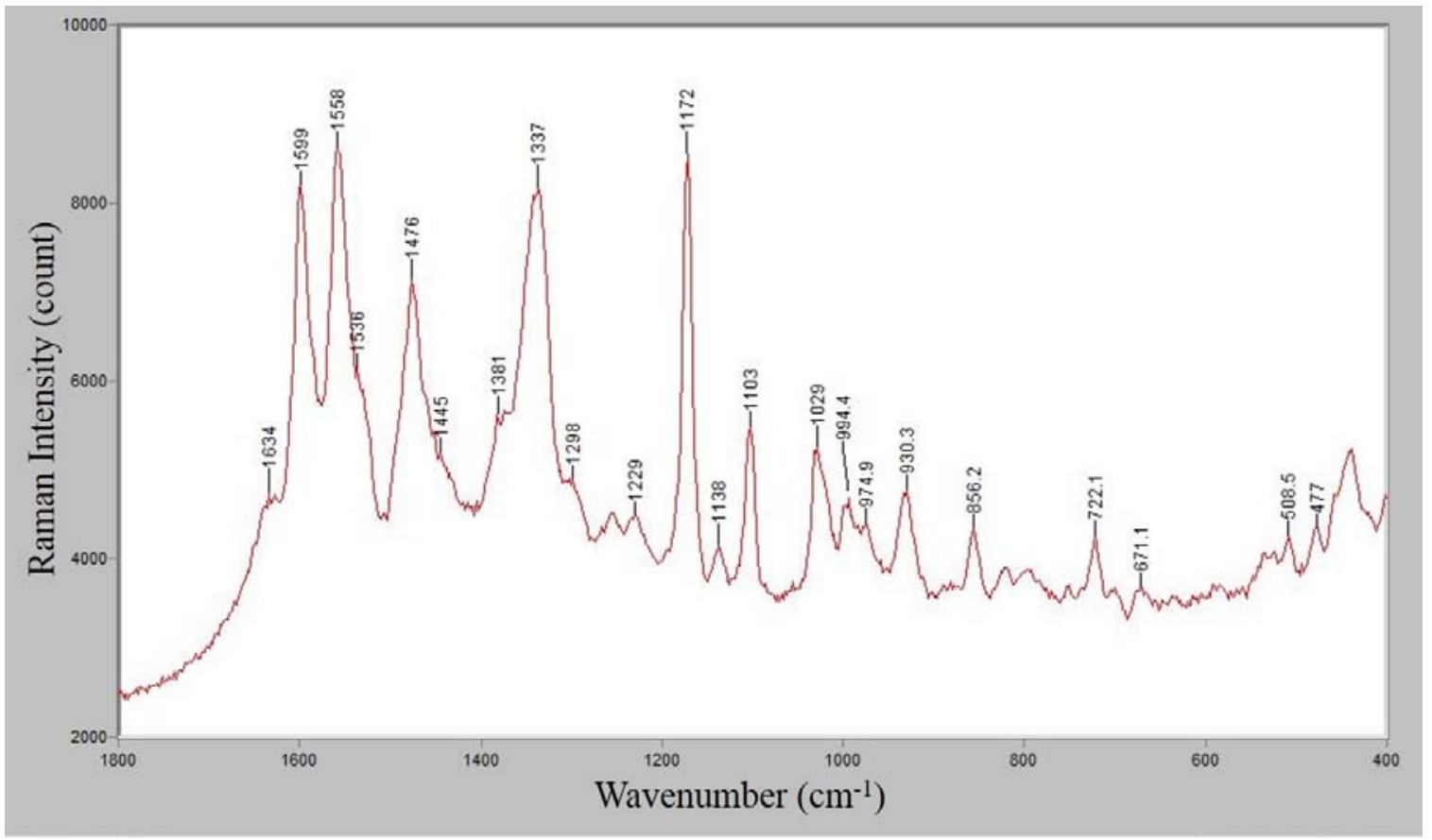

Figure 43: SERS spectra lorazepam

\begin{tabular}{|c|c|c|c|}
\hline Wavenumber & Assignment & Wavenumber & Assignment \\
\hline $1600 \mathrm{~s}$ & Arom $\mathrm{C}=\mathrm{N}$ str (diazepine ring) & $1103 \mathrm{~m}$ & $\begin{array}{l}\text { C-H in plane deformation (a, diazepine } \\
\text { rings) }\end{array}$ \\
\hline $1558 \mathrm{~s}$ & Arom $\mathrm{C}=\mathrm{C}$ str (a-ring) & $1029 \mathrm{~m}$ & Arom C-H str (b-ring) \\
\hline $1476 \mathrm{~s}$ & $\begin{array}{l}\mathrm{C}-\mathrm{H} \text { in plane deformation (a- } \\
\text { ring) }\end{array}$ & $974 \mathrm{w}$ & ----------- \\
\hline 1337 vs & $\begin{array}{l}\mathrm{C}-\mathrm{H} \text { in plane deformation }(\mathrm{b}- \\
\text { ring) }\end{array}$ & $930 \mathrm{w}$ & C-H deformation \\
\hline 1172 vs & C-C-N str (diazepine ring) & $856 \mathrm{w}$ & $\mathrm{C}-\mathrm{C}-\mathrm{N}$ str (diazepine ring) \\
\hline $1137 \mathrm{w}$ & $\begin{array}{l}\mathrm{C}-\mathrm{H} \text { in plane deformation ( } \mathrm{a}, \\
\text { diazepine rings) }\end{array}$ & $671 \mathrm{w}$ & $\mathrm{C}=\mathrm{O}$ deformation \\
\hline
\end{tabular}

Table 21: Observed wavenumbers $\left(\mathrm{cm}^{-1}\right)$ in the surface enhanced Raman spectra of

$$
\text { lorazepam }^{86,61,44}
$$




\subsubsection{Midazolam}

Midazolam is the only 1,2-imidazo-benzodiazpine in this study. It is also the only benzodiazepine with two different halogens on the one molecule, fluorine and chloride.

There are no additional identifying peaks that have not been previously discussed.

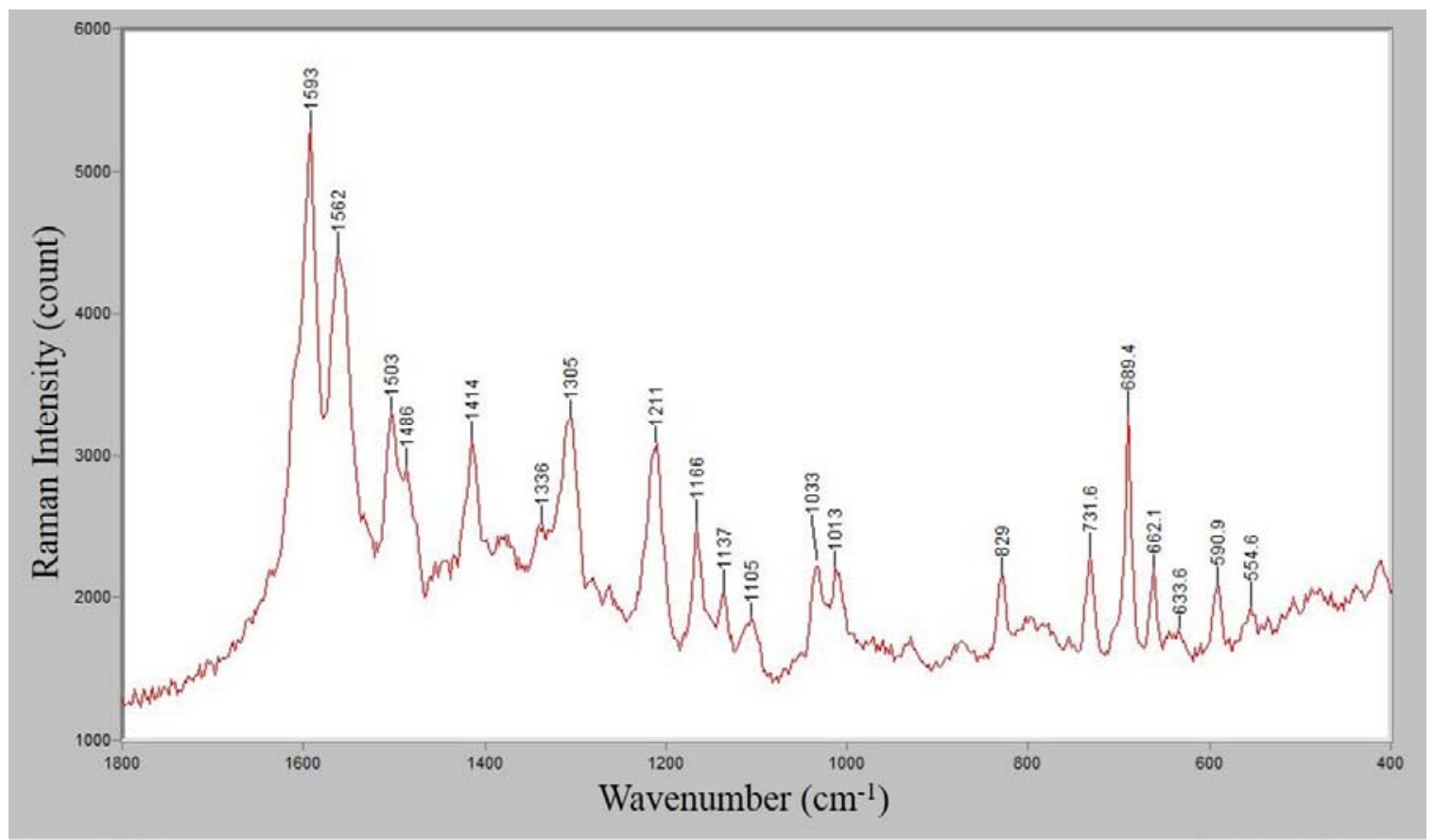

Figure 44: SERS spectra midazolam 


\begin{tabular}{|c|c|c|c|}
\hline Wavenumber & Assignment & Wavenumber & Assignment \\
\hline 1593 vs & Arom $\mathrm{C}=\mathrm{N}$ str (diazepine ring) & $1105 \mathrm{w}$ & $\begin{array}{l}\text { C-H in plane deformation (a, diazepine } \\
\text { rings) }\end{array}$ \\
\hline $1562 \mathrm{~s}$ & Arom $\mathrm{C}=\mathrm{C}$ str (a-ring) & $1033 \mathrm{w}$ & $\mathrm{C}-\mathrm{H}$ in plane deformation (b-ring) \\
\hline $1503 \mathrm{~m}$ & Arom C-H str (b-ring) & $1013 \mathrm{w}$ & Arom C-H str (b-ring) \\
\hline $1485 \mathrm{w}$ sh & Arom $\mathrm{C}=\mathrm{C}$ str (a-ring) & $829 \mathrm{w}$ & $\mathrm{C}-\mathrm{N}-\mathrm{C}$ str (diazepine ring) \\
\hline $1413 \mathrm{~m}$ & Arom $\mathrm{C}=\mathrm{C}$ str (a-ring) & $732 \mathrm{w}$ & ------------------------- \\
\hline $1305 \mathrm{~m}$ & Amide III band (N-ring) & $689 \mathrm{~m}$ & Ring “breathing” (N-ring) \\
\hline $1211 \mathrm{~m}$ & C-F str (b-ring) & $662 \mathrm{w}$ & $\mathrm{C}=\mathrm{C} \operatorname{str}(\mathrm{a}, \mathrm{b}$ rings $)$ \\
\hline $1166 \mathrm{~m}$ & C-C-N str (diazepine ring) & $634 \mathrm{w}$ & ------------------------- \\
\hline $1137 \mathrm{w}$ & Ring "breathing" (N-ring) & $590 \mathrm{w}$ & C-Cl str (a-ring) \\
\hline
\end{tabular}

Table 22: Observed wavenumbers $\left(\mathrm{cm}^{-1}\right)$ in the surface enhanced Raman spectra of

$$
\text { midazolam }^{85}
$$

\subsubsection{Nordiazepam}

Nordiazepam is a 1,4-benzodiazepine that is also the major metabolite of many benzodiazepines in this study. There are no other individual identifying peaks. 


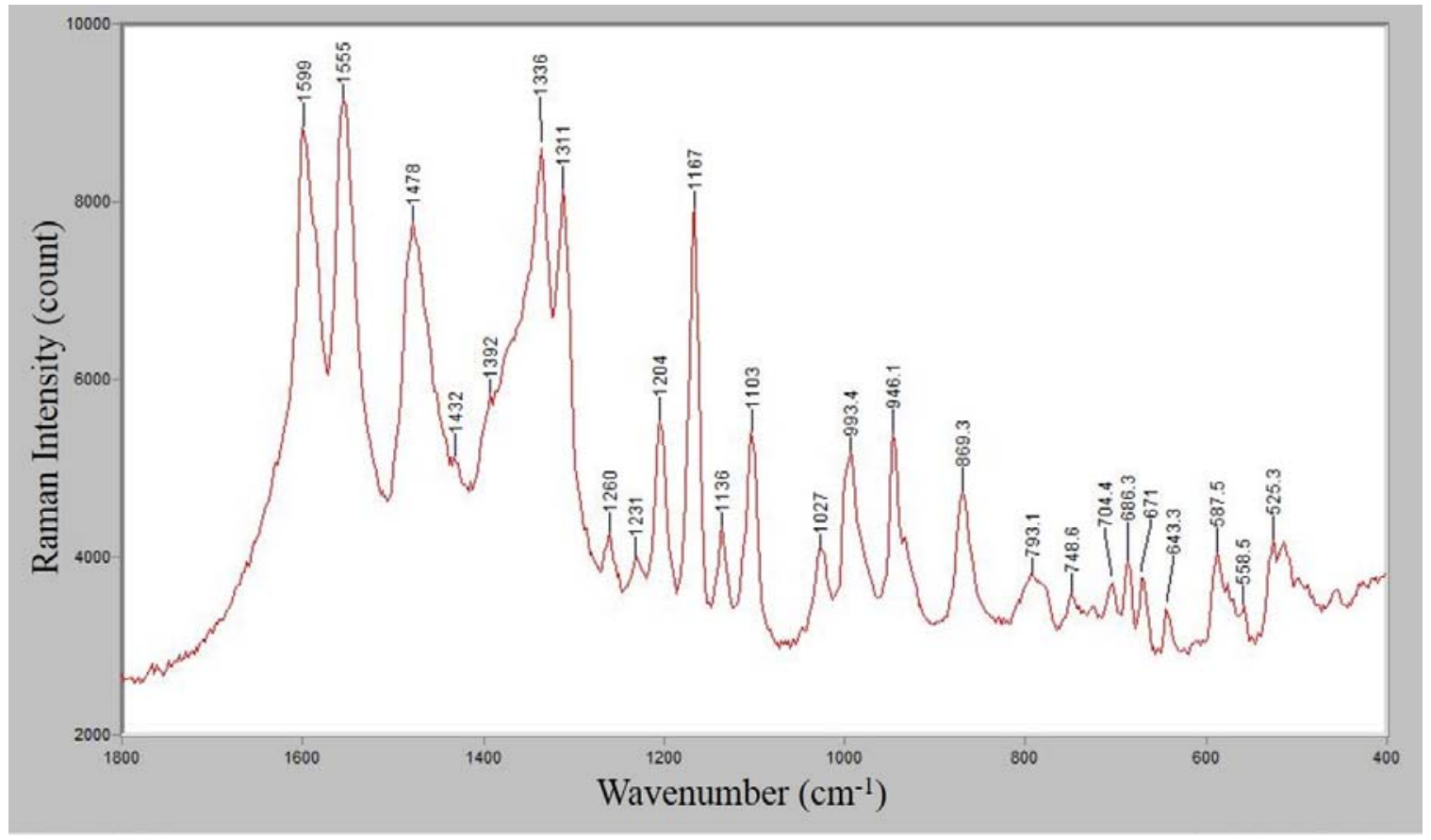

Figure 45: SERS spectra nordiazepam

\begin{tabular}{|c|c|c|c|}
\hline Wavenumber & Assignment & Wavenumber & Assignment \\
\hline 1599 vs & Arom $\mathrm{C}=\mathrm{N}$ str (diazepine ring) & $1027 \mathrm{w}$ & $\mathrm{C}-\mathrm{H}$ in plane deformation (b-ring) \\
\hline $1555 \mathrm{vs}$ & Arom $\mathrm{C}=\mathrm{C}$ str (a-ring) & $993 \mathrm{~m}$ & Monosubstituted "breathing" (b-ring) \\
\hline $1479 \mathrm{~s}$ & $\begin{array}{l}\mathrm{C}-\mathrm{H} \text { in plane deformation (a- } \\
\text { ring) }\end{array}$ & $946 \mathrm{~m}$ & 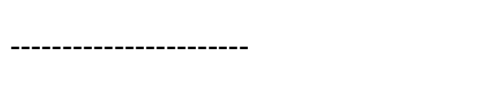 \\
\hline $1336 \mathrm{~s}$ & $\begin{array}{l}\mathrm{C}-\mathrm{H} \text { in plane deformation (a- } \\
\text { ring) }\end{array}$ & $869 \mathrm{~m}$ & C-C-N str (diazepine ring) \\
\hline $1311 \mathrm{~s}$ & $\begin{array}{l}\mathrm{C}-\mathrm{C} \text { in plane deformation (a, } \\
\text { diazepine-rings) }\end{array}$ & $704 \mathrm{w}$ & $\mathrm{C}-\mathrm{H}$ out of plane deformation (b-ring) \\
\hline $1260 \mathrm{w}$ & $\begin{array}{l}\mathrm{C}-\mathrm{C} \text { in plane deformation (b } \\
\text { ring) }\end{array}$ & $686 \mathrm{w}$ & Out of plane ring "breathing" \\
\hline $1230 \mathrm{w}$ & $\mathrm{CH}_{2}$ twist (diazepine ring) & $671 \mathrm{w}$ & $\mathrm{C}=\mathrm{O}$ deformation \\
\hline $1204 \mathrm{~m}$ & $\mathrm{C}_{6} \mathrm{H}_{5}-\mathrm{C}$ vibr. (b-ring) & $643 \mathrm{w}$ & Out of plane deformation (a,b-ring) \\
\hline $1167 \mathrm{~s}$ & C-C-N str (diazepine ring) & $587 \mathrm{w}$ & $\mathrm{C}-\mathrm{Cl}$ str \\
\hline $\begin{array}{l}1136 \mathrm{w} \\
1103 \mathrm{~m}\end{array}$ & $\begin{array}{l}\mathrm{C}-\mathrm{H} \text { in plane deformation (a- } \\
\text { ring) } \\
\mathrm{C}-\mathrm{H} \text { in plane deformation (a, } \\
\text { diazepine rings) }\end{array}$ & $525 \mathrm{w}$ & Out of plane deformation \\
\hline
\end{tabular}

Table 23: Observed wavenumbers $\left(\mathrm{cm}^{-1}\right)$ in the surface enhanced Raman spectra of nordiazepam ${ }^{44,61}$ 


\subsubsection{Oxazepam}

Oxazepam is a 1,4-benzodiazpine and is a major metabolite of many benzodiazepines in this study. There are no additional major identifying peaks.

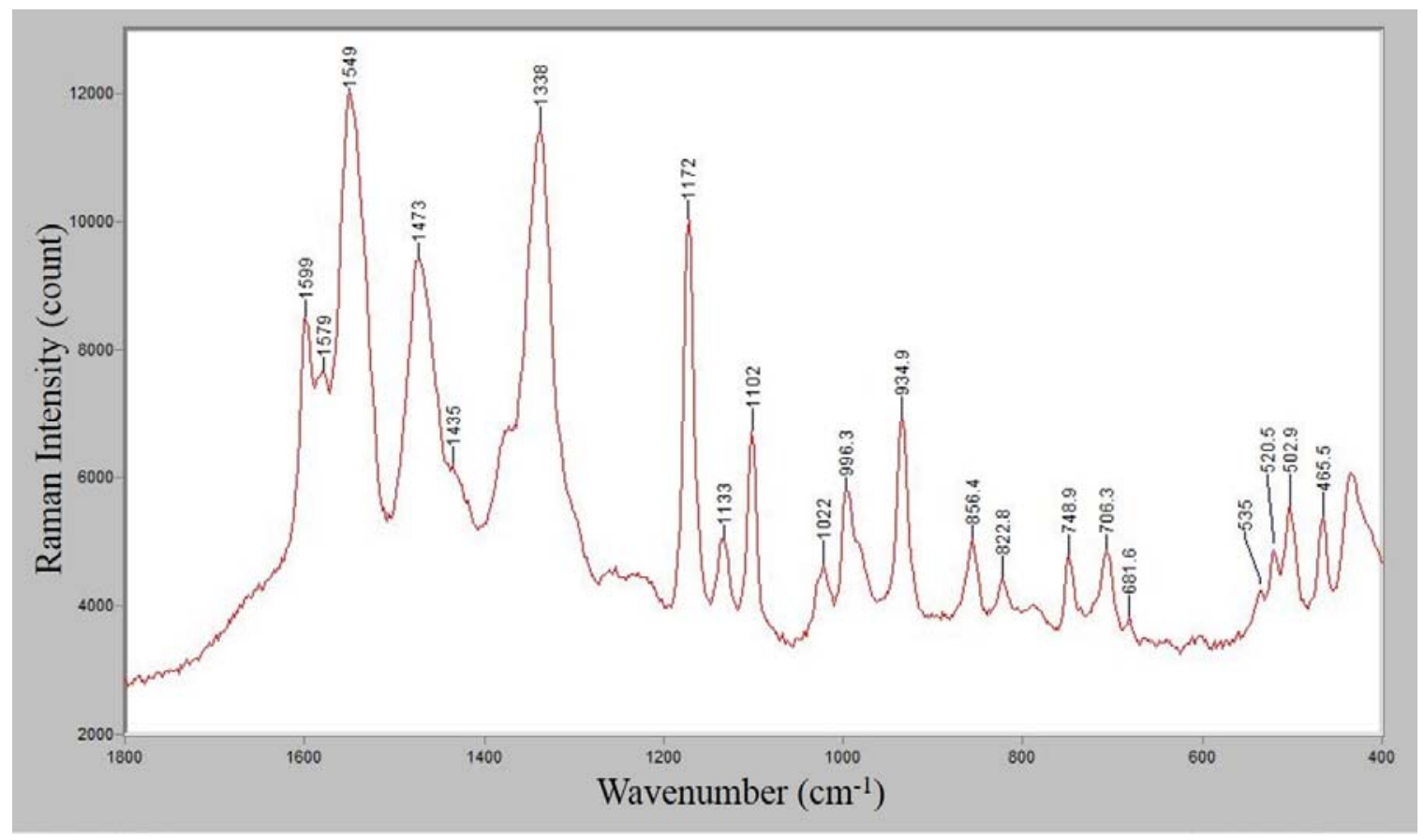

Figure 46: SERS spectra oxazepam

\begin{tabular}{|c|l|c|l|}
\hline Wavenumber & \multicolumn{1}{|c|}{ Assignment } & Wavenumber & \multicolumn{1}{c|}{ Assignment } \\
\hline $1599 \mathrm{~s}$ & Arom C=N str (diazepine ring) & $996 \mathrm{~m}$ & Monosubstituted "breathing" (b-ring) \\
$1473 \mathrm{~s}$ & C-H in plane deformation (a-ring) & $856 \mathrm{w}$ & C-C-N str (diazepine ring) \\
$1337 \mathrm{vs}$ & C-H in plane deformation (a-ring) & $823 \mathrm{w}$ & C-N-C str (diazepine ring) \\
$1172 \mathrm{~s}$ & C-C-N str (diazepine ring) & $749 \mathrm{w}$ & C-H deformation (b-ring) \\
$1133 \mathrm{w}$ & C-H in plane deformation (a-ring) & $706 \mathrm{w}$ & C-H out of plane deformation (b-ring) \\
$1102 \mathrm{~m}$ & ------------------ & $520 \mathrm{w}$ & Out of plane deformation (a,b-ring) \\
$1021 \mathrm{w}$ & C-H in plane deformation (b ring) & & \\
\hline
\end{tabular}

Table 24: Observed wavenumbers $\left(\mathrm{cm}^{-1}\right)$ in the surface enhanced Raman spectra of

$$
\text { oxazepam }^{61,44}
$$




\subsubsection{Triazolam}

Triazolam is a 1,2-triazolo-benzodiazpine. There are no additional major identifying peaks.

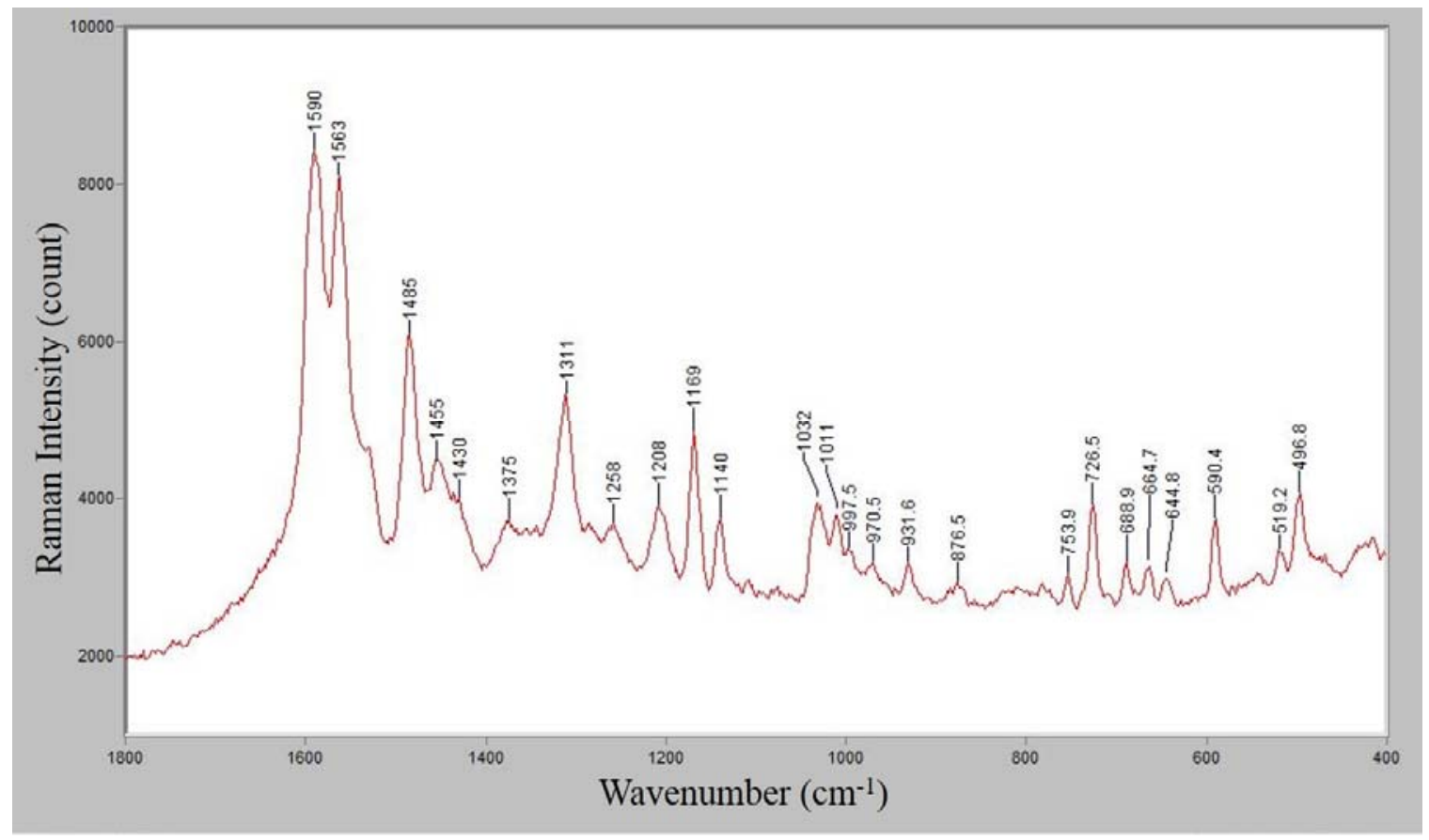

Figure 47: SERS spectra triazolam

\begin{tabular}{|c|l|c|l|}
\hline Wavenumber & \multicolumn{1}{|c|}{ Assignment } & Wavenumber & \multicolumn{1}{c|}{ Assignment } \\
\hline $1590 \mathrm{vs}$ & Arom C=N str (diazepine ring) & $1139 \mathrm{w}$ & Ring "breathing" (N-ring) \\
$1563 \mathrm{vs}$ & Arom C=C str (a-ring) & $1032 \mathrm{w}$ & C-H in plane deformation (b-ring) \\
$1485 \mathrm{~s}$ & Arom C=C str (a-ring) & $1011 \mathrm{w}$ & Trigonal ring "breathing" \\
$1311 \mathrm{~m}$ & C=N-C sym. str (diazepine ring) & $932 \mathrm{w}$ & C-H deformation \\
$1258 \mathrm{w}$ & Ring "breathing" (N-ring) & $727 \mathrm{~m}$ & -------------------- \\
$1208 \mathrm{w}$ & -------------------- & $590 \mathrm{w}$ & C-Cl str (a-ring) \\
$1169 \mathrm{~m}$ & C-C-N str (diazepine ring) & & \\
\hline
\end{tabular}

Table 25: Observed wavenumbers $\left(\mathrm{cm}^{-1}\right)$ in the surface enhanced Raman spectra of triazolam $^{85}$ 


\section{CONCLUSIONS AND FUTURE WORK}

This work shows the development of a specific, sensitive, and rapid screening technique for the detection of trace levels of benzodiazepines in urine. The surface enhanced Raman spectroscopy method was first optimized looking at a number of parameters. Gold nanoparticle that were approximately $30 \mathrm{~nm}$ in size were synthesized using a sodium citrate reduction and were found to provide a higher Raman signal intensity than three types of silver colloidal solutions synthesized through various methods. This was due to the wavelength of laser available for the thesis providing more enhancement for gold than silver at the same wavelength due to the local field intensity enhancement factor. Once the SERS substrate was selected, the concentration and aggregating agent was optimized for the detection of eleven different benzodiazepines. It

was found that when magnesium chloride at a concentration of $16.7 \mathrm{mM}$ was added to the colloidal gold, followed by the addition of drug, the lowest limits of detection ( $0.5-250$ $\mathrm{ng} / \mathrm{mL}$ ) for all eleven benzodiazepines were produced. All the SERS spectra produced were unique to the individual benzodiazepine and were distinguishable.

This sensitive detection technique was then used in conjunction with a fast clean extraction technique, supported liquid extraction. This technique provided efficient extraction of the studied benzodiazepines in fifteen minutes and utilized a sample preparation of dilution of urine with an acetate buffer with a $\mathrm{pH}$ of five. This greatly reduces sample preparation time when compared to other common sample preparation methods such as enzymatic hydrolysis combined with solid phase extraction. Limits of detection of 6-640 $\mathrm{ng} / \mathrm{mL}$ in urine were determined with the optimized SERS detection. This extraction technique was also found to be specific enough to effectively extract the 
benzodiazepines without coextracting common interference compounds that also produced high Raman signals using the optimized SERS method and are found in drug facilitated sexual assault victims. Those benzodiazepines with high limits of detection were the tricyclic benzodiazepines. It can be speculated that the additional ring could be contributing to the inefficient extraction by interacting with another compound in the urine or inefficiently eluting from the supported liquid extraction sorbent. These drugs also form glucuronides in urine through metabolism and may be reducing the extraction efficiency due to the lack of enzymatic hydrolysis in the optimized supported liquid extraction. In order to improve this work, other extraction techniques, that can still reduce the time necessary for other current techniques, need to be utilized in order to improve the detection limits of the tricyclic benzodiazepines used in this study such as alprazolam. Future work should also examine the cause of this change in efficiency between the extraction of the 1,4-benzodiazpines and the tricyclic classes of benzodiazepines from urine.

In addition, through the interference studies it was found that $\Delta^{9}$ tetrahydrocannabinol (THC) and codeine provided high intensity SERS spectra using the optimized method designed for benzodiazepines. Future work could be adapted from the current method for the sensitive detection of THC and synthetic cannabinoids as well as other opiates. Synthetic cannabinoids are an increasing problem in the forensics community and a sensitive detection method that could provide information about their identity would prove useful as their structures are constantly being changed creating more compounds. This type of method would allow for the rapid screening for these drugs in a 
clinical setting where a doctor may not know what is causing a patient's reaction and current immunoassays do not detect for all the synthetic cannabinoids.

This method could also be applied to a microfluidic system in the future in order to decrease the amount of sample necessary for analysis as well as the time required. Microfluidic systems also can be designed to have the advantage of providing sample preparation and analysis in one complete system. The extraction column could be embedded in the channel of the microfluidic chip and due to benefit of waste being retained by the cartridge and only the analytes of interest being eluted, the analyte could then be detected further down the channel without the need for removing the waste. A mixing channel would provide a place to combine the gold nanoparticles, aggregating agent, and analyte and detection would be performed through the chip as long as the glass used does not produce interferences.

Overall this combined supported liquid extraction with surface-enhanced Raman spectroscopy method has demonstrated a sensitive and selective extraction and detection, without interference from common contaminants all in a time of less than twenty minutes. This lowers the time required to process a sample for screening in a drug facilitated sexual assault case. This method also provides spectral information about the individual benzodiazepine present though Raman specific peaks allowing preliminary identification of the drug though a screening test. 


\section{LIST OF REFERENCES}

1. Sternbach, L. H., The benzodiazepine story. Journal of medicinal chemistry 1979, $22(1), 1-7$.

2. Sternbach, L. H., I II II. Google Patents: 1959.

3. Brenneisen, R.; Raymond, L., Pharmacology of flunitrazepam and other benzodiazepines. Humana Press: NJ: 2001; pp 1-15.

4. INCB, Psycotropic Substances Report 2012: Statistics for 2011 Assesment of annual Medical and Scientific Requirements for Substances in Schedules II, III, and IV of the Convention on Psychotropic Substances of 1971. Board, I. N. C., Ed. United Nations: New York 2012.

5. Levine, B., Principles of forensic toxicology. Amer. Assoc. for Clinical Chemistry: 2003.

6. Doctor, E. L.; McCord, B., Comparison of aggregating agents for the surfaceenhanced Raman analysis of benzodiazepines. Analyst 2013, 138 (20), 59265932 .

7. Breimer, D., Pharmacokinetics and metabolism of various benzodiazepines used as hypnotics. British journal of clinical pharmacology 1979, 8 (Supplement s1), 7S-13S.

8. LeBeau, M. A., Drug-Facilitated Sexual Assault. Wiley Online Library: 2001.

9. Juhascik, M.; Le, N. L.; Tomlinson, K.; Moore, C.; Gaensslen, R.; Negrusz, A., Development of an analytical approach to the specimens collected from victims of sexual assault. Journal of analytical toxicology 2004, 28 (6), 400406.

10. LeBeau, M.; Andollo, W.; Hearn, W. L.; Baselt, R.; Cone, E.; Finkle, B.; Fraser, D.; Jenkins, A.; Mayer, J.; Negrusz, A., Recommendations for toxicological investigations of drug-facilitated sexual assaults. Journal of forensic sciences 1999, 44 (1), 227.

11. LeBeau, M. A., Guidance for improved detection of drugs used to facilitate crimes. Therapeutic drug monitoring 2008, 30 (2), 229-233.

12. Elsohly, M. A.; Salamone, S., Prevalence of drugs used in cases of alleged sexual assault. Journal of analytical toxicology 1999, 23 (3), 141-146. 
13. Salamone, S.; Honasoge, S.; Brenner, C.; McNally, A.; Passarelli, J.; GocSzkutnicka, K.; Brenneisen, R.; ElSohly, M.; Feng, S., Flunitrazepam excretion patterns using the Abuscreen OnTrak and OnLine immunoassays: comparison with GC-MS. Journal of analytical toxicology 1997, 21 (5), 341345 .

14. Salamone, S. J., Benzodiazepines and GHB: Detection and pharmacology. Humana Pr Inc: 2001.

15. Tenore, P. L., Advanced urine toxicology testing. Journal of addictive diseases 2010, 29 (4), 436-448.

16. Forsman, M.; Nyström, I.; Roman, M.; Berglund, L.; Ahlner, J.; Kronstrand, R., Urinary detection times and excretion patterns of flunitrazepam and its metabolites after a single oral dose. Journal of analytical toxicology 2009, 33 (8), 491-501.

17. Kintz, P.; Villain, M.; Cirimele, V.; Pépin, G.; Ludes, B., Windows of detection of lorazepam in urine, oral fluid and hair, with a special focus on drug-facilitated crimes. Forensic science international 2004, 145 (2), 131135.

18. Smink, B.; Mathijssen, M.; Lusthof, K.; De Gier, J.; Egberts, A.; Uges, D., Comparison of urine and oral fluid as matrices for screening of thirty-three benzodiazepines and benzodiazepine-like substances using immunoassay and LC-MS (-MS). Journal of analytical toxicology 2006, 30 (7), 478-485.

19. Augsburger, M.; Rivier, L.; Mangin, P., Comparison of different immunoassays and GC-MS screening of benzodiazepines in urine. Journal of pharmaceutical and biomedical analysis 1998, 18 (4-5), 681-687.

20. Lu, N. T.; Taylor, B. G., Drug screening and confirmation by GC-MS: Comparison of EMIT II and Online KIMS against 10 drugs between US and England laboratories. Forensic science international 2006, 157 (2-3), 106116.

21. Schütz, H., Modern screening strategies in analytical toxicology with special regard to new benzodiazepines. Zeitschrift für Rechtsmedizin 1988, 100 (1), $19-37$.

22. Meatherall, R.; Fraser, A. D., Comparison of four immunoassays for the detection of lorazepam in urine. Therapeutic drug monitoring 1998, 20 (6), 673-675. 
23. Papoutsis, I. I.; Athanaselis, S. A.; Nikolaou, P. D.; Pistos, C. M.; Spiliopoulou, C. A.; Maravelias, C. P., Development and validation of an EIGC-MS method for the determination of benzodiazepine drugs and their metabolites in blood: Applications in clinical and forensic toxicology. Journal of pharmaceutical and biomedical analysis 2010, 52 (4), 609-614.

24. Raman, C.; Krishnan, K., A new type of secondary radiation. Nature 1928, 121 (3048), 501-502.

25. Le Ru, E.; Etchegoin, P., Principles of surface-enhanced Raman spectroscopy: and related plasmonic effects. Access Online via Elsevier: 2008.

26. Skoog, D. A.; Holler, F. J.; Nieman, T. A., Principles of instrumental analysis. 1998.

27. Walrafen, G. E.; Hokmabadi, M.; Yang, W.; Chu, Y.; Monosmith, B., Collision-induced Raman scattering from water and aqueous solutions. Journal of physical chemistry 1989, 93 (8), 2909-2917.

28. Fleischmann, M.; Hendra, P.; McQuillan, A., Raman spectra of pyridine adsorbed at a silver electrode. Chemical physics letters 1974, 26 (2), 163-166.

29. Jeanmaire, D. L.; Van Duyne, R. P., Surface Raman spectroelectrochemistry: Part I. Heterocyclic, aromatic, and aliphatic amines adsorbed on the anodized silver electrode. Journal of electroanalytical chemistry and interfacial electrochemistry 1977, 84 (1), 1-20.

30. Albrecht, M. G.; Creighton, J. A., Anomalously intense Raman spectra of pyridine at a silver electrode. Journal of the American chemical society 1977, 99 (15), 5215-5217.

31. Kiefer, W.; Schlücker, S., Surface enhanced Raman spectroscopy: analytical, biophysical and life science applications. John Wiley \& Sons: 2013.

32. Born, M.; Wolf, E., Principles of optics: electromagnetic theory of propagation, interference and diffraction of light. CUP Archive: 1999.

33. Griffiths, D. J.; College, R., Introduction to electrodynamics. Prentice hall Upper Saddle River, NJ: 1999; Vol. 3.

34. Rojas, R.; Claro, F., Theory of surface enhanced Raman scattering in colloids. The Journal of chemical physics 1993, 98 (2), 998-1006. 
35. Campion, A.; Kambhampati, P., Surface-enhanced Raman scattering. Chemical society reviews 1998, 27 (4), 241-250.

36. Cyrankiewicz, M.; Wybranowski, T.; Kruszewski, S. In Study of SERS efficiency of metallic colloidal systems, Journal of Physics: Conference Series, IOP Publishing: 2007; p 012013.

37. Yaffe, N. R.; Ingram, A.; Graham, D.; Blanch, E. W., A multi-component optimisation of experimental parameters for maximising SERS enhancements. Journal of Raman spectroscopy 2010, 41 (6), 618-623.

38. Abdali, S.; Johannessen, C.; Nygaard, J.; Nørbygaard, T., Resonance surface enhanced Raman optical activity of myoglobin as a result of optimized resonance surface enhanced Raman scattering conditions. Journal of physics: condensed matter 2007, 19 (28), 285205.

39. Lee, P. C.; Meisel, D., Adsorption and surface-enhanced Raman of dyes on silver and gold sols. Journal of physical chemistry 1982, 86 (17), 3391-3395.

40. Cinta, S.; Iliescu, T.; Astilean, S.; David, L.; Cozar, O.; Kiefer, W., 1,4benzodiazepine drugs adsorption on the Ag colloidal surface. Journal of molecular structure 1999, 482, 685-688.

41. Rana, V.; Canamares, M. V.; Kubic, T.; Leona, M.; Lombardi, J. R., Surfaceenhanced Raman spectroscopy for trace identification of controlled Substances: Morphine, Codeine, and Hydrocodone. Journal of forensic sciences 2011, 56 (1), 200-207.

42. Fox, J. D.; Waverka, K. N.; Verbeck, G. F., Gold-plating of Mylar lift films to capitalize on surface enhanced Raman spectroscopy for chemical extraction of drug residues. Forensic science international 2012, 216 (1-3), 141-145.

43. Sagmuller, B.; Schwarze, B.; Brehm, G.; Trachta, G.; Schneider, S., Identification of illicit drugs by a combination of liquid chromatography and surface-enhanced Raman scattering spectroscopy. Journal of molecular structure 2003, 661, 279-290.

44. Trachta, G.; Schwarze, B.; Brehm, G.; Schneider, S.; Hennemann, M.; Clark, T., Near-infrared Fourier transform surface-enhanced Raman scattering spectroscopy of 1,4-benzodiazepine drugs employing gold films over nanospheres. Journal of Raman spectroscopy 2004, 35 (5), 368-383.

45. Trachta, G.; Schwarze, B.; Sagmuller, B.; Brehm, G.; Schneider, S., Combination of high-performance liquid chromatography and SERS detection 
applied to the analysis of drugs in human blood and urine. Journal of molecular structure 2004, 693 (1-3), 175-185.

46. Sutherland, W. S.; Laserna, J. J.; Angebranndt, M. J.; Winefordner, J. D., Surface-enhanced Raman analysis of sulfa drugs on colloidal silver dispersion. Analytical chemistry 1990, 62 (7), 689-693.

47. Sagmuller, B.; Schwarze, B.; Brehm, G.; Schneider, S., Application of SERS spectroscopy to the identification of (3,4-methylenedioxy) amphetamine in forensic samples utilizing matrix stabilized silver halides. Analyst 2001, 126 (11), 2066-2071.

48. Bell, S. E. J.; Barrett, L. J.; Burns, D. T.; Dennis, A. C.; Speers, S. J., Tracking the distribution of "ecstasy" tablets by Raman composition profiling: A large scale feasibility study. Analyst 2003, 128 (11), 1331-1335.

49. Bell, S. E. J.; Beattie, J. R.; McGarvey, J. J.; Peters, K. L.; Sirimuthu, N. M. S.; Speers, S. J., Development of sampling methods for Raman analysis of solid dosage forms of therapeutic and illicit drugs. Journal of Raman spectroscopy 2004, 35 (5), 409-417.

50. Bell, S. E. J.; Burns, D. T.; Dennis, A. C.; Matchett, L. J.; Speers, J. S., Composition profiling of seized ecstasy tablets by Raman spectroscopy. Analyst 2000, 125 (10), 1811-1815.

51. Bell, S. E. J.; Burns, D. T.; Dennis, A. C.; Speers, J. S., Rapid analysis of ecstasy and related phenethylamines in seized tablets by Raman spectroscopy. Analyst 2000, 125 (3), 541-544.

52. Bell, S. E. J.; Fido, L. A.; Sirimuthu, N. M. S.; Speers, S. J.; Peters, K. L.; Cosbey, S. H., Screening tablets for DOB using surface-enhanced Raman spectroscopy. Journal of forensic sciences 2007, 52 (5), 1063-1067.

53. Ryder, A. G., Surface enhanced Raman scattering for narcotic detection and applications to chemical biology. Current opinion in chemical biology 2005, 9 (5), 489-493.

54. Dijkstra, R. J.; Gerssen, A.; Efremov, E. V.; Ariese, F.; Brinkman, U. A. T.; Gooijer, C., Substrates for the at-line coupling of capillary electrophoresis and surface-enhanced Raman spectroscopy. Analytica chimica Acta 2004, 508 (2), $127-134$.

55. Inscore, F.; Shende, C.; Sengupta, A.; Huang, H.; Farquharson, S., Detection of Drugs of Abuse in Saliva by Surface-Enhanced Raman Spectroscopy (SERS). Applied spectroscopy 2011, 65 (9), 1004-1008. 
56. Leopold, N.; Lendl, B., A new method for fast preparation of highly surfaceenhanced Raman scattering (SERS) active silver colloids at room temperature by reduction of silver nitrate with hydroxylamine hydrochloride. Journal of physical chemistry B 2003, 107 (24), 5723-5727.

57. Creighton, J. A.; Blatchford, C. G.; Albrecht, M. G., Plasma resonance enhancement of Raman scattering by pyridine adsorbed on silver or gold sol particles of size comparable to the excitation wavelength. Journal of the chemical society, Faraday transactions 2 1979, 75, 790-798.

58. Fini, G., Applications of Raman spectroscopy to pharmacy. Journal of Raman spectroscopy 2004, 35 (5), 335-337.

59. Vankeirsbilck, T.; Vercauteren, A.; Baeyens, W.; Van der Weken, G.; Verpoort, F.; Vergote, G.; Remon, J. P., Applications of Raman spectroscopy in pharmaceutical analysis. Trends in analytical chemistry 2002, 21 (12), 869877.

60. Neville, G. A.; Beckstead, H. D.; Shurvell, H. F., A Fourier-transform Raman and infrared vibrational study of delorazepam, fludiazepam, flurazepam, and tetrazepam. Journal of pharmaceutical sciences 1994, 83 (2), 143-151.

61. Neville, G. A.; Shurvell, H. F., Fourier-transform Raman and infrared vibrational study of diazepam and 4 closely realated 1,4-benzodiazpeines. Journal of Raman spectroscopy 1990, 21 (1), 9-19.

62. Neville, G. A.; Beckstead, H. D.; Shurvell, H. F., A Fourier-transform Raman and IR vibrational study of flurazepam base and the monohydrochloride and dihydrochloride salts. Journal of pharmaceutical sciences 1995, 84 (2), 179184.

63. Svec, F., Less common applications of monoliths: Preconcentration and solidphase extraction. Journal of chromatography B 2006, 841 (1-2), 52-64.

64. Mosier-Boss, P. A.; Lieberman, S. H., Surface-enhanced Raman spectroscopy substrate composed of chemically modified gold colloid particles immobilized on magnetic microparticles. Analytical chemistry 2005, 77 (4), 1031-1037.

65. Louter, A.; Bosma, E.; Schipperen, J.; Vreuls, J.; Brinkman, U., Automated on-line solid-phase extraction-gas chromatography with nitrogen-phosphorus detection: determination of benzodiazepines in human plasma. Journal of chromatography B 1997, 689 (1), 35-43. 
66. Akerman, K.; Jolkkonen, J.; Parviainen, M. a.; Penttilä, I., Analysis of lowdose benzodiazepines by HPLC with automated solid-phase extraction. Clinical chemistry 1996, 42 (9), 1412-1416.।

67. Inoue, H.; Maeno, Y.; Iwasa, M.; Matoba, R.; Nagao, M., Screening and determination of benzodiazepines in whole blood using solid-phase extraction and gas chromatography/mass spectrometry. Forensic science international 2000, 113 (1), 367-373.

68. Hegstad, S.; Øiestad, E.; Johansen, U.; Christophersen, A., Determination of benzodiazepines in human urine using solid-phase extraction and highperformance liquid chromatography-electrospray ionization tandem mass spectrometry. Journal of analytical toxicology 2006, 30 (1), 31-37.

69. Ghobadi, M.; Yamini, Y.; Ebrahimpour, B., SPE coupled with dispersive liquid-liquid microextraction followed by GC with flame ionization detection for the determination of ultra-trace amounts of benzodiazepines. Journal of separation science 2014, 37 (3), 287-294.

70. Bishop, S. C.; Lerch, M.; McCord, B. R., Micellar electrokinetic chromatographic screening method for common sexual assault drugs administered in beverages. Forensic science international 2004, 141 (1), 7-15.

71. Bishop, S. C.; Lerch, M.; McCord, B. R., Detection of nitrated benzodiazepines by indirect laser-induced fluorescence detection on a microfluidic device. Journal of chromatography A 2007, 1154 (1), 481-484.

72. Blas, M.; McCord, B. R., Determination of trace levels of benzodiazepine in urine using capillary electrochromatography-time of flight mass spectrometry. Electrophoresis 2008, 29 (10), 2182-2192.

73. Biotage, Extraction of Benzodiazepines From Human Urine Using ISOLUTE® SLE+ 96-Well Plates and Columns Prior to LC-MS/MS Analysis. Biotage: 2011.

74. Trocewicz, J., Urine sample preparation of tricyclic antidepressants by means of a supported liquid membrane technique for high-performance liquid chromatographic analysis. Journal of chromatography B 2004, 801 (2), 213220 .

75. Sauve, E.; Langødegård, M.; Ekeberg, D.; Øiestad, Å., Determination of benzodiazepines in ante-mortem and post-mortem whole blood by solidsupported liquid-liquid extraction and UPLC-MS/MS. Journal of chromatography B 2012, 883, 177-188. 
76. Van der Merwe, P.; Müller, F.; Müller, F., Caffeine in sport. Urinary excretion of caffeine in healthy volunteers after intake of common caffeine-containing beverages. South African medical journal= Suid-Afrikaanse tydskrif vir geneeskunde 1988, 74 (4), 163-164.

77. Haller, C.; Thai, D.; Jacob, P.; Dyer, J. E., GHB urine concentrations after single-dose administration in humans. Journal of analytical toxicology 2006, 30 (6), 360-364.

78. Oyler, J. M.; Cone, E. J.; Joseph, R. E.; Huestis, M. A., Identification of hydrocodone in human urine following controlled codeine administration. Journal of analytical toxicology 2000, 24 (7), 530-535.

79. De Cremer, K.; Van Overmeire, I.; Van Loco, J., On-line solid-phase extraction with ultra performance liquid chromatography and tandem mass spectrometry for the detection of nicotine, cotinine and trans-3'hydroxycotinine in urine to strengthen human biomonitoring and smoking cessation studies. Journal of pharmaceutical and biomedical analysis 2013, $76,126-133$.

80. Smith, M. L.; Shimomura, E.; Paul, B. D.; Cone, E. J.; Darwin, W. D.; Huestis, M. A., Urinary excretion of ecgonine and five other cocaine metabolites following controlled oral, intravenous, intranasal, and smoked administration of cocaine. Journal of analytical toxicology 2010, 34 (2), 5763.

81. Lowe, R. H.; Abraham, T. T.; Darwin, W. D.; Herning, R.; Cadet, J. L.; Huestis, M. A., Extended urinary $\Delta 9$-tetrahydrocannabinol excretion in chronic cannabis users precludes use as a biomarker of new drug exposure. Drug and alcohol dependence 2009, 105 (1), 24-32.

82. Jafari, M.; Badihi, Z.; Jazan, E., A new approach to determine salicylic acid in human urine and blood plasma based on negative electrospray ion mobility spectrometry after selective separation using a molecular imprinted polymer. Talanta 2012, 99, 520-526.

83. Bose, D.; Durgbanshi, A.; Martinavarro-Dominguez, A.; Capella-Peiró, M.; Carda-Broch, S.; Esteve-Romero, J.; Gil-Agusti, M., Rapid determination of acetaminophen in physiological fluids by liquid chromatography using SDS mobile phase and ED detection. Journal of chromatographic science 2005, 43 (6), 313-318.

84. Jung, J. H.; Choo, J.; Kim, D. J.; Lee, S., Quantitative determination of nicotine in a PDMS microfluidic channel using surface enhanced Raman spectroscopy. Bulletin of the Korean Chemical Society 2006, 27 (2), 277-280. 
85. Asselin, E. L. A Raman and infrared vibrational study of 1,2-triazolobenzodiazepines. Cedar Crest College, Allentown, PA, 2008.

86. Lin-Vien, D.; Colthup, N. B.; Fateley, W. G.; Grasselli, J. G., The handbook of infrared and Raman characteristic frequencies of organic molecules. Elsevier: 1991.

87. Sigel, Erwin. Mapping of the benzodiazepine recognition site on GABA-A receptors. Current topics in medicinal chemistry 2002, 2 (8), 833-839. 
APPENDICES 
APPENDIX A: Use of Raman instrument Perkin Elmer Raman Station 400F using Spectrum v.6.35 software

1. Turn on in order
a. Computer
b. Raman station $400 \mathrm{~F}$ with the laser

2. Open Spectrum software
a. User name Administrator $->\mathrm{OK}$
b. Select Instrument (RamanStation 400)

3. Instrumentation settings/specifications are starts automatically (do not halt)
a. Resetting beam path motor
b. Stabilization of laser (60 seconds)
c. Temperature setting $(-50 \mathrm{C})$

4. Once instrument is cooled and stabilized software opens automatically to an empty spectrum. Make sure the sample holder is not in the sampling compartment

5. Select Setup -> Instrument -> Instrument (Figure 48)

a. Laser Power: $100 \%$ (gives the best spectral views)

i. Power is $350 \mathrm{~mW}$ or $100 \mathrm{~mW}$ at sample surface with this setting

b. Beam path: Sample Compartment

c. Sample holder: Versatile- Cuvette 


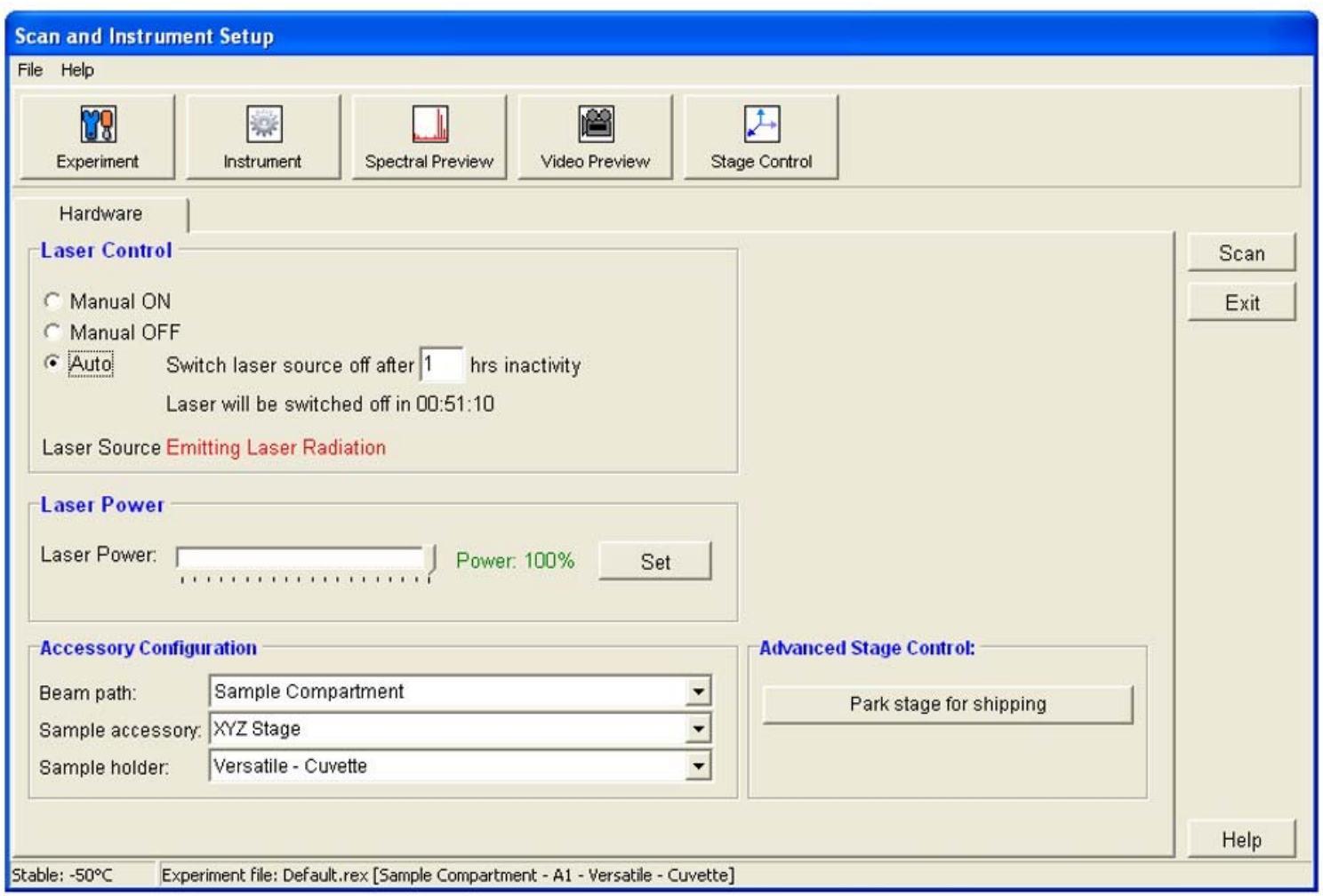

Figure 48: Instrument setup screen shot

d. Confirm these accessory configurations. If the sample holder on the stage is different to that selected on the accessory configuration in the instrument setting dialog, it may cause severe damage to the instrument (jamming the motorized stage and changing the alignment of the stage when sample holder is switched. The Lens Snout (Figure 49) should be in the correct orientation for the sample. 


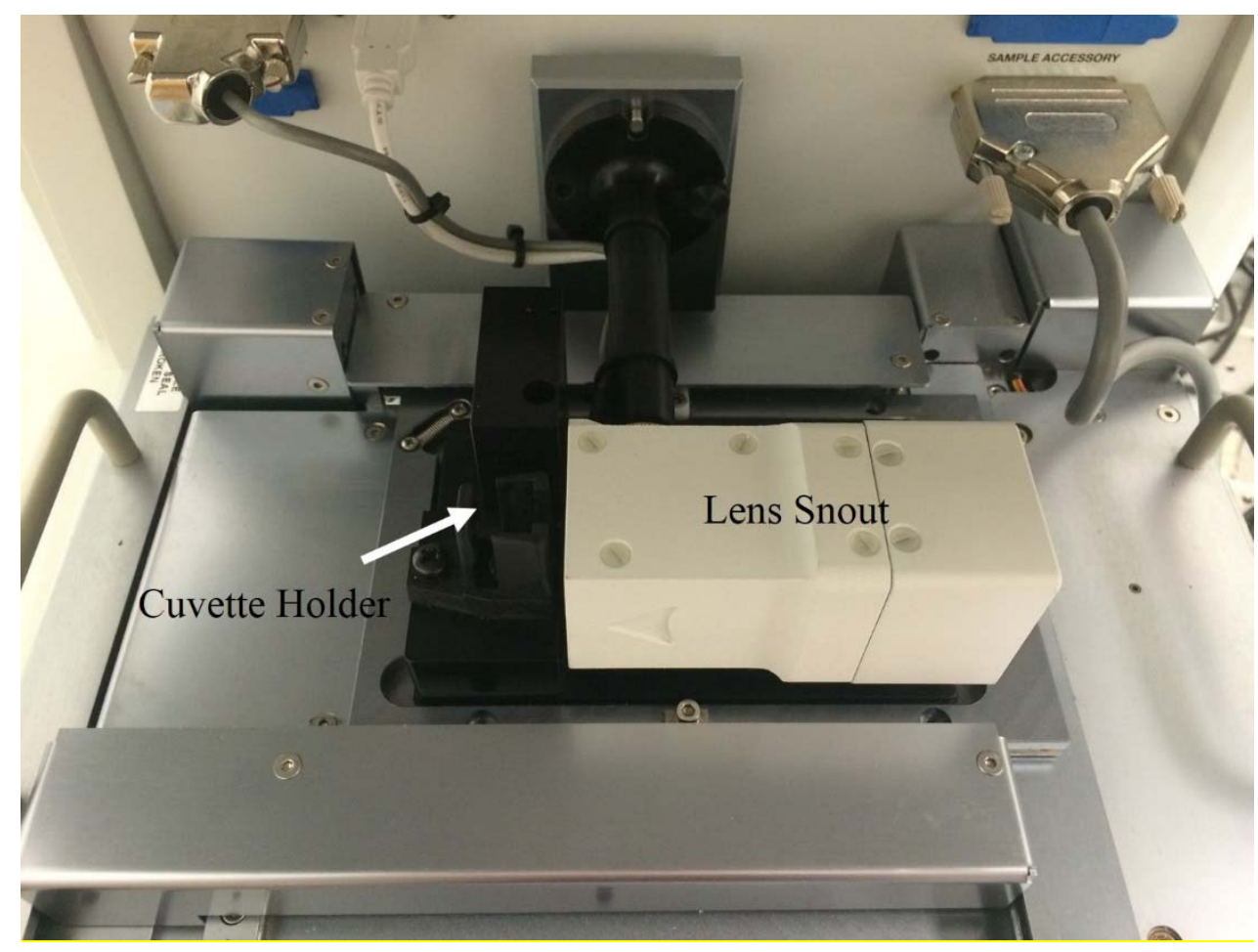

Figure 49: Picture of proper configuration with sample holder for cuvette analysis

6. Select Setup -> Instrument -> Experiment

a. Setup (Figure 50)

i. Set exposure - Keep exposure time between $1-5$ seconds. 10 seconds is the maximum exposure however it may lead to saturation of the detector

ii. Set number of exposures -4 


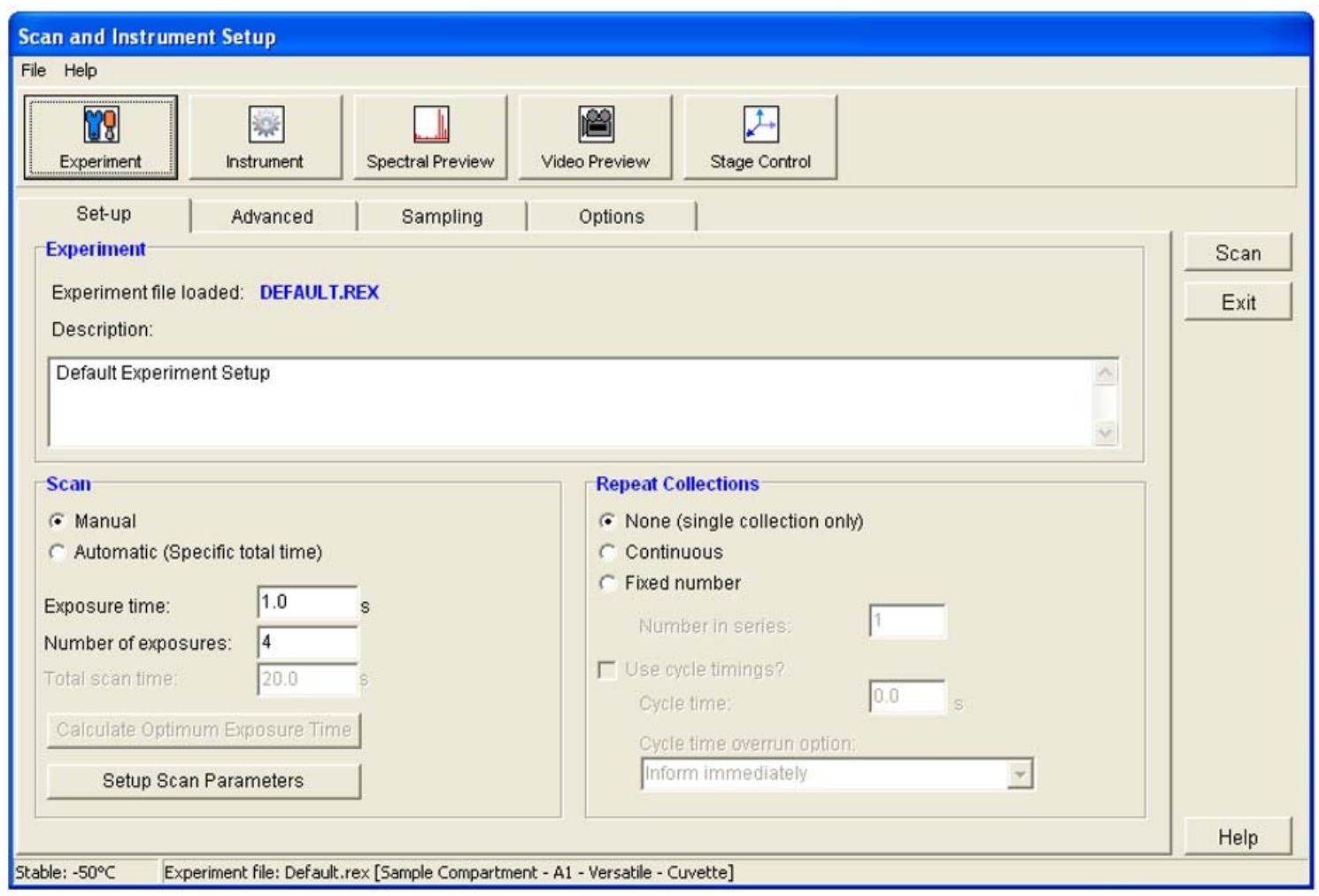

Figure 50: Experiment setup screen shot

b. Advanced (Figure 51)

i. Spectral information allows for selection of wavenumber range. It is recommended to scan the full range $(200-3278 \mathrm{~cm}-1)$

ii. Background handling - Deselect $\#$ Backgrounds $=\#$ Scans

iii. Set specific number of background scans to 2 


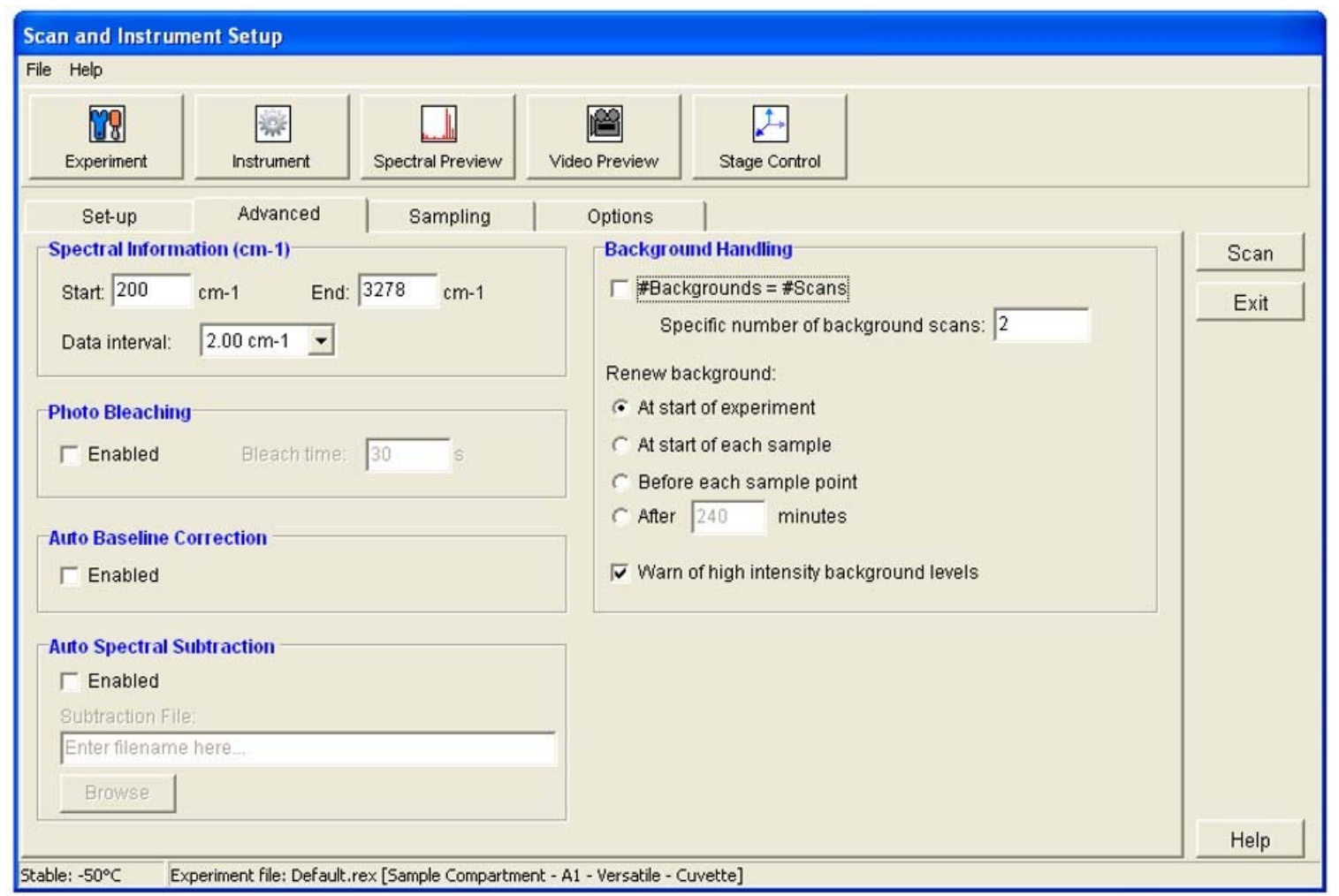

Figure 51: Advanced experimental setup screen shot

7. Select Instrument $->$ Scan Figure 52

a. Type out Sample Name Prefix

b. Select "Archive collected data" and assign location

i. This will automatically save the scan to the location selected but will not automatically open in Spectrum

ii. If "Send collected data to Spectrum" is selected, the spectrum will automatically open following scan however it will not automatically save. 


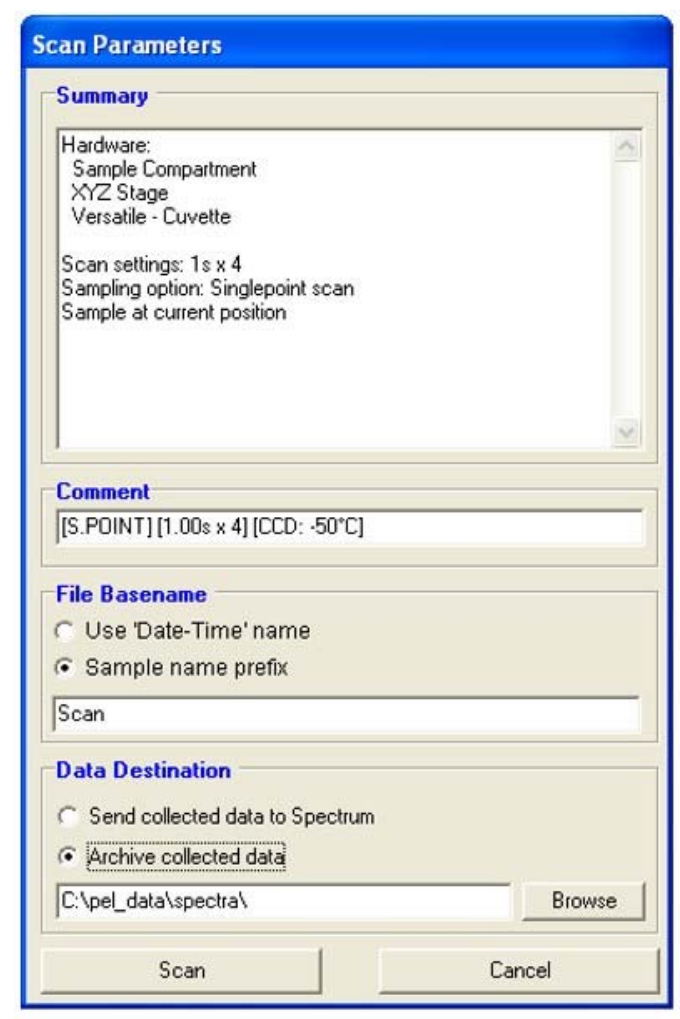

Figure 52: Scan data collection screen shot

8. All scans are automatically saves as *.SP files which can be opened in any version of Spectrum software. Other useful file names:
a. *.SPC - editing files using PE GRAMS software
b. *.CHR - editing files using Excel software

9. Instrument should be shut down in the following order
a. Close Spectrum software
b. Turn off Raman Station 400F/Laser
c. Shut down computer 
APPENDIX B: Standard operating procedure for gold nanoparticle synthesis ${ }^{39,36}$

1. Prepare $10 \mathrm{~mL}$ of $1 \%$ by weight trisodium citrate in water.

2. Prepare $100 \mathrm{~mL}$ of a $0.5 \mathrm{mM} \mathrm{HAuCl}_{4}$ in water. Heat solution to boiling under reflux with vigorous stirring.

3. Add $4 \mathrm{~mL}$ of $1 \%$ tridsodium citrate solution to the boiling $0.5 \mathrm{mM} \mathrm{HAuCl}_{4}$ solution.

4. Continue boiling and stirring for 30 minutes.

5. Remove from heat and allow to cool to room temperature.

6. Dilute solution to $100 \mathrm{~mL}$ following cooling.

7. Resulting solution should be clear and dark red in color with a UV absorption maximum at $522 \mathrm{~nm}$. 
APPENDIX C: Standard operating procedure for surface enhanced Raman sample preparation $^{36}$

1. Add $980 \mu \mathrm{L}$ of prepared colloidal solution to $1 \mathrm{~mL}$ sample vile.

2. Add $10 \mu \mathrm{L}$ of aggregating agent to colloidal solution, cap and vortex for 30 seconds.

3. Add $10 \mu \mathrm{L}$ of sample to colloidal solution mixed with aggregating agent and vortex for 30 seconds.

4. Samples are allowed to react for 40 minutes prior to running Raman analysis. 
APPENDIX D: Standard operating procedure for supported liquid extraction ${ }^{73}$ Extraction using Isolute SLE $+2 \mathrm{~mL}$ extraction columns from Biotage.

1. Sample Load:
a. Load $2 \mathrm{~mL}$ of pretreated sample on top of column
b. Pulse vacuum to initiate flow
c. Leave for 5 minutes to completely adsorb

2. Sample Elution:
a. Add $2.5 \mathrm{~mL}$ of dichloromethane to top of column
b. Allow to flow under gravity for 5 minutes
c. Add additional $2.5 \mathrm{~mL}$ of dichloromethane to top of column
d. Allow to flow under gravity for 5 minutes
e. Apply short pulse vacuum to elute

3. Sample Evaporation:
a. Evaporate sample to dryness
b. Reconstitute in $100 \mu \mathrm{L}$ of $10 \%$ methanol 
VITA

ERIKA L. DOCTOR

Born, Johnston, Rhode Island

2003-2007

B.S., Chemistry

Cedar Crest College

Allentown, Pennsylvania

2007-2008

M.S., Forensic Science

Cedar Crest College

Allentown, Pennsylvania

2010-2014

Head Teaching Assistant

Florida International University

Miami Florida

2008-2014

Teaching Assistant

Florida International University

Miami Florida

2012-2014

Doctoral Candidate

Florida International University

Miami Florida

\section{PUBLICATIONS AND PRESENTATIONS}

Erika L. Doctor and Bruce McCord "Comparison of Aggregating Agents for the SurfaceEnhanced Raman Analysis of Benzodiazepine.” Analyst, 2013, 138 (20), 5926 - 5932

Maximilien Blas, Erika L. Asselin, Tao Liu, Cristina Rodriguez, Roberto R. Panepucci, and Bruce R. McCord "Minimizing the Number of Voltage Sources for Pinched Injection on a Microfluidic Device." Lab-on-a-Chip, 2010, 10, 1319-1323

Erika L. Doctor and Bruce McCord, "Development of a surface-enhanced Raman spectroscopy method for the detection of benzodiazepines in urine." 2013, Sumerset, NJ. Oral presentation, invited speaker, Eastern Analytical Symposium

Erika L. Doctor and Bruce McCord, "Development of a supported liquid extraction method for benzodiazepines in urine with surface-enhanced Raman spectroscopy detection." 2014, Seattle, WA. Poster presentation. American Academy of Forensic Sciences 
Erika L. Doctor and Bruce McCord, "Development of a surface-enhanced Raman spectroscopy method for the detection of benzodiazepines in urine." 2013, Washington D.C. Oral presentation. American Academy of Forensic Sciences

Erika L. Doctor and Bruce McCord, "Comparison of aggregating agents for surfaceenhanced Raman analysis of benzodiazepines." 2012, Atlanta, Georgia. Poster presentation. American Academy of Forensic Sciences

Erika L. Asselin and Bruce McCord, "Surface-enhanced Raman analysis of benzodiazepines using silver colloidal dispersions" 2010, Seattle, Washington. Poster presentation. American Academy of Forensic Sciences

Erika L. Asselin and Thomas Brettell, "Presumptive and confirmatory identification of 1,2-triazolo-benzodiazepines." 2008, Washington, D.C. Poster presentation. American Academy of Forensic Sciences

Erika L. Doctor and Bruce McCord, "Surface enhanced Raman analysis of benzodiazepines using gold colloidal dispersions.” 2011, Tampa, Florida. Oral Presentation. Florida Annual Meeting and Exposition

Erika L. Doctor and Bruce McCord, "Surface enhanced Raman analysis of benzodiazepines using gold colloidal dispersions." 2011, Miami, Florida. Poster presentation. NanoFlorida NanoScience Technology Symposium 